\title{
CHARACTERIZATION OF FUNCTION SPACES OF VECTOR FIELDS AND AN APPLICATION IN NONLINEAR PERIDYNAMICS
}

\author{
TADELE MENGESHA AND QIANG DU
}

\begin{abstract}
The paper introduces a class of nonlocal derivative operators defined over vector fields that turn out to satisfy a nonlocal integration by parts formula. We demonstrate that in several function spaces, these operators have similar continuity property as the classical differential operators. A closed formula for the limit of these operators will be obtained when nonlocality vanishes. The limit analysis together with the integration by parts formula enable us link the nonlocal derivative operators and associated function spaces with the conventional local differential operators and Sobolev, bounded variations, and bounded deformation function spaces. As an application, we present an existence result and asymptotic analysis in the sense of $\Gamma$-convergence of some nonlinear variational problems that arise in nonlocal continuum mechanics.
\end{abstract}

Key words and phrases: Nonlocal characterization, Peridynamics, $\Gamma$-convergence

\section{INTRODUCTION}

For a given $\Omega \subset \mathbb{R}^{d}$ that is open, bounded and sufficiently smooth, a measurable vector field $\mathbf{u}: \Omega \rightarrow \mathbb{R}^{d}$ and $\mathbf{x} \in \Omega$, we introduce and study the nonlocal gradient matrix defined as

$$
\mathbb{G}_{\rho}^{\mathrm{k}} \mathbf{u}(\mathbf{x}):=\lim _{\epsilon \rightarrow 0} \int_{\Omega \backslash B(\mathbf{x}, \epsilon)} \rho(\mathbf{y}-\mathbf{x}) \mathrm{K}(\mathbf{y}-\mathbf{x}) \mathcal{D}_{*}(\mathbf{u})(\mathbf{y}, \mathbf{x}) d \mathbf{y} .
$$

The operator $\mathbb{G}_{\rho}^{k}$ acts on $\mathbf{u}$ through the difference quotient $\mathcal{D}_{*}(\mathbf{u})(\mathbf{x}, \mathbf{y})=\frac{\mathbf{u}(\mathbf{x})-\mathbf{u}(\mathbf{y})}{|\mathbf{x}-\mathbf{y}|}$. The nonnegative kernel $\rho=\rho(|\boldsymbol{\xi}|)$ defined in $\mathbb{R}^{d}$ is assumed to be radial and locally integrable. The field $\boldsymbol{\xi} \mapsto \mathrm{K}(\boldsymbol{\xi})$ is a third order tensor field with entries $\mathrm{K}_{i j m}$ and the property that,

$$
\mathrm{K}(-\boldsymbol{\xi})=-\mathrm{K}(\boldsymbol{\xi}), \quad \mathrm{K}(r \boldsymbol{\xi})=\mathrm{K}(\boldsymbol{\xi}), \forall r>0, \text { and }\left\|\mathrm{K}_{i j m}\right\|_{L^{\infty}} \leq C, \forall i, j, m \in\{1,2, \cdots, d\}
$$

for some universal positive constant $C$. The product $\mathrm{K}(\mathbf{y}-\mathbf{x}) \mathcal{D}_{*}(\mathbf{u})(\mathbf{y}, \mathbf{x})$ is a matrix and is given by the standard multiplication via contraction of tensors that will be explained shortly. As such $\mathbb{G}_{\rho}^{k} \mathbf{u}(\mathbf{x})$ is by definition a matrixvalued weighted difference quotient of the vector field $\mathbf{u}$. We call $\mathbb{G}_{\rho}^{k} \mathbf{u}(\mathbf{x})$ a nonlocal gradient matrix at $\mathbf{x}$ of $\mathbf{u}$ and the operator $\mathbb{G}_{\rho}^{k}$ the nonlocal gradient matrix operator. Our aim in this paper is to study several aspects of the operator $\mathbb{G}_{\rho}^{\mathrm{k}}$. We plan to study the continuity properties of $\mathbb{G}_{\rho}^{k}$ in several topologies. We will be able to define a dual operator of $\mathbb{G}_{\rho}^{\mathrm{k}}$ that will lead to a nonlocal integration by parts formula. In the event that the kernel $\rho$ approximates the Dirac Delta-function, we establish connections between $\mathbb{G}^{k} \rho$ and some differential operators a consequence of which is a nonlocal characterization of Sobolev and function spaces.

Before we start describing our result, we would like to mention that this work is motivated by two recent developments. The first one is the introduction of the peridynamics model (PD) of continuum mechanics [33, 34, 35]. The PD model uses integral operators having close resemblance with (1.1) to describe physical and mechanical quantities and laws. For calibration purposes it is required that, in the event of vanishing nonlocality, the nonlocal models 'converge' to a classical local PDE model when the latter is physically valid and mathematically well-posed. Indeed, various PD models and their solutions are shown to converge to local counterparts, see $[19,27,26,17,14,24]$. Such type of compatibility between the local and nonlocal models has drawn some studies on the 'correspondence formulation' of peridynamics which conveniently replaces classical differential operators by corresponding nonlocal terms to derive constitutive relations, though it has been noted that such a practice should be subject to much scrutiny, see [35, 38, 39]. Meanwhile, the introduction of PD has also motivated the nonlocal vector calculus developed by $\mathrm{Du}$ et al. in $[18,17]$ that provides a framework to analyze nonlocal operators, their connections via identities such as the nonlocal integration by parts formula, and their relationship 
to corresponding classical and local differential operators. A rigorous justification of the localization of nonlocal gradients to the classical one in several topologies is provided by [29] for scalar fields defined on a bounded domain. These earlier works have largely focused on the special case of low-rank tensors while our study here extends the result in $[18,17,29]$ to vector fields and high-rank tensors as given by $(1.2)$. In addition, our work is also inspired by the work of Bourgain, Brezis, Mironescu [9], Brezis [11], Ponce [30, 31] and Davila [16] on nonlocal characterization of Sobolev and BV function spaces that have been used in the analysis of image processing [6] and nonlocal diffusion models [4]. Following the approach in [9] pedantically, albeit in a nontransparent way, a nonlocal characterization of the space of $B D$ functions is also provided in [25]. In this paper, we will present a unified approach to characterize $W^{1, p}, B V, B D, H(\operatorname{div})$ and other function spaces via generalized nonlocal means. The new approach not only makes the proofs of nonlocal characterizations simpler, but also allows the use of weaker conditions to obtain the desired results. We should mention that a characterization of higher order Sobolev spaces in the spirit of Bourgain, Brezis, Mironescu can be found in [8, 7, 37].

Let us summarize our findings. The boundedness of the nonlocal gradient for vector fields (1.1) on the space of Sobolev, bounded variation (BV) and bounded deformation (BD) spaces of vector fields will be demonstrated. A corresponding adjoint nonlocal divergent operator is defined and a nonlocal integration by parts formula will be proved. In the event that the kernel $\rho$ behaves like an approximation of the Dirac-Delta function, the nonlocal gradient operators will be shown to be close to classical (and local) gradient operators in several topologies. This localization together with the nonlocal integration by parts formula will be used to characterize classical Sobolev, $\mathrm{BV}$ and $\mathrm{BD}$ vector fields via nonlocal means, in the same spirit of the nonlocal characterization of Sobolev spaces of scalar-valued functions given by Bourgain Brezis, and Mironescu [9], Brezis [11], Ponce [30, 31], and Davila [16]. Again, the extension to spaces of vector fields represents a major new contribution of the current work. In the context of peridynamics, for some special third order fields to be explicitly stated later, we note that $\mathbb{G}_{\rho}^{\mathrm{k}} \mathbf{u}(\mathbf{x})$ will be realized as a weighted average of the so-called nonlocal volumetric strain $\mathscr{D}(\mathbf{u})(\mathbf{x}, \mathbf{y})=\mathcal{D}_{*}(\mathbf{u})(\mathbf{x}, \mathbf{y}) \cdot \frac{(\mathbf{x}-\mathbf{y})}{|\mathbf{x}-\mathbf{y}|}$ as defined in [34] for a displacement field $\mathbf{u}$. As an application, we provide some new results on the mathematical analysis of a nonlinear nonlocal variational problems in small strain theory that uses the nonlocal volumetric strain $\mathscr{D}(\mathbf{u})(\mathbf{x}, \mathbf{y})$. The focus is on minimizing a nonlocal strain energy which is decomposed as a sum of a deviatoric strain energy and the dilatation strain energy. In the event of vanishing nonlocality, the $\Gamma$-convergence of the nonlocal energy functional on a class of function spaces will be demonstrated. We remark that variational convergence of nonlocal energy functionals to local energies for peridynamic models has also been performed recently. In [23, 24], $\Gamma$ - limit techniques are applied to nonlinear peridynamic evolutions. These dynamic problems involve unstable peridynamics constitutive laws leading to a variational limit dynamics that captures the simultaneous evolution of linear elastic displacement and a fracture set across which the displacement is discontinuous. In [13, 14], similar to our work but distinct existence and $\Gamma$-convergence techniques are applied to study the limiting behavior of large deformation peridynamic models towards deformation gradient-based potentials that can be obtained as quasi-convexfication of pointwise limits (when horizon diminishes) of nonlocal integrands. While our nonlocal energy functional is a function of the nonlocal volumetric strain $\mathscr{D}(\mathbf{u})(\mathbf{x}, \mathbf{y})=\mathcal{D}_{*}(\mathbf{u})(\mathbf{x}, \mathbf{y}) \cdot \frac{(\mathbf{x}-\mathbf{y})}{|\mathbf{x}-\mathbf{y}|}$, reflecting the fact that we are working on small strain theory, the functionals studied in $[13,14]$ use the full difference quotient $\mathcal{D}_{*}(\mathbf{u})(\mathbf{x}, \mathbf{y})$. Moreover, in this work we use a possible distinct growth conditions at infinity for the two components of the nonlocal strain energy, as opposed to the same growth required in our recent work [28].

Let us now give some special examples of third order tensor fields that satisfy (1.2) and that we will explore further in later sections. These special tensors, sometime called shape tensors in the context of peridynamics, are given as follows:

$$
\boldsymbol{\xi} \mapsto \frac{\boldsymbol{\xi}}{|\boldsymbol{\xi}|} \otimes \mathbb{I} ; \quad \boldsymbol{\xi} \mapsto \frac{\boldsymbol{\xi}}{|\boldsymbol{\xi}|} \otimes \frac{\boldsymbol{\xi}}{|\boldsymbol{\xi}|} \otimes \frac{\boldsymbol{\xi}}{|\boldsymbol{\xi}|} ; \quad \boldsymbol{\xi} \mapsto \mathbb{I} \otimes \frac{\boldsymbol{\xi}}{|\boldsymbol{\xi}|} ; \quad \boldsymbol{\xi} \mapsto\left(\frac{\boldsymbol{\xi}}{|\boldsymbol{\xi}|} \otimes \frac{\boldsymbol{\xi}}{|\boldsymbol{\xi}|}-\gamma \mathbb{I}\right) \otimes \frac{\boldsymbol{\xi}}{|\boldsymbol{\xi}|}, \gamma>0 .
$$

Hereafter the product $\otimes$ is defined by the standard convention, that is, for any pair of vectors $\mathbf{v}$ and $\mathbf{w}$, and any matrix $\mathbb{A}$, we have $(\mathbf{v} \otimes \mathbf{w})_{i j}=\mathbf{v}_{i} \mathbf{w}_{j}$ and $(\mathbb{A} \otimes \mathbf{v})_{i j k}=(\mathbb{A})_{i j} \mathbf{v}_{k}$. We also define two actions of a third order tensor $\mathrm{K}$ on a vector $\mathbf{v}, \mathrm{K} \mathbf{v}$ and $\mathrm{K} \odot \mathbf{v}$. These products are matrices defined as

$$
[\mathrm{K} \mathbf{v}]_{i j}=\sum_{k} \mathrm{~K}_{i j k} \mathbf{v}_{k}, \quad[\mathrm{~K} \odot \mathbf{v}]_{i j}=\sum_{k} \mathrm{~K}_{i k j} \mathbf{v}_{k} .
$$

From the definitions above it is not hard to see that for any vectors $\mathbf{v}, \mathbf{w}$, a matrix $\mathbb{A}$ and a third order tensor $\mathrm{K}$,

$$
[\mathrm{K} \mathbf{v}] \mathbf{w}=(\mathrm{K} \odot \mathbf{w}) \mathbf{v}, \quad[(\mathbb{A} \otimes \mathbf{w})] \mathbf{v}=(\mathbf{w} \cdot \mathbf{v}) \mathbb{A} .
$$


In addition, $\mathrm{K}[\mathbb{A} \mathbf{v}]$ determines a matrix through the usual contraction multiplication of tensors as given by

$$
[\mathrm{K}[\mathbb{A} \mathbf{v}]]_{i j}=\sum_{k, l} \mathrm{~K}_{i j k} \mathbb{A}_{k l} \mathbf{v}_{l}
$$

which is essentially the standard tensor-matrix-vector multiplication. The generic notation $|\cdot|$ may denote the absolute value, the norm of a vector or the standard matrix norm. From the inequality in (1.2) we note that there exists a universal constant $C$ such that for any vectors $\mathbf{v}, \mathbf{w}$

$$
\sup _{|\boldsymbol{\xi}| \leq 1}|\mathrm{~K}(\boldsymbol{\xi}) \mathbf{v}| \leq C|\mathbf{v}|, \quad \sup _{|\boldsymbol{\xi}| \leq 1}|\mathrm{~K}(\boldsymbol{\xi}) \odot \mathbf{w}| \leq C|\mathbf{w}|
$$

The rest of the paper is organized as follows: first, we present the properties of the nonlocal gradient operator and the associated nonlocal divergence in Section 2, along with the establishment of the nonlocal integration by parts formula. The localizations are considered in Section 3. Different interpretations of the nonlocal operators are provided along with discussions of such operators associated with specially defined kernels. Nonlocal characterizations of spaces are also established in Section 3. Applications to some nonlinear nonlocal variational problems are considered in Section 4. Some conclusions and discussion of open issues are given in Section 5.

\section{NONLOCAL OPERATORS}

In this section, we study various properties of the nonlocal gradient operator $\mathbb{G}_{\rho}^{\mathrm{k}}$ and its dual operator. Unless it is explicitly stated, we assume that $\mathrm{K}$ is a generic third order tensor field satisfying (1.2). A nonlocal integration by parts formula is also rigorously established here. For notation simplicity, throughout the paper we use $c$ and $C$ to denote generic positive constants that are only model dependent. We denote a ball centered at a and radius $r$ by $B_{r}(\mathbf{a})$ or $B(\mathbf{a}, r)$, when appropriate. The definition of the Sobolev space is standard, and the space $B V\left(\Omega ; \mathbb{R}^{d}\right)$ is collection of vector fields $\mathbf{u} \in L^{1}\left(\Omega ; \mathbb{R}^{d}\right)$ whose distributional gradient matrix $D \mathbf{u}$ is a finite Radon measure in $\Omega$. $B V\left(\Omega ; \mathbb{R}^{d}\right)$ is a Banach space equipped with the norm $\|\mathbf{u}\|_{B V}:=\|\mathbf{u}\|_{L^{1}}+\|D u\|(\Omega)$, where the total variation $\|D u\|(\Omega)$ is given by

$$
\|D u\|(\Omega)=\sup \left\{\int_{\Omega} \mathbf{u} \cdot \operatorname{div} \mathbf{\Phi} d \mathbf{x}: \mathbf{\Phi} \in C_{c}^{1}\left(\Omega ; \mathbb{R}^{d \times d}\right)\right\} .
$$

The space $B D(\Omega)$ is the set of vector fields $\mathbf{u} \in L^{1}\left(\Omega ; \mathbb{R}^{d}\right)$ whose distributional symmetric gradient matrix $\mathcal{E}(\mathbf{u})$ is a finite Radon measure in $\Omega$. By definition, the $i j$ entry of $\mathcal{E}(\mathbf{u})$ is given by

$$
\left\langle\mathcal{E}_{i j}(\mathbf{u}), \psi\right\rangle:=-\frac{1}{2} \int_{\Omega}\left(u_{j} \frac{\partial \psi}{\partial x_{i}}+u_{i} \frac{\partial \psi}{\partial x_{j}}\right) d \mathbf{x}, \quad \text { for all } \psi \in C_{c}^{1}(\Omega) .
$$

Again, $B D(\Omega)$ is a Banach space equipped with the norm $\|\mathbf{u}\|_{B D}:=\|\mathbf{u}\|_{L^{1}}+\|\mathcal{E}(\mathbf{u})\|(\Omega)$, where $\|\mathcal{E}(\mathbf{u})\|(\Omega)$ is the total variation of the measure $\mathcal{E}(\mathbf{u})$ is defined as (equivalently) $\|\mathcal{E}(\mathbf{u})\|(\Omega)=\sum_{i j}\left|\mathcal{E}_{i j}\right|(\Omega)$, and

$$
\left|\mathcal{E}_{i j}\right|(\Omega)=\sup \left\{\left\langle\mathcal{E}_{i j}(\mathbf{u}), \psi\right\rangle: \psi \in C_{c}^{1}(\Omega)\right\} .
$$

The nonlocal gradient $\mathbb{G}_{\rho}^{\mathrm{k}}$. Let us first establish some continuity properties of the nonlocal gradient operator $\mathbb{G}_{\rho}^{\mathrm{k}}$ on several function spaces.

\section{Proposition 2.1.}

(1) The map $\mathbb{G}_{\rho}^{\mathrm{k}}: C^{1}\left(\bar{\Omega} ; \mathbb{R}^{d}\right) \rightarrow C\left(\Omega ; \mathbb{R}^{d \times d}\right)$ is a bounded linear operator with the estimate

$$
\left.\left\|\mathbb{G}_{\rho}^{\mathrm{k}} \mathbf{u}\right\|_{L^{\infty}(\Omega)} \leq C\|\rho\|_{L^{1}}\|\nabla \mathbf{u}\|_{L^{\infty}} \quad \forall \mathbf{u} \in C^{1}\left(\bar{\Omega} ; \mathbb{R}^{d}\right)\right),
$$

for some positive constant $C$ depending only on $\Omega$.

(2) For $1 \leq p \leq \infty$, the map $\mathbb{G}_{\rho}^{\mathrm{k}}: W^{1, p}\left(\Omega ; \mathbb{R}^{d}\right) \rightarrow L^{p}\left(\Omega ; \mathbb{R}^{d \times d}\right)$ is a bounded linear operator with the estimate that

$$
\left\|\mathbb{G}_{\rho}^{\mathrm{k}} \mathbf{u}\right\|_{L^{p}\left(\Omega ; \mathbb{R}^{d \times d}\right)} \leq C\|\rho\|_{L^{1}}\|\nabla \mathbf{u}\|_{L^{p}\left(\Omega ; \mathbb{R}^{d \times d}\right)}, \quad \forall \mathbf{u} \in W^{1, p}\left(\Omega ; \mathbb{R}^{d}\right),
$$

for some positive constant $C$ depending only on $p$ and $\Omega$.

(3) The map $\mathbb{G}_{\rho}^{k}: B V\left(\Omega ; \mathbb{R}^{d}\right) \rightarrow L^{1}\left(\Omega ; \mathbb{R}^{d \times d}\right)$ is a bounded linear operator with the estimate that

$$
\left\|\mathbb{G}_{\rho}^{\mathrm{k}} \mathbf{u}\right\|_{L^{1}} \leq C\|\rho\|_{L^{1}}\|D \mathbf{u}\|(\Omega), \quad \forall \mathbf{u} \in B V\left(\Omega ; \mathbb{R}^{d}\right)
$$

for some positive constant $C$ depending only on $\Omega$. 
(4) When $\mathrm{K}(\boldsymbol{\xi})=\frac{\boldsymbol{\xi}}{|\boldsymbol{\xi}|} \otimes \frac{\boldsymbol{\xi}}{|\boldsymbol{\xi}|} \otimes \frac{\boldsymbol{\xi}}{|\boldsymbol{\xi}|}$ or $\mathrm{K}(\boldsymbol{\xi})=\mathbb{I} \otimes \frac{\boldsymbol{\xi}}{|\boldsymbol{\xi}|}$, then $\mathbb{G}_{\rho}^{\mathrm{k}}: B D\left(\Omega ; \mathbb{R}^{d}\right) \rightarrow L^{1}\left(\Omega ; \mathbb{R}^{d \times d}\right)$ is a bounded linear operator with the estimate that

$$
\left\|\mathbb{G}_{\rho}^{\mathrm{k}} \mathbf{u}\right\|_{L^{1}} \leq C\|\rho\|_{L^{1}}\|\mathbf{u}\|_{B D}, \quad \mathbf{u} \in B D\left(\Omega ; \mathbb{R}^{d}\right)
$$

for some positive constant $C$ depending only on $\Omega$.

In all the above, by abuse of notation, we take $\|\rho\|_{L^{1}}$ to mean the $L_{l o c}^{1}$ norm over a bounded set.

Proof. We first prove part 1. For any $\mathbf{x}, \mathbf{y} \in \Omega$, and $\mathbf{u} \in C^{1}\left(\bar{\Omega} ; \mathbb{R}^{d}\right)$ using mean value theorem, there exists $\boldsymbol{\xi}(\mathbf{y}, \mathbf{x}) \in \bar{\Omega}$ such that

$$
\mathcal{D}_{*}(\mathbf{u})(\mathbf{y}, \mathbf{x})=\nabla \mathbf{u}(\boldsymbol{\xi}(\mathbf{y}, \mathbf{x})) \frac{\mathbf{y}-\mathbf{x}}{|\mathbf{y}-\mathbf{x}|}
$$

and as a consequence we have

$$
\rho(\mathbf{y}-\mathbf{x}) \mathrm{K}(\mathbf{y}-\mathbf{x}) \mathcal{D}_{*}(\mathbf{u})(\mathbf{y}, \mathbf{x})=\rho(\mathbf{y}-\mathbf{x}) \mathrm{K}(\mathbf{y}-\mathbf{x}) \nabla \mathbf{u}(\boldsymbol{\xi}(\mathbf{y}, \mathbf{x})) \frac{\mathbf{y}-\mathbf{x}}{|\mathbf{y}-\mathbf{x}|} .
$$

Then it is clear that for all $\mathbf{x} \in \Omega, \mathbb{G}_{\rho}^{k} \mathbf{u}(\mathbf{x})$ is well defined and coincides with the Lebesgue integral

$$
\mathbb{G}_{\rho}^{\mathrm{k}} \mathbf{u}(\mathbf{x})=\int_{\Omega} \rho(\mathbf{y}-\mathbf{x}) \mathrm{K}(\mathbf{y}-\mathbf{x}) \mathcal{D}_{*}(\mathbf{u})(\mathbf{y}, \mathbf{x}) d \mathbf{y} .
$$

Moreover, using (1.4), we have $\mathbb{G}_{\rho}^{\mathrm{k}} \mathbf{u} \in L^{\infty}\left(\Omega ; \mathbb{R}^{d \times d}\right)$ with the pointwise estimate that

$$
\left|\mathbb{G}_{\rho}^{\mathrm{k}} \mathbf{u}(\mathbf{x})\right| \leq C \int_{\Omega} \rho(\mathbf{y}-\mathbf{x}) d \mathbf{x}\|\nabla \mathbf{u}\|_{L^{\infty}} \leq C\|\rho\|_{L^{1}}\|\nabla \mathbf{u}\|_{L^{\infty}}
$$

We also observe that as $\epsilon \rightarrow 0$,

$$
\left|\int_{\Omega \backslash B_{\epsilon}(\mathbf{x})} \rho(\mathbf{y}-\mathbf{x}) \mathrm{K}(\mathbf{y}-\mathbf{x}) \mathcal{D}_{*}(\mathbf{u})(\mathbf{y}, \mathbf{x}) d \mathbf{y}-\mathbb{G}_{\rho}^{\mathrm{k}} \mathbf{u}(\mathbf{x})\right| \leq C\|\nabla \mathbf{u}\|_{L^{\infty}} \int_{B_{\epsilon}(\mathbf{0})} \rho(\mathbf{z}) d \mathbf{z} \rightarrow 0
$$

uniformly in $\Omega$. Thus to show that $\mathbb{G}_{\rho}^{\mathrm{k}} \mathbf{u}$ is continuous, it suffices to show that $\mathbb{G}_{\rho_{\epsilon}}^{\mathrm{k}} \mathbf{u}$ is continuous in $\Omega$ for each $\epsilon>0$ where $\rho_{\epsilon}(|\mathbf{z}|)=\rho(|\mathbf{z}|) \chi_{[\epsilon, \infty)}(|\mathbf{z}|)$. We note that the continuity of $\mathbb{G}_{\rho_{\epsilon}}^{\mathrm{k}} \mathbf{u}$ follows from the fact that it can be written as

$$
\mathbb{G}_{\rho_{\epsilon}}^{\mathrm{k}} \mathbf{u}(\mathbf{x})=\int_{\Omega} \rho_{\epsilon}(\mathbf{y}-\mathbf{x}) \mathrm{K}(\mathbf{y}-\mathbf{x}) \frac{\mathbf{u}(\mathbf{y})}{|\mathbf{y}-\mathbf{x}|} d \mathbf{y}-\mathrm{A}_{\epsilon}(\mathbf{x}) \mathbf{u}(\mathbf{x}), \quad A_{\epsilon}(\mathbf{x})=\int_{\Omega} \rho_{\epsilon}(\mathbf{y}-\mathbf{x}) \frac{\mathrm{K}(\mathbf{y}-\mathbf{x})}{|\mathbf{y}-\mathbf{x}|} d \mathbf{y}
$$

with each one of the two terms in the first equation above being continuous functions individually.

Now for part 2 , the case $p=\infty$ can be shown in the same way as above. Let us show the boundedness of $\mathbb{G}^{\mathrm{k}} \rho$ as a map from $W^{1, p}\left(\Omega ; \mathbb{R}^{d}\right)$ to $L^{p}\left(\Omega ; \mathbb{R}^{d \times d}\right)$ when $1 \leq p<\infty$. To that end, we recall the estimate

$$
\left(\iint_{\Omega \times \Omega} \rho(\mathbf{y}-\mathbf{x})\left|\mathcal{D}_{*} \mathbf{u}(\mathbf{y}, \mathbf{x})\right|^{p} d \mathbf{y} d \mathbf{x}\right)^{1 / p} \leq C\|\rho\|_{L^{1}}\|\nabla \mathbf{u}\|_{L^{p}}
$$

that holds for any $\rho \in L_{l o c}^{1}$ and $\mathbf{u} \in W^{1, p}\left(\Omega ; \mathbb{R}^{d \times d}\right)$ as shown in [9, Theorem 1]. Applying Hölder's inequality first and then Minkowski's inequality, we obtain

$$
\begin{aligned}
& \iint_{\Omega \times \Omega}\left|\rho(\mathbf{y}-\mathbf{x}) \mathrm{K}(\mathbf{y}-\mathbf{x}) \mathcal{D}_{*} \mathbf{u}(\mathbf{y}, \mathbf{x})\right| d \mathbf{y} d \mathbf{x} \leq C\left(\int_{\Omega}\left|\int_{\Omega}\right| \rho(\mathbf{y}-\mathbf{x}) \mathrm{K}(\mathbf{y}-\mathbf{x}) \mathcal{D}_{*}(\mathbf{u})(\mathbf{y}, \mathbf{x})|d \mathbf{y}|^{p} d \mathbf{x}\right)^{1 / p} \\
& \leq C\|\rho\|_{L^{1}}\left(\iint_{\Omega \times \Omega} \rho(\mathbf{y}-\mathbf{x})\left|\mathcal{D}_{*} \mathbf{u}(\mathbf{y}, \mathbf{x})\right|^{p} d \mathbf{y} d \mathbf{x}\right)^{1 / p} \leq C\|\rho\|_{L^{1}}\|\nabla \mathbf{u}\|_{L^{p}} .
\end{aligned}
$$

This implies that by Fubini's theorem for almost all $\mathbf{x} \in \Omega$, the function

$$
\mathbf{y} \rightarrow \rho(\mathbf{y}-\mathbf{x}) \mathrm{K}(\mathbf{y}-\mathbf{x}) \mathcal{D}_{*} \mathbf{u}(\mathbf{y}, \mathbf{x})
$$

is in $L^{1}\left(\Omega ; \mathbb{R}^{d \times d}\right)$ and therefore using it as a majorizing function, and applying dominated convergence theorem, we obtain that as $\epsilon \rightarrow 0$,

$$
\mathbb{G}_{\rho}^{\mathrm{k}} \mathbf{u}(\mathbf{x})=\lim _{\epsilon \rightarrow 0} \mathbb{G}_{\rho_{\epsilon}}^{\mathrm{k}} \mathbf{u}(\mathbf{x})=\int_{\Omega} \rho(\mathbf{y}-\mathbf{x}) \mathrm{K}(\mathbf{y}-\mathbf{x}) \mathcal{D}_{*} \mathbf{u}(\mathbf{y}, \mathbf{x}) \mid d \mathbf{y}
$$


strongly in $L^{1}\left(\Omega ; \mathbb{R}^{d \times d}\right)$. Also as in the above,

$$
\left\|\mathbb{G}_{\rho}^{\mathrm{k}} \mathbf{u}\right\|_{L^{p}} \leq C\|\rho\|_{L^{1}}\left(\iint_{\Omega \times \Omega} \rho(\mathbf{y}-\mathbf{x})\left|\mathcal{D}_{*} \mathbf{u}(\mathbf{y}, \mathbf{x})\right|^{p} d \mathbf{y} d \mathbf{x}\right)^{1 / p} \leq C\|\rho\|_{L^{1}}\|\nabla \mathbf{u}\|_{L^{p}},
$$

proving the boundedness from $W^{1, p}\left(\Omega ; \mathbb{R}^{d}\right)$ to $L^{p}\left(\Omega ; \mathbb{R}^{d \times d}\right)$.

As for parts 3 and 4 , we observe that the estimates for $\mathbb{G}_{\rho}^{k}$ when $\mathbf{u} \in B V(\Omega)$ and $\mathbf{u} \in B D(\Omega)$ follow from a similar argument as above and the inequalities that

$$
\iint_{\Omega \times \Omega} \rho(\mathbf{y}-\mathbf{x})\left|\mathcal{D}_{*} \mathbf{u}(\mathbf{y}, \mathbf{x})\right| d \mathbf{y} d \mathbf{x} \leq C\|\rho\|_{L^{1}}\|D \mathbf{u}\|(\Omega), \quad \forall \mathbf{u} \in B V\left(\Omega ; \mathbb{R}^{d}\right)
$$

as shown in $[9,11,16,31]$, and

$$
\iint_{\Omega \times \Omega} \rho(\mathbf{y}-\mathbf{x})\left|\frac{\mathbf{y}-\mathbf{x}}{|\mathbf{y}-\mathbf{x}|} \cdot \mathcal{D}_{*} \mathbf{u}(\mathbf{y}, \mathbf{x})\right| d \mathbf{y} d \mathbf{x} \leq C\|\rho\|_{L^{1}}\|\mathbf{u}\|_{B D}, \quad \forall \mathbf{u} \in B D(\Omega) .
$$

as shown in [25, Theorem 4.7].

Nonlocal divergence operator. Classical, local gradient operators and divergence operators form natural duality pairing that is viewed as an important property to be preserved in the development nonlocal vector calculus [18]. The duality pairing leads to a nonlocal analog of the classical integration by parts formula that plays important roles in variational analysis. Corresponding to operators given in (1.1), defined over the bounded domain $\Omega$, we formally define a nonlocal divergence operator as follows.

Definition 2.2. We define the operator $\mathbb{D}_{\rho}^{+}$as follows: for any measurable vector function $\phi: \Omega \rightarrow \mathbb{R}^{d}$, the function $\mathbb{D}_{\rho}^{+} \phi$ is given by

$$
\mathbb{D}_{\rho}^{+}(\phi)(\mathbf{x}):=-\lim _{\epsilon \rightarrow 0} \int_{\Omega \backslash B(\mathbf{x}, \epsilon)} \rho(\mathbf{y}-\mathbf{x}) \mathrm{K}(\mathbf{y}-\mathbf{x}) \odot \frac{\phi(\mathbf{y})+\phi(\mathbf{x})}{|\mathbf{y}-\mathbf{x}|} d \mathbf{y}, \quad \forall \mathbf{x} \in \Omega .
$$

In the above definition of $\mathbb{D}_{\rho}^{+}(\phi)$, it may appear that the validity of the integrals might be in question due to the lack of apparent cancellation, but we note that if $\delta(\mathbf{x})=\operatorname{dist}(\mathbf{x}, \partial \Omega)$ denotes the distance of a point $\mathbf{x} \in \Omega$ from the boundary, then

$$
\mathbb{D}_{\rho}^{+}(\phi)(\mathbf{x})=\mathbb{D}_{\rho}^{-}(\phi)(\mathbf{x})-2 \int_{\Omega} \chi_{[\delta(\mathbf{x}), \infty)}(|\mathbf{y}-\mathbf{x}|) \rho(\mathbf{y}-\mathbf{x}) \mathrm{K}(\mathbf{y}-\mathbf{x}) \odot \frac{\phi(\mathbf{x})}{|\mathbf{y}-\mathbf{x}|} d \mathbf{y}
$$

where

$$
\mathbb{D}_{\rho}^{-}(\phi)(\mathbf{x}):=-\lim _{\epsilon \rightarrow 0} \int_{\Omega \backslash B(\mathbf{x}, \epsilon)} \rho(\mathbf{y}-\mathbf{x}) \mathrm{K}(\mathbf{y}-\mathbf{x}) \odot \frac{\phi(\mathbf{y})-\phi(\mathbf{x})}{|\mathbf{y}-\mathbf{x}|} d \mathbf{y} .
$$

To demonstrate the validity of (2.1) we first observe that for $\mathbf{x} \in \Omega$ with $\delta(\mathbf{x})>0$ and for any $\epsilon \in(0, \delta(\mathbf{x}))$,

$$
\begin{aligned}
& -\chi_{[\epsilon, \infty)}(|\mathbf{x}-\mathbf{y}|) \rho(\mathbf{y}-\mathbf{x}) \mathrm{K}(\mathbf{y}-\mathbf{x}) \odot \frac{\phi(\mathbf{x})+\boldsymbol{\phi}(\mathbf{y})}{|\mathbf{x}-\mathbf{y}|}+2 \rho(\mathbf{y}-\mathbf{x}) \mathrm{K}(\mathbf{y}-\mathbf{x}) \odot \frac{\phi(\mathbf{x})}{|\mathbf{y}-\mathbf{x}|} \chi_{[\epsilon, \delta(\mathbf{x}))}(|\mathbf{x}-\mathbf{y}|) \\
& =-\chi_{[\epsilon, \infty)}(|\mathbf{x}-\mathbf{y}|) \rho(\mathbf{y}-\mathbf{x}) \mathrm{K}(\mathbf{y}-\mathbf{x}) \odot \frac{\phi(\mathbf{y})-\boldsymbol{\phi}(\mathbf{x})}{|\mathbf{x}-\mathbf{y}|}-2 \rho(\mathbf{y}-\mathbf{x}) \mathrm{K}(\mathbf{y}-\mathbf{x}) \odot \frac{\phi(\mathbf{x})}{|\mathbf{y}-\mathbf{x}|} \chi_{[\delta(\mathbf{x}), \infty)}(|\mathbf{x}-\mathbf{y}|) .
\end{aligned}
$$

Integrating both sides with respect to $\mathbf{y}$ we obtain

$$
\begin{aligned}
\int_{\Omega \backslash B(\mathbf{x}, \epsilon)} & \rho(\mathbf{y}-\mathbf{x}) \mathrm{K}(\mathbf{y}-\mathbf{x}) \odot \frac{\phi(\mathbf{x})+\phi(\mathbf{y})}{|\mathbf{x}-\mathbf{y}|} d \mathbf{y}=\int_{\Omega \backslash B(\mathbf{x}, \epsilon)} \rho(\mathbf{y}-\mathbf{x}) \mathrm{K}(\mathbf{y}-\mathbf{x}) \odot \frac{\phi(\mathbf{y})-\phi(\mathbf{x})}{|\mathbf{x}-\mathbf{y}|} d \mathbf{y} \\
& +2 \int_{\Omega} \rho(\mathbf{y}-\mathbf{x}) \mathrm{K}(\mathbf{y}-\mathbf{x}) \frac{\phi(\mathbf{x})}{|\mathbf{y}-\mathbf{x}|} \chi_{[\delta(\mathbf{x}), \infty)}(|\mathbf{x}-\mathbf{y}|) d \mathbf{y},
\end{aligned}
$$

where we have used the fact that since $\mathrm{K}$ is an odd third order tensor field

$$
\int_{\Omega} 2 \rho(\mathbf{y}-\mathbf{x}) \mathrm{K}(\mathbf{y}-\mathbf{x}) \odot \frac{\phi(\mathbf{x})}{|\mathbf{y}-\mathbf{x}|} \chi_{[\epsilon, \delta(\mathbf{x}))}(|\mathbf{x}-\mathbf{y}|) d \mathbf{y}=2 \int_{B_{\delta(\mathbf{x})}(\mathbf{0}) \backslash B_{\epsilon}(\mathbf{0})} \rho(\mathbf{z}) \mathrm{K}(\mathbf{z}) \odot \frac{\phi(\mathbf{x})}{|\mathbf{z}|} d \mathbf{z}=0 .
$$

Equation (2.1) now follows by taking the limit as $\epsilon \rightarrow 0$ in (2.3).

The following continuity property of the operators $\mathbb{D}_{\rho}^{+}$and $\mathbb{D}_{\rho}^{-}$will be useful throughout the paper. 
Lemma 2.3. Given $\phi \in C_{c}^{1}\left(\Omega ; \mathbb{R}^{d}\right)$, we have $\mathbb{D}_{\rho}^{+}(\phi)$ and $\mathbb{D}_{\rho}^{-}(\phi) \in L^{\infty}\left(\Omega ; \mathbb{R}^{d}\right)$, with the pointwise estimate

$$
\left|\mathbb{D}_{\rho}^{-}(\boldsymbol{\phi})(\mathbf{x})\right| \leq C\|\rho\|_{L^{1}}\|\nabla \phi\|_{L^{\infty}}, \quad\left|\mathbb{D}_{\rho}^{+}(\boldsymbol{\phi})(\mathbf{x})\right| \leq C\|\rho\|_{L^{1}}\|\nabla \phi\|_{L^{\infty}}
$$

for all $\mathbf{x} \in \Omega$, and a universal positive constant $C$.

Proof. The bound on $\left|\mathbb{D}_{\rho}^{-}(\phi)(\mathbf{x})\right|$ can be obtained similarly as in the proof of the Proposition 2.1 using the assumption (1.4). We will use the relation (2.1) to prove the bound on $\left|\mathbb{D}_{\rho}^{+}(\phi)(\mathbf{x})\right|$ and it suffices to estimate the second term in the right hand side of (2.1). To that end, corresponding to $\mathbf{x} \in \Omega$, choose $\xi(\mathbf{x}) \in[\Omega \backslash \operatorname{supp}(\phi)] \cap$ $B(\mathbf{x}, \delta(\mathbf{x}))$. Then $\phi(\xi(\mathbf{x}))=0$ and so

$$
\begin{aligned}
& \int_{\Omega} \chi_{[\delta(\mathbf{x}), \infty)}(|\mathbf{x}-\mathbf{y}|) \rho(\mathbf{y}-\mathbf{x}) \mathrm{K}(\mathbf{y}-\mathbf{x}) \odot \frac{\phi(\mathbf{x})}{|\mathbf{y}-\mathbf{x}|} d \mathbf{y} \\
& \quad=\int_{\Omega} \chi_{[\delta(\mathbf{x}), \infty)}(|\mathbf{x}-\mathbf{y}|) \rho(\mathbf{y}-\mathbf{x}) \mathrm{K}(\mathbf{y}-\mathbf{x}) \odot \frac{\phi(\mathbf{x})-\phi(\xi(\mathbf{x}))}{|\mathbf{y}-\mathbf{x}|} d \mathbf{y} .
\end{aligned}
$$

Using the estimate $|\mathbf{x}-\xi(\mathbf{x})| \leq \delta(\mathbf{x}) \leq|\mathbf{x}-\mathbf{y}|$ when $\delta(\mathbf{x}) \leq|\mathbf{x}-\mathbf{y}|$, we have that for any $\mathbf{x} \in \Omega$,

$$
\begin{aligned}
& \left|\int_{\Omega} \chi_{[\delta(\mathbf{x}), \infty)}(|\mathbf{x}-\mathbf{y}|) \rho(\mathbf{y}-\mathbf{x}) \mathrm{K}(\mathbf{y}-\mathbf{x}) \odot \frac{\phi(\mathbf{x})}{|\mathbf{y}-\mathbf{x}|} d \mathbf{y}\right| \\
& \quad \leq C\|\nabla \phi\|_{L^{\infty}(\Omega)} \int_{\Omega} \chi_{[\delta(\mathbf{x}), \infty)}(|\mathbf{x}-\mathbf{y}|) \frac{|\mathbf{x}-\xi(\mathbf{x})|}{|\mathbf{x}-\mathbf{y}|} \rho(\mathbf{x}-\mathbf{y}) d \mathbf{y} \leq C\|\nabla \boldsymbol{\phi}\|_{L^{\infty}(\Omega)}\|\rho\|_{L^{1}}
\end{aligned}
$$

as desired.

Remark 2.4. Combining the estimate in Lemma 2.3 with the identity (2.3), we obtain that there exists a positive constant $C$ such that for any $\phi \in C_{c}^{1}\left(\Omega ; \mathbb{R}^{d}\right)$ and any $\mathrm{x} \in \Omega$

$$
\sup _{\epsilon>0}\left|\int_{\Omega \backslash B_{\epsilon}(\mathbf{x})} \rho(\mathbf{y}-\mathbf{x}) \mathrm{K}(\mathbf{y}-\mathbf{x}) \odot \frac{\phi(\mathbf{x})+\boldsymbol{\phi}(\mathbf{y})}{|\mathbf{x}-\mathbf{y}|} d \mathbf{y}\right| \leq C\|\rho\|_{L^{1}}\|\nabla \boldsymbol{\phi}\|_{L^{\infty}} .
$$

This estimate will be used later.

A natural follow up of Lemma 2.3 is the continuity of operators $\mathbb{D}_{\rho}^{+}$and $\mathbb{D}_{\rho}^{-}$in Sobolev spaces given below.

Lemma 2.5.

(1) The map $\mathbb{D}_{\rho}^{-}(\cdot)$ is a bounded operator from $W^{1, p}\left(\Omega ; \mathbb{R}^{d}\right)$ to $L^{p}\left(\Omega ; \mathbb{R}^{d}\right)$ with the estimate that

$$
\left\|\mathbb{D}_{\rho}^{-}(\phi)\right\|_{L^{p}} \leq C\|\rho\|_{L^{1}}\|\nabla \phi\|_{L^{p}} .
$$

(2) The map $\mathbb{D}_{\rho}^{+}(\cdot)$ is a bounded operator from $W_{0}^{1, p}\left(\Omega ; \mathbb{R}^{d}\right)$ to $L^{p}\left(\Omega ; \mathbb{R}^{d \times d}\right)$ with the estimate that

$$
\left\|\mathbb{D}_{\rho}^{+}(\phi)\right\|_{L^{p}} \leq C\|\rho\|_{L^{1}}\|\nabla \phi\|_{L^{p}} .
$$

Proof. Again the estimate corresponding to $\mathbb{D}_{\rho}^{-}$can be obtained in the same way as the estimates in Proposition 2.1. Moreover, the estimate for $\mathbb{D}_{\rho}^{+}$will use the relation $(2.1)$ between $\mathbb{D}_{\rho}^{-}$and $\mathbb{D}_{\rho}^{+}$. As a consequence, it suffices to show that

$$
\int_{\Omega} \chi_{[\delta(\mathbf{x}), \infty)}(|\mathbf{x}-\mathbf{y}|) \rho(\mathbf{y}-\mathbf{x}) \mathrm{K}(\mathbf{y}-\mathbf{x}) \odot \frac{\phi(\mathbf{x})}{|\mathbf{y}-\mathbf{x}|} d \mathbf{y}
$$

belongs to $L^{p}\left(\Omega ; \mathbb{R}^{d \times d}\right)$ for $\phi \in W_{0}^{1, p}\left(\Omega ; \mathbb{R}^{d}\right)$ with the correct estimate. To that end,

$$
\begin{aligned}
& \left(\int_{\Omega}\left|\int_{\Omega} \chi_{[\delta(\mathbf{x}), \infty)}(|\mathbf{x}-\mathbf{y}|) \rho(\mathbf{y}-\mathbf{x}) \mathrm{K}(\mathbf{y}-\mathbf{x}) \odot \frac{\boldsymbol{\phi}(\mathbf{x})}{|\mathbf{y}-\mathbf{x}|} d \mathbf{y}\right|^{p} d \mathbf{x}\right)^{1 / p} \\
& \quad \leq \int_{\mathbb{R}^{d}} \rho(\boldsymbol{\xi})\left(\int_{\Omega} \chi_{[\delta(\mathbf{x}), \infty)}(|\boldsymbol{\xi}|) \frac{|\boldsymbol{\phi}(\mathbf{x})|^{p}}{|\boldsymbol{\xi}|^{p}} d \mathbf{x}\right)^{1 / p} d \boldsymbol{\xi} \\
& \quad \leq C\|\rho\|_{L^{1}}\left(\int_{\Omega} \frac{|\boldsymbol{\phi}(\mathbf{x})|^{p}}{\delta(\mathbf{x})^{p}} d \mathbf{x}\right)^{1 / p} \leq C\|\rho\|_{L^{1}}\|\nabla \boldsymbol{\phi}\|_{L^{p}}
\end{aligned}
$$

where Hardy's inequality is used in the last inequality. 
Nonlocal integration by parts. For functions in classical Sobolev spaces, functions of bounded variation and functions of bounded deformation, we have seen that $\mathbb{G}_{\rho}^{\mathrm{k}} \mathbf{u}$ is well defined and agrees with the Lebesgue integral of a directed weighted difference quotient. We may also interpret $\mathbb{G}_{\rho}^{k} \mathbf{u}$ in the broader sense as a distribution. Below we define this notion.

Definition 2.6. Given $\mathbf{u} \in L^{1}\left(\Omega ; \mathbb{R}^{d}\right)$, we define the distributional nonlocal gradient second order tensor $\mathfrak{G}_{\rho}^{\mathrm{k}} \mathbf{u}$ as

$$
\left\langle\mathfrak{G}_{\rho}^{\mathrm{k}} \mathbf{u}, \boldsymbol{\phi}\right\rangle:=-\int_{\Omega} \mathbb{D}_{\rho}^{+}(\phi)(\mathbf{x}) \mathbf{u}(\mathbf{x}) d \mathbf{x}, \quad \forall \boldsymbol{\phi} \in C_{c}^{\infty}\left(\Omega ; \mathbb{R}^{d}\right) .
$$

Note that $\mathfrak{G}_{\rho}^{\mathrm{k}} \mathbf{u}$ is indeed a distribution as can be seen from the estimate that

$$
\left|\left\langle\mathfrak{G}_{\rho}^{\mathrm{k}} \mathbf{u}, \phi\right\rangle\right| \leq C\|\nabla \phi\|_{L^{\infty}}\|\mathbf{u}\|_{L^{1}} .
$$

where we have used Lemma 2.3. Moreover the next theorem shows that for a class of vector fields $\mathbf{u}$, the distributional nonlocal gradient is precisely the same as the one given by the pointwise definition.

Theorem 2.7 (Nonlocal integration by parts). Let $\mathbf{u} \in L^{1}\left(\Omega ; \mathbb{R}^{d}\right)$ and $\rho(\mathbf{x}-\mathbf{y})\left|\mathrm{K}(\mathbf{x}-\mathbf{y}) \mathcal{D}_{*} \mathbf{u}(\mathbf{y}, \mathbf{x})\right|$ be an integrable function in $\Omega \times \Omega$. Then $\mathbb{G}_{\rho}^{\mathrm{k}} \mathbf{u} \in L^{1}\left(\Omega ; \mathbb{R}^{d \times d}\right)$ and for any $\phi \in C_{c}^{1}\left(\Omega ; \mathbb{R}^{d}\right)$

$$
\int_{\Omega} \mathbb{G}_{\rho}^{\mathrm{k}} \mathbf{u}(\mathbf{x}) \boldsymbol{\phi}(\mathbf{x}) d \mathbf{x}=-\int_{\Omega} \mathbb{D}_{\rho}^{+}(\phi)(\mathbf{x}) \mathbf{u}(\mathbf{x}) d \mathbf{x} .
$$

In this case, by definition, the distributional nonlocal gradient $\mathfrak{G}_{\rho}^{k} \mathbf{u}$ is precisely the tensor $\mathbb{G}_{\rho}^{\mathrm{k}} \mathbf{u}$.

Proof. Noting the integrability assumption, we have by Lebesgue dominated theorem that

$$
\int_{\Omega} \mathbb{G}_{\rho}^{\mathrm{k}} \mathbf{u}(\mathbf{x}) \boldsymbol{\phi}(\mathbf{x}) d \mathbf{x}=\lim _{\epsilon \rightarrow 0} \iint_{\Omega_{\epsilon}^{2}} \rho(\mathbf{y}-\mathbf{x})\left[\mathrm{K}(\mathbf{y}-\mathbf{x}) \frac{\mathbf{u}(\mathbf{y})-\mathbf{u}(\mathbf{x})}{|\mathbf{y}-\mathbf{x}|}\right] \boldsymbol{\phi}(\mathbf{x}) d \mathbf{y} d \mathbf{x}
$$

where

$$
\Omega_{\epsilon}^{2}=\Omega \times\left(\Omega \backslash B_{\epsilon}(\mathbf{x})\right)=(\Omega \times \Omega) \backslash\left\{(\mathbf{x}, \mathbf{y}) \in \mathbb{R}^{2 d}|| \mathbf{x}-\mathbf{y} \mid \leq \epsilon\right\} .
$$

From (2.4), we may also apply Lebesgue dominated theorem to obtain that

$$
\int_{\Omega} \mathbb{D}_{\rho}^{+}(\boldsymbol{\phi})(\mathbf{x}) \mathbf{u}(\mathbf{x}) d \mathbf{x}=-\lim _{\epsilon \rightarrow 0} \iint_{\Omega_{\epsilon}^{2}} \rho(\mathbf{y}-\mathbf{x})[\mathrm{K}(\mathbf{y}-\mathbf{x}) \mathbf{u}(\mathbf{x})] \frac{\phi(\mathbf{y})+\phi(\mathbf{x})}{|\mathbf{y}-\mathbf{x}|} d \mathbf{y} d \mathbf{x}
$$

where we used the identity $[\mathrm{K} \odot \boldsymbol{\phi}] \mathbf{u}=[\mathrm{Ku}] \boldsymbol{\phi}$.

Therefore, to prove the theorem, it suffices to show that for all $\epsilon>0$,

$$
\begin{aligned}
& \iint_{\Omega_{\epsilon}^{2}} \rho(\mathbf{y}-\mathbf{x})\left[\mathrm{K}(\mathbf{y}-\mathbf{x}) \frac{\mathbf{u}(\mathbf{y})-\mathbf{u}(\mathbf{x})}{|\mathbf{y}-\mathbf{x}|}\right] \boldsymbol{\phi}(\mathbf{x}) d \mathbf{y} d \mathbf{x} \\
& \quad=-\iint_{\Omega_{\epsilon}^{2}} \rho(\mathbf{y}-\mathbf{x})[\mathrm{K}(\mathbf{y}-\mathbf{x}) \mathbf{u}(\mathbf{x})] \frac{\phi(\mathbf{y})+\boldsymbol{\phi}(\mathbf{x})}{|\mathbf{y}-\mathbf{x}|} d \mathbf{y} d \mathbf{x} .
\end{aligned}
$$

To that end, we use the symmetry of the domain of integration $\Omega_{\epsilon}^{2}$, integrability of the function $\mathbf{u}$ and Fubini's theorem whose application is justified as the integrand is integrable once we truncate the singularity. Indeed,

$$
\begin{aligned}
& \iint_{\Omega_{\epsilon}^{2}} \rho(\mathbf{y}-\mathbf{x})\left[\mathrm{K}(\mathbf{y}-\mathbf{x}) \frac{\mathbf{u}(\mathbf{y})-\mathbf{u}(\mathbf{x})}{|\mathbf{y}-\mathbf{x}|}\right] \boldsymbol{\phi}(\mathbf{x}) d \mathbf{y} d \mathbf{x} \\
& =\iint_{\Omega_{\epsilon}^{2}} \rho(\mathbf{y}-\mathbf{x})\left[\mathrm{K}(\mathbf{y}-\mathbf{x}) \frac{\mathbf{u}(\mathbf{y})}{|\mathbf{y}-\mathbf{x}|}\right] \boldsymbol{\phi}(\mathbf{x}) d \mathbf{y} d \mathbf{x}-\iint_{\Omega_{\epsilon}^{2}} \rho(\mathbf{y}-\mathbf{x})\left[\mathrm{K}(\mathbf{y}-\mathbf{x}) \frac{\mathbf{u}(\mathbf{x})}{|\mathbf{y}-\mathbf{x}|}\right] \boldsymbol{\phi}(\mathbf{x}) d \mathbf{y} d \mathbf{x} \\
& =-\iint_{\Omega_{\epsilon}^{2}} \rho(\mathbf{y}-\mathbf{x})[\mathrm{K}(\mathbf{y}-\mathbf{x}) \mathbf{u}(\mathbf{x})] \frac{\phi(\mathbf{y})}{|\mathbf{y}-\mathbf{x}|} d \mathbf{y} d \mathbf{x}-\iint_{\Omega_{\epsilon}^{2}} \rho(\mathbf{y}-\mathbf{x})[\mathrm{K}(\mathbf{y}-\mathbf{x}) \mathbf{u}(\mathbf{x})] \frac{\phi(\mathbf{x})}{|\mathbf{y}-\mathbf{x}|} d \mathbf{y} d \mathbf{x}
\end{aligned}
$$

where in the last equality we interchanged the role of $\mathbf{x}$ and $\mathbf{y}$ and interchanged the integrals in the first term of the right hand side. By combining the last two terms, the theorem is proved. 
Remark 2.8. Given the smooth matrix function $\mathbf{\Phi}=\left(\Phi_{i j}\right) \in C_{c}^{1}\left(\Omega ; \mathbb{R}^{d \times d}\right)$ the nonlocal distributional gradient tensor $\mathfrak{G}_{\rho}^{\mathrm{k}}(\mathbf{u})$ satisfies the identity

$$
\begin{aligned}
\left\langle\mathfrak{G}_{\rho}^{\mathrm{k}}(\mathbf{u}): \mathbf{\Phi}\right\rangle & :=\sum_{i, j}^{d}\left\langle\mathfrak{G}_{\rho}^{\mathrm{k}}(\mathbf{u})_{i j}, \Phi_{i j}\right\rangle=\sum_{i}^{d}\left\langle\mathfrak{G}_{\rho}^{\mathrm{k}}(\mathbf{u})_{i},\left[\Phi^{t}\right]_{\cdot i}\right\rangle=-\sum_{i}^{d} \int_{\Omega} \mathbb{D}_{\rho}^{+}\left(\left[\Phi^{t}\right]_{,, i}\right)(\mathbf{x}) \mathbf{u}(\mathbf{x}) d \mathbf{x} \\
& =-\sum_{i, j}^{d} \int_{\Omega} \rho(\mathbf{y}-\mathbf{x})[\mathrm{K}(\mathbf{y}-\mathbf{x}) \mathbf{u}(\mathbf{x})]_{i j} \frac{\Phi_{i j}(\mathbf{x})+\Phi_{i j}(\mathbf{y})}{|\mathbf{y}-\mathbf{x}|} d \mathbf{x} d \mathbf{y}
\end{aligned}
$$

where the notation $\left\langle\mathfrak{G}_{\rho}^{\mathrm{k}}(\mathbf{u})_{i j}, \Phi_{i j}\right\rangle$ represents the action of the $i j^{\text {th }}$ entry of the distribution $\mathfrak{G}_{\rho}^{\mathrm{k}}(\mathbf{u})$ over the smooth function $\Phi_{i j}$.

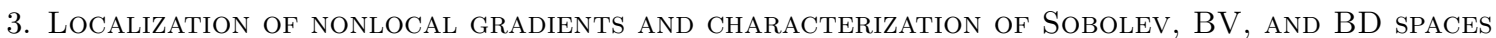

In this section we focus on the localization and characterization of nonlocal function spaces of interest based on the nonlocal vector calculus [18] and nonlocal operators.

Localization. We begin the discussion on localization by considering a sequence of radial kernel functions $\left\{\rho_{n}\right\}$ with the property that

$$
\rho_{n} \geq 0, \quad \int_{\mathbb{R}^{d}} \rho_{n}(\boldsymbol{\xi})=d, \quad \text { and for all } \delta>0, \lim _{n \rightarrow \infty} \int_{|\boldsymbol{\xi}|>\delta} \rho_{n}(\boldsymbol{\xi}) d \boldsymbol{\xi}=0 .
$$

The properties in (3.1) essentially say that $\left\{\rho_{n}\right\}$ is a sequence of functions that converge to the Dirac-Delta function. For a list of examples of sequence of $\left\{\rho_{n}\right\}$ satisfying $(3.1)$, see $[9,30]$. Corresponding to each sequence $\left\{\rho_{n}\right\}$, we will study the limiting operators associated with $\mathbb{G}_{\rho_{n}}^{\mathrm{k}}, \mathbb{D}_{\rho_{n}}^{+}$, and $\mathbb{D}_{\rho_{n}}^{-}$. For notational simplicity, we use $\mathbb{G}_{n}^{\mathrm{k}}, \mathbb{D}_{n}^{+}$, and $\mathbb{D}_{n}^{-}$to denote these operators corresponding to kernels $\left\{\rho_{n}\right\}$.

Let us first introduce a fourth order tensor $\mathrm{C}$ given by

$$
\mathrm{C}=f_{B_{1}(0)} \mathrm{K}(\mathbf{z}) \otimes \frac{\mathbf{z}}{|\mathbf{z}|} d \mathbf{z} .
$$

A simple calculation shows that for any matrix $\mathbb{A}$,

$$
\lim _{n \rightarrow \infty} \int_{B_{1}(0)} \rho_{n}(\mathbf{z}) \mathrm{K}(\mathbf{z}) \mathbb{A} \frac{\mathbf{z}}{|\mathbf{z}|} d \mathbf{z}=\lim _{n \rightarrow \infty} \int_{B_{1}(0)} \rho_{n}(\mathbf{z}) \mathrm{K}(\mathbf{z}) \frac{\mathbb{A} \mathbf{z}}{|\mathbf{z}|} d \mathbf{z}=\mathrm{CA}^{t} .
$$

Note that for any fourth order tensor $C=\left(C_{i j k l}\right)$ and a matrix $\mathbb{B}=\left(B_{i j}\right)$, the quantity $C \mathbb{B}$ is a matrix whose $i j^{t h}$ entry is given by

$$
[\mathrm{CB}]_{i j}=\sum_{k l} C_{i j k l} B_{l k} .
$$

Lemma 3.1. For any matrix $\mathbb{A}$, the sequence of matrix functions

$$
\mathbf{x} \mapsto \int_{\Omega} \rho_{n}(\mathbf{y}-\mathbf{x}) \mathrm{K}(\mathbf{y}-\mathbf{x}) \mathbb{A} \frac{\mathbf{y}-\mathbf{x}}{|\mathbf{y}-\mathbf{x}|} d \mathbf{y}
$$

converges to the matrix $\mathrm{CA}^{t}$ locally uniformly in $\Omega$.

Proof. We will show that for each $\mathbf{x} \in \Omega$ and any matrix $\mathbb{A}$,

$$
\lim _{n \rightarrow \infty} \int_{\Omega} \rho_{n}(\mathbf{y}-\mathbf{x}) \mathrm{K}(\mathbf{y}-\mathbf{x}) \mathbb{A} \frac{\mathbf{y}-\mathbf{x}}{|\mathbf{y}-\mathbf{x}|} d \mathbf{y}=\mathrm{CA}^{t},
$$

where the convergence is locally uniform in $\Omega$. Indeed, recalling that $\delta(\mathbf{x})$ denoted dist $(\mathbf{x}, \partial \Omega)$, we have that the ball centered at $\mathbf{x}$ with radius, $\delta(\mathbf{x}) B(\mathbf{x}, \delta(\mathbf{x})) \subset \Omega$ and

$$
\begin{aligned}
\int_{\Omega} \rho_{n}(\mathbf{y}-\mathbf{x}) \mathrm{K}(\mathbf{y}-\mathbf{x}) \mathbb{A} \frac{\mathbf{y}-\mathbf{x}}{|\mathbf{y}-\mathbf{x}|} d \mathbf{y}= & \int_{B(\mathbf{x}, \delta(\mathbf{x}))} \rho_{n}(\mathbf{y}-\mathbf{x}) \mathrm{K}(\mathbf{y}-\mathbf{x}) \mathbb{A} \frac{\mathbf{y}-\mathbf{x}}{|\mathbf{y}-\mathbf{x}|} d \mathbf{y} \\
& +\int_{\Omega \backslash B(\mathbf{x}, \delta(\mathbf{x}))} \rho_{n}(\mathbf{y}-\mathbf{x}) \mathrm{K}(\mathbf{y}-\mathbf{x}) \mathbb{A} \frac{\mathbf{y}-\mathbf{x}}{|\mathbf{y}-\mathbf{x}|} d \mathbf{y} .
\end{aligned}
$$


The second term on the right hand side can be estimated as

$$
\left|\int_{\Omega \backslash B(\mathbf{x}, \delta(\mathbf{x}))} \rho_{n}(\mathbf{y}-\mathbf{x}) \mathrm{K}(\mathbf{y}-\mathbf{x}) \mathbb{A} \frac{\mathbf{y}-\mathbf{x}}{|\mathbf{y}-\mathbf{x}|} d \mathbf{y}\right| \leq C|\mathbb{A}| \int_{\Omega \backslash B(\mathbf{x}, \delta(\mathbf{x}))} \rho_{n}(\mathbf{y}-\mathbf{x}) d \mathbf{y} \rightarrow 0 \quad \text { as } n \rightarrow \infty .
$$

The above convergence is uniform for $\mathbf{x}$ in a subset of $\Omega$ which is compactly contained in $\Omega$. Again using (3.1) and reasoning as above, we have

$$
\begin{aligned}
\lim _{n \rightarrow \infty} \int_{\Omega} \rho_{n}(\mathbf{y}-\mathbf{x}) \mathrm{K}(\mathbf{y}-\mathbf{x}) \mathbb{A} \frac{\mathbf{y}-\mathbf{x}}{|\mathbf{y}-\mathbf{x}|} d \mathbf{y} & =\lim _{n \rightarrow \infty} \int_{B(\mathbf{x}, \delta(\mathbf{x}))} \rho_{n}(\mathbf{y}-\mathbf{x}) \mathrm{K}(\mathbf{y}-\mathbf{x}) \mathbb{A} \frac{\mathbf{y}-\mathbf{x}}{|\mathbf{y}-\mathbf{x}|} d \mathbf{y} \\
& =\lim _{n \rightarrow \infty} \int_{B_{1}(\mathbf{x})} \rho_{n}(\mathbf{y}-\mathbf{x}) \mathrm{K}(\mathbf{y}-\mathbf{x}) \mathbb{A} \frac{\mathbf{y}-\mathbf{x}}{|\mathbf{y}-\mathbf{x}|} d \mathbf{y} \\
& =\lim _{n \rightarrow \infty} \int_{B_{1}(\mathbf{0})} \rho_{n}(\mathbf{z}) \mathrm{K}(\mathbf{z}) \mathbb{A} \frac{\mathbf{z}}{|\mathbf{z}|} d \mathbf{z}=\mathrm{C} \mathbb{A}^{t}
\end{aligned}
$$

where in the last equality we used the identity that $\mathrm{K}[\mathbb{A} \mathbf{v}]=[\mathrm{K} \otimes \mathbf{v}] \mathbb{A}^{t}$.

We would like to mention that the sequence of operators $\mathbb{G}_{n}^{\mathrm{k}}$ is uniformly bounded from $W^{1, p}\left(\Omega ; \mathbb{R}^{d}\right)$ to $L^{p}\left(\Omega ; \mathbb{R}^{d \times d}\right)$ in the operator norm. Indeed, from the part 2 of Proposition 2.1 , we see that

$$
\left\|\mathbb{G}_{n}^{\mathrm{k}} \mathbf{u}\right\|_{L^{p}} \leq C\left\|\rho_{n}\right\|_{L^{1}}\|\nabla \mathbf{u}\|_{L^{p}} \leq C\|\nabla \mathbf{u}\|_{L^{p}}
$$

where we used the assumption in (3.1). A similar argument can be made to conclude that the sequence of operators $\mathbb{D}_{n}^{+}$, and $\mathbb{D}_{n}^{-}$are uniformly bounded in their respective domain. The following theorem proves the pointwise limit of the sequence of operators $\mathbb{G}_{n}^{k}$ in two different topologies.

Theorem 3.2. Suppose that $\Omega \subset \mathbb{R}^{d}$ is open, bounded, and sufficiently smooth. Assume also that $1 \leq p<\infty$, $\rho_{n}$ satisfy (3.1) and $\mathrm{C}$ is given in (3.2). Then the following properties hold.

1. For any $\mathbf{u} \in C^{1}\left(\bar{\Omega} ; \mathbb{R}^{d}\right), \mathbb{G}_{n}^{\mathrm{k}} \mathbf{u} \rightarrow \mathrm{C}(\nabla \mathbf{u})^{t}$ locally uniformly as $n \rightarrow \infty$. If $\mathbf{u} \in C_{c}^{1}\left(\Omega ; \mathbb{R}^{d}\right)$, then the convergence is uniform.

2. For any $\mathbf{u} \in W^{1, p}\left(\Omega ; \mathbb{R}^{d}\right), \mathbb{G}_{n}^{\mathrm{k}} \mathbf{u} \rightarrow \mathrm{C}(\nabla \mathbf{u})^{t}$ in $L^{p}\left(\Omega ; \mathbb{R}^{d}\right)$ as $n \rightarrow \infty$.

Proof. Let $\mathbf{u} \in C^{1}\left(\bar{\Omega} ; \mathbb{R}^{d}\right)$ and $K \subset \Omega$ be compact. We will show that $\mathbb{G}_{n}^{\mathrm{k}} \mathbf{u}(\mathbf{x}) \rightarrow C \nabla \mathbf{u}(\mathbf{x})^{t}$ uniformly for $\mathbf{x} \in K$ as $n \rightarrow \infty$. Let us show first that as $n \rightarrow \infty$, the sequence of functions

$$
J_{n}(\mathbf{x}):=\left|\int_{\Omega} \rho_{n}(\mathbf{y}-\mathbf{x}) \mathrm{K}(\mathbf{y}-\mathbf{x}) \frac{\mathbf{u}(\mathbf{y})-\mathbf{u}(\mathbf{x})-\nabla \mathbf{u}(\mathbf{x})(\mathbf{y}-\mathbf{x})}{|\mathbf{x}-\mathbf{y}|} d \mathbf{y}\right|
$$

tends to zero uniformly for $\mathbf{x} \in \Omega$. To that end, let $\epsilon>0$ be arbitrary. Since $\nabla \mathbf{u}$ is continuous on $\bar{\Omega}$, we can choose $\tau>0$ small so that for all $\mathbf{x}, \mathbf{y} \in \Omega$,

$$
|\nabla \mathbf{u}(\mathbf{x})-\nabla \mathbf{u}(\mathbf{y})|<\epsilon \text { whenever }|\mathbf{x}-\mathbf{y}|<\tau \text {. }
$$

Then using the fact that $\sup _{\mathbf{x}, \mathbf{y} \in \bar{\Omega}}\left|\mathcal{D}_{*}(\mathbf{u})(\mathbf{y}, \mathbf{x})\right| \leq\|\nabla \mathbf{u}\|_{L^{\infty}}$, we have the estimate that

$$
\begin{aligned}
J_{n}(\mathbf{x}) & \leq\left|\int_{B_{\tau}(\mathbf{x}) \cap \Omega} \rho_{n}(\mathbf{y}-\mathbf{x}) \mathrm{K}(\mathbf{y}-\mathbf{x}) \frac{\mathbf{u}(\mathbf{y})-\mathbf{u}(\mathbf{x})-\nabla \mathbf{u}(\mathbf{x})(\mathbf{y}-\mathbf{x})}{|\mathbf{x}-\mathbf{y}|} d \mathbf{y}\right| \\
& +2 C\|\nabla \mathbf{u}\|_{L^{\infty}} \int_{|\mathbf{x}-\mathbf{y}| \geq \tau} \rho_{n}(\mathbf{y}-\mathbf{x}) d \mathbf{y} .
\end{aligned}
$$

Using the mean value theorem, for every $\mathbf{y} \in B_{\tau}(\mathbf{x}) \cap \Omega$, there exists $\xi(\mathbf{x}, \mathbf{y}) \in B_{\tau}(\mathbf{x}) \cap \Omega$ such that

$$
\mathbf{u}(\mathbf{y})-\mathbf{u}(\mathbf{x})=\nabla \mathbf{u}(\xi(\mathbf{x}, \mathbf{y}))(\mathbf{y}-\mathbf{x})
$$

which implies the estimate

$$
J_{n}(\mathbf{x}) \leq C \epsilon \int_{|\mathbf{z}|<\tau} \rho_{n}(|\mathbf{z}|) d \mathbf{z}+2 C\|\nabla \mathbf{u}\|_{L^{\infty}(\Omega)} \int_{|\mathbf{z}| \geq \tau} \rho_{n}(|\mathbf{z}|) d \mathbf{z} .
$$

From the last estimate we obtain that $\lim _{n \rightarrow \infty} \sup _{x \in \Omega} J_{n}(\mathbf{x}) \leq C \epsilon$, and by sending $\epsilon \rightarrow 0$, we have the uniform convergence on $J_{n}(\mathbf{x})$ in $\Omega$. Finally, we obtain that for any $\mathbf{x} \in \Omega$

$$
\lim _{n \rightarrow \infty} \mathbb{G}_{n}^{\mathrm{k}} \mathbf{u}(\mathbf{x})=\lim _{n \rightarrow \infty} \int_{\Omega} \rho_{n}(\mathbf{y}-\mathbf{x}) \mathrm{K}(\mathbf{y}-\mathbf{x}) \frac{\nabla \mathbf{u}(\mathbf{x})(\mathbf{y}-\mathbf{x})}{|\mathbf{y}-\mathbf{x}|} d \mathbf{y}=\mathrm{C}(\nabla \mathbf{u}(\mathbf{x}))^{t}
$$


where by Lemma 3.1 , the convergence stated above is locally uniform in $\Omega$. if $\mathbf{u} \in C_{c}^{1}\left(\Omega ; \mathbb{R}^{d}\right)$, then the last convergence is uniform on the closure of $\operatorname{Supp}(\mathbf{u})$ and therefore uniform over $\Omega$.

To prove the second part of the theorem, let us show the estimate in the $L^{p}$-norm for $\mathbf{v} \in C^{1}\left(\bar{\Omega} ; \mathbb{R}^{d}\right)$. To that end, for each $\tau>0$, let us consider

$$
\left\|\mathbb{G}_{n}^{\mathrm{k}} \mathbf{v}-\mathrm{C} \nabla \mathbf{v}^{t}\right\|_{L^{p}}^{p}=\int_{\Omega_{\tau}}\left|\mathbb{G}_{n}^{\mathrm{k}} \mathbf{v}(\mathbf{x})-\mathrm{C} \nabla \mathbf{v}(\mathbf{x})^{t}\right|^{p} d \mathbf{x}+\int_{\Omega_{\backslash \Omega_{\tau}}}\left|\mathbb{G}_{n}^{\mathrm{k}} \mathbf{v}(\mathbf{x})-\mathrm{C} \nabla \mathbf{v}(\mathbf{x})^{t}\right|^{p} d \mathbf{x}
$$

where $\Omega_{\tau}=\{\mathbf{x} \in \Omega: \operatorname{dist}(\mathbf{x}, \partial \Omega)>\tau\}$. For fixed $\tau>0$, the first term goes to 0 as $n \rightarrow \infty$ by part a). The second term can be estimated as

$$
\left|\int_{\Omega \backslash \Omega_{\tau}}\right| \mathbb{G}_{n}^{\mathrm{k}} \mathbf{v}(\mathbf{x})-\left.C \nabla \mathbf{v}(\mathbf{x})^{t}\right|^{p} d \mathbf{x}\left|\leq C\|\nabla \mathbf{v}\|_{L^{\infty}}\right| \Omega \backslash \Omega_{\tau} \mid,
$$

which we can make as small as we wish by letting $\tau \rightarrow 0$. The conclusion of the theorem follows for any $\mathbf{v} \in$ $W^{1, p}\left(\Omega ; \mathbb{R}^{d}\right)$ using the fact that $C^{1}\left(\bar{\Omega} ; \mathbb{R}^{d}\right)$ is dense in $W^{1, p}\left(\Omega ; \mathbb{R}^{d}\right)$ and that $\mathbb{G}_{n}^{\mathrm{k}}$ is a sequence of uniformly bounded operators from $W^{1, p}\left(\Omega ; \mathbb{R}^{d}\right)$ to $L^{p}\left(\Omega ; \mathbb{R}^{d \times d}\right)$, see Proposition 2.1

Remark 3.3. In general, the local uniform convergence in part 1 of the above theorem cannot be globally uniform. Indeed, we may take $d=1, u(x)=x, x \in[-1,1]$. Then $u \in C^{\infty}([-1,1])$. However, taking $\rho_{n}(\xi)=\frac{n}{2} \chi_{[-1,1]}(n \xi)$ and $k_{1}(\xi)=\frac{\xi}{|\xi|}$, the function $G_{n}^{\mathrm{k}_{1}} u$ given by

$$
G_{n}^{\mathrm{k}_{1}} u(x)= \begin{cases}\frac{n x+1+n}{2}, & \text { if } x \in[-1,-1+1 / n] \\ 1, & \text { if } x \in[-1+1 / n, 1-1 / n] \\ \frac{n+1-n x}{2}, & \text { if } x \in[1-1 / n, 1],\end{cases}
$$

converges locally uniformly to 1 in $[-1,1]$, but not uniformly in $[-1,1]$.

The following lemma says that $\mathbb{D}_{n}^{+}$and $\mathbb{D}_{n}^{-}$are essentially the same in the limit.

Lemma 3.4. Let $\phi \in C_{c}^{1}\left(\Omega ; \mathbb{R}^{d}\right)$, and $\gamma=\operatorname{dist}(\operatorname{Supp}(\phi), \partial \Omega)>0$. Then for all $\mathbf{x} \in \Omega$,

$$
\left|\mathbb{D}_{n}^{+}(\phi)(\mathbf{x})-\mathbb{D}_{n}^{-}(\phi)(\mathbf{x})\right| \leq C\|\phi\|_{L^{\infty}} \frac{1}{\gamma} \int_{B_{\gamma}(\mathbf{0})^{C}} \rho_{n}(|\mathbf{z}|) d \mathbf{z} .
$$

Consequently, for any $\mathrm{x} \in \Omega$,

$$
\left|\mathbb{D}_{n}^{+}(\phi)(\mathbf{x})-\mathbb{D}_{n}^{-}(\phi)(\mathbf{x})\right| \rightarrow 0, \quad \text { as } n \rightarrow \infty .
$$

Moreover, if $\mathbf{u} \in L^{p}\left(\Omega ; \mathbb{R}^{d}\right)$ and $1 \leq p \leq \infty$, then

$$
\left\|\mathbb{D}_{n}^{+}(\phi) \mathbf{u}-\mathbb{D}_{n}^{-}(\phi) \mathbf{u}\right\|_{L^{p}} \rightarrow 0, \quad \text { as } n \rightarrow \infty .
$$

The proof is straightforward and uses the identity $(2.1)$ that relates $\mathbb{D}_{n}^{+}$and $\mathbb{D}_{n}^{-}$. The details are omitted. Furthermore, we can obtain the closed form of the limit of $\mathbb{D}_{n}^{+}(\phi)(\mathbf{x})$ for smooth $\phi$. Using a similar argument used before, for each $\mathrm{x} \in \Omega$

$$
\begin{aligned}
\lim _{n \rightarrow \infty} \mathbb{D}_{n}^{+}(\phi)(\mathbf{x})=\lim _{n \rightarrow \infty} \mathbb{D}_{n}^{-}(\phi)(\mathbf{x}) & =\lim _{n \rightarrow \infty} \int_{\Omega} \rho_{n}(\mathbf{y}-\mathbf{x}) \mathrm{K}(\mathbf{y}-\mathbf{x}) \odot \nabla \phi(\mathbf{x}) \frac{\mathbf{y}-\mathbf{x}}{|\mathbf{y}-\mathbf{x}|} d \mathbf{y} \\
& =\lim _{n \rightarrow \infty} \int_{B_{1}(\mathbf{x})} \rho_{n}(\mathbf{y}-\mathbf{x}) \mathrm{K}(\mathbf{y}-\mathbf{x}) \odot \nabla \boldsymbol{\phi}(\mathbf{x}) \frac{\mathbf{y}-\mathbf{x}}{|\mathbf{y}-\mathbf{x}|} d \mathbf{y} \\
& =\lim _{n \rightarrow \infty} \int_{B_{1}(\mathbf{0})} \rho_{n}(\mathbf{z}) \mathrm{K}(\mathbf{z}) \odot \nabla \boldsymbol{\phi}(\mathbf{x}) \frac{\mathbf{z}}{|\mathbf{z}|} d \mathbf{z} \\
& =f_{B_{1}(\mathbf{0})} \mathrm{K}(\mathbf{z}) \odot \nabla \boldsymbol{\phi}(\mathbf{x}) \frac{\mathbf{z}}{|\mathbf{z}|} d \mathbf{z} .
\end{aligned}
$$

Combining (3.4) with Lemma 3.4, we obtain the following corollary.

Corollary 3.5. Let $\phi \in C_{c}^{1}\left(\Omega ; \mathbb{R}^{d}\right)$ and $1 \leq p \leq \infty$. Then both sequences of matrix functions $\mathbb{D}_{n}^{+}(\phi)$ and $\mathbb{D}_{n}^{-}(\phi)$ converge to the map

in $L^{p}\left(\Omega ; \mathbb{R}^{d \times d}\right)$.

$$
\mathbf{x} \mapsto f_{B_{1}(\mathbf{0})} \mathrm{K}(\mathbf{z}) \odot \nabla \phi(\mathbf{x}) \frac{\mathbf{z}}{|\mathbf{z}|} d \mathbf{z}
$$


The last convergence in (3.4) motivates the introduction of the general class of function space that is based on the third order tensor K. For specialized $\mathrm{K}^{\prime} s$ we recover the classical Sobolev spaces, the spaces of functions of bounded variation and the space of functions of bounded deformation. The study of these functions is the main topic in the remainder of this section.

Distributional derivatives and function spaces associated with third order tensors. First, we recall the constant fourth order tensor $C$ defined in (3.2). For any smooth vector field $\mathbf{u}$, the matrix $C \nabla \mathbf{u}^{t}$ has entries which are linear combinations of the partial derivatives of $\mathbf{u}$. By definition and application of integration by parts, for any $\psi \in C_{c}^{1}(\Omega)$ and smooth $\mathbf{u}$, the $i j^{\text {th }}$ entry $\left[C \nabla \mathbf{u}^{t}\right]_{i j}$ satisfies the identity

$$
\begin{aligned}
\int_{\Omega}\left[C \nabla \mathbf{u}(\mathbf{x})^{t}\right]_{i j} \psi(\mathbf{x}) d \mathbf{x} & =f_{B_{1}(\mathbf{0})} \sum_{l, m=1}^{d} \int_{\Omega} \mathrm{K}_{i j l}(\mathbf{z}) \frac{z_{m}}{|\mathbf{z}|} \frac{\partial u_{l}(\mathbf{x})}{\partial_{x_{m}}} \psi(\mathbf{x}) d \mathbf{x} d \mathbf{z} \\
& =-\int_{\Omega} \sum_{l, m=1}^{d} f_{B_{1}(\mathbf{0})} \mathrm{K}_{i j l}(\mathbf{z}) u_{l}(\mathbf{x}) \frac{z_{m}}{|\mathbf{z}|} \frac{\partial \psi(\mathbf{x})}{\partial_{x_{m}}} d \mathbf{z} d \mathbf{x} .
\end{aligned}
$$

It is now natural to use the above identity to define a matrix of distributions associated with any integrable vector field $\mathbf{u}$ that agrees with $\left[\mathrm{C} \nabla \mathbf{u}(\mathbf{x})^{t}\right]_{i j}$ when $\mathbf{u}$ is smooth.

Definition 3.6 (K-distributional derivative). For a given integrable vector field $\mathbf{u}$, associated with any third order tensor field $\mathrm{K}$ satisfying $(1.2)$, we define the matrix distribution $D^{\mathrm{K}}(\mathbf{u})$, whose $i j^{\text {th }}$ entry is given by the action

$$
\left\langle\left[D^{\mathrm{K}}(\mathbf{u})\right]_{i j}, \psi\right\rangle:=-\int_{\Omega} f_{B_{1}(\mathbf{0})}[\mathrm{K}(\mathbf{z}) \mathbf{u}(\mathbf{x})]_{i j} \frac{\mathbf{z}}{|\mathbf{z}|} \cdot \nabla \psi(\mathbf{x}) d \mathbf{z} .
$$

for any $\psi \in C_{c}^{1}(\Omega)$.

It is not difficult to see that $D^{\mathrm{K}}(\mathbf{u})$ is a distribution with the estimate that, for a universal constant $C$,

$$
\left|\left\langle\left[D^{\mathrm{K}}(\mathbf{u})\right]_{i j}, \psi\right\rangle\right| \leq C\|\nabla \psi\|_{L^{\infty}}\|\mathbf{u}\|_{L^{1}}
$$

for all $\psi \in C_{c}^{1}(\Omega)$, and $\mathbf{u} \in L^{1}\left(\Omega ; \mathbb{R}^{d}\right)$. By definition, $\left[D^{\mathrm{K}}(\mathbf{u})\right]_{i j}=\left[\mathrm{C} \nabla \mathbf{u}^{t}\right]_{i j}$ in the sense of distributions and is a linear combination of the distributional partial derivatives of the components of $\mathbf{u}$. Let us introduce some more notation to rewrite the definition of the matrix distribution $D^{\mathrm{K}}(\mathbf{u})$. For any $\mathbf{u} \in L^{1}\left(\Omega ; \mathbb{R}^{d}\right)$, and each $i, j=1,2, \cdots, d$, we consider the linear operator $L^{\mathrm{k}}$ defined as

$$
\left[L^{\mathrm{k}}(\mathbf{u})\right]_{i j}(\mathbf{x})=f_{B_{1}(\mathbf{0})}[\mathrm{K}(\mathbf{z}) \mathbf{u}(\mathbf{x})]_{i j} \frac{\mathbf{z}}{|\mathbf{z}|} d \mathbf{z},
$$

which is actually linear combinations of components of the vector field $\mathbf{u}$. Then it is clear that for any $\psi \in C_{c}^{1}(\Omega)$,

$$
\left\langle\left[D^{\mathrm{K}}(\mathbf{u})\right]_{i j}, \psi\right\rangle=-\int_{\Omega}\left[L^{\mathrm{k}}(\mathbf{u})\right]_{i j}(\mathbf{x}) \cdot \nabla \psi(\mathbf{x}) d \mathbf{x},
$$

that is, for each $i, j=1, \cdots, d,\left[D^{\mathrm{K}}(\mathbf{u})\right]_{i j}=\operatorname{div}\left(\left[L^{\mathrm{k}}(\mathbf{u})\right]_{i j}\right)$ in the sense of distributions. The new formulation makes it easy to see the action of the distribution $D^{\mathrm{K}}(\mathbf{u})$ on smooth vectors and matrix functions. Indeed, for any $\phi=\left(\phi_{1}, \cdots, \phi_{d}\right)^{t} \in C_{c}^{1}\left(\Omega ; \mathbb{R}^{d}\right)$, the action of $D^{\mathrm{K}}(\mathbf{u})$ on $\phi$ is a vector with the $i^{\text {th }}$ component defined as

$$
\left\langle D^{\mathrm{K}}(\mathbf{u}), \phi\right\rangle_{i}:=\sum_{j=1}^{d}\left\langle\left[D^{k}(\mathbf{u})\right]_{i j}, \phi_{j}\right\rangle=-\sum_{j=1}^{d} \int_{\Omega} L_{i, j}^{\mathrm{k}}(\mathbf{u})(\mathbf{x}) \cdot \nabla \phi_{j} d \mathbf{x} .
$$

Similarly, for any $\boldsymbol{\Phi}=\left(\Phi_{i j}\right) \in C_{c}^{1}\left(\Omega, \mathbb{R}^{d \times d}\right)$, the action of $D^{\mathrm{K}}(\mathbf{u})$, on $\boldsymbol{\Phi}$ is a scalar given by

$$
\left\langle D^{\mathrm{K}}(\mathbf{u}): \mathbf{\Phi}\right\rangle:=\sum_{i, j}^{d}\left\langle\left[D^{\mathrm{K}}(\mathbf{u})\right]_{i j}, \Phi_{i j}\right\rangle=-\sum_{i, j}^{d} \int_{\Omega} L_{i, j}^{\mathrm{k}}(\mathbf{u})(\mathbf{x}) \cdot \nabla \Phi_{i j}(\mathbf{x}) d \mathbf{x} .
$$

Remark 3.7. We can also use the above notation to extend the notion of $\mathrm{K}$ distributional derivatives to finite Radon measures. Given a vector valued finite Radon measure $\boldsymbol{\mu}$ on $\Omega$, we first note that for any $i, j=1,2, \cdots, d$,

$$
\left[L^{\mathrm{k}}(\boldsymbol{\mu})\right]_{i j}:=f_{B_{1}(\mathbf{0})}[\mathrm{K}(\mathbf{z}) \boldsymbol{\mu}]_{i j} \frac{\mathbf{z}}{|\mathbf{z}|} d \mathbf{z}
$$


is also a vector valued finite Radon measure $\boldsymbol{\mu}$ on $\Omega$. Consequently the quantity $\left[D^{\mathrm{K}}(\boldsymbol{\mu})\right]_{i j}$ defined as

$$
\left.\left\langle\left[D^{\mathrm{K}}(\boldsymbol{\mu})\right]_{i j}, \psi\right\rangle:=\int_{\Omega} \nabla \psi \cdot d\left[L^{\mathrm{k}}(\boldsymbol{\mu})\right]_{i j}\right]
$$

is a distribution.

We now use the above notion of K-distributional derivatives to define the following function spaces. For $1 \leq p \leq \infty$, the function space $\mathcal{W}_{\mathrm{K}}^{1, p}(\Omega)$ is defined as

$$
\mathcal{W}_{\mathrm{K}}^{1, p}(\Omega)=\left\{\mathbf{u} \in L^{p}\left(\Omega ; \mathbb{R}^{d}\right): \quad D^{\mathrm{K}}(\mathbf{u}) \in L^{p}\left(\Omega ; \mathbb{R}^{d \times d}\right)\right\} .
$$

It is not difficult to show that $\mathcal{W}_{\mathrm{k}}^{1, p}(\Omega)$ is a Banach space with the norm

$$
\|\mathbf{u}\|_{W_{k}}^{p}=\|\mathbf{u}\|_{L^{p}}^{p}+\left\|D^{\mathrm{K}}(\mathbf{u})\right\|_{L^{p}}^{p}=\|\mathbf{u}\|_{L^{p}}^{p}+\sum_{i, j}^{d}\left\|\left[D^{\mathrm{K}}(\mathbf{u})\right]_{i j}\right\|_{L^{p}}^{p} .
$$

Let us also introduce the function spaces of vector fields whose K-distributional derivative is a bounded measure, i.e.

$$
\mathcal{M}_{\mathrm{k}}(\Omega)=\left\{\mathbf{u} \in L^{1}\left(\Omega ; \mathbb{R}^{d}\right): \quad D^{\mathrm{K}}(\mathbf{u}) \in M\left(\Omega ; \mathbb{R}^{d \times d}\right)\right\}
$$

where $M\left(\Omega ; \mathbb{R}^{d \times d}\right)$ is the space of matrix Radon measures with finite total variation. Again $\mathcal{M}_{\mathrm{k}}(\Omega)$ is a Banach space with the norm

$$
\|\mathbf{u}\|_{M_{k}}=\|\mathbf{u}\|_{L^{1}}+\left|D^{\mathrm{K}}(\mathbf{u})\right|(\Omega)
$$

where $\left|D^{\mathrm{K}}(\mathbf{u})\right|(\Omega)$ is the total variation of the matrix valued Radon measure $D^{\mathrm{K}}(\mathbf{u})$ given by

$$
\left|D^{\mathrm{K}}(\mathbf{u})\right|(\Omega)=\sup \left\{\sum_{i, j}^{d} \int_{\Omega} \boldsymbol{\Phi}_{i j}(\mathbf{x}) d D_{i j}^{\mathrm{K}}(\mathbf{u}) \quad \mid \quad \mathbf{\Phi} \in C_{c}\left(\Omega ; \mathbb{R}^{d \times d}\right),\|\boldsymbol{\Phi}\|_{L^{\infty}} \leq 1\right\} .
$$

In many ways, the spaces $\mathcal{W}_{\mathrm{k}}^{1, p}(\Omega)$ and $\mathcal{M}_{\mathrm{k}}(\Omega)$ essentially behave like Sobolev and BV space respectively. We will list some properties of these function spaces that can easily be proved using techniques from the theory of Sobolev and BV spaces. The following result states smooth functions are dense in $\mathcal{W}_{\mathrm{k}}^{1, p}(\Omega)$.

Lemma 3.8. Suppose that $\Omega$ is bounded with smooth boundary. Let $1 \leq p<\infty$, Then given $\mathbf{u} \in \mathcal{W}_{\mathrm{k}}^{1, p}(\Omega)$, there exists a sequence $\mathbf{u}_{n} \in C^{\infty}\left(\Omega ; \mathbb{R}^{d}\right) \cap \mathcal{W}_{\mathrm{k}}^{1, p}(\Omega)$ such that

$$
\left\|\mathbf{u}_{n}-\mathbf{u}\right\|_{W_{k}} \rightarrow 0, \quad \text { as } n \rightarrow \infty .
$$

The proof follows standard arguments using partitions of unity, see the proof of Theorem 2 in section 5.3 .2 of [20] and is based on two main ingredients: standard approximation by mollifiers, and some kind of 'product rule'. For $1 \leq p<\infty$, given that each component of $D^{\mathrm{K}} \mathbf{u}$ is some linear combination of the distributional partial derivatives, standard approximation by mollifiers remains valid. For example, if $\eta_{\epsilon}(\mathbf{x})=\frac{1}{\epsilon^{d}} \eta(\mathbf{x} / \epsilon) \in C_{c}^{\infty}(\Omega)$ is a standard sequence of mollifiers, then for any $\mathbf{u} \in \mathcal{W}_{\mathrm{K}}^{1, p}(\Omega)$, we have

$$
\mathbf{u}^{\epsilon}=\eta_{\epsilon} * \mathbf{u}(\mathbf{x})=\left(\eta_{\epsilon} * u_{1}, \eta_{\epsilon} * u_{2}, \cdots \eta_{\epsilon} * u_{d}\right), \quad \text { defined on } \Omega_{\epsilon}=\{\mathbf{x} \in \Omega: \operatorname{dist}(\mathbf{x}, \partial \Omega)>\epsilon\}
$$

is in $C^{\infty}\left(\Omega_{\epsilon} ; \mathbb{R}^{d}\right)$ and satisfies the identity

$$
\left[D^{\mathrm{K}}\left(\mathbf{u}^{\epsilon}\right)\right]_{i j}=\eta_{\epsilon} *\left[D^{\mathrm{K}}(\mathbf{u})\right]_{i j}, \quad \text { for all } \mathbf{x} \in \Omega_{\epsilon} .
$$

Indeed, in this case, $\left[D^{\mathrm{K}}\left(\mathbf{u}^{\epsilon}\right)\right]_{i j}=\left[C \nabla \mathbf{u}^{\epsilon t}\right]_{i j}$ and we have that for $\mathbf{x} \in \Omega_{\epsilon}$

$$
\begin{aligned}
{\left[C \nabla \mathbf{u}^{\epsilon t}\right]_{i j}=\sum_{l, m}^{d} f_{B_{1}(\mathbf{0})} \mathrm{K}_{i j l}(\mathbf{z}) \frac{z_{m}}{|\mathbf{z}|} \frac{\partial u_{l}^{\epsilon}}{\partial x_{m}} d \mathbf{z} } & =\sum_{l, m}^{d} f_{B_{1}(\mathbf{0})} \mathrm{K}_{i j l}(\mathbf{z}) \frac{z_{m}}{|\mathbf{z}|} \frac{\partial}{\partial x_{m}}\left(\int_{\Omega} \eta_{\epsilon}(\mathbf{x}-\mathbf{y}) u_{l}^{\epsilon}(\mathbf{y}) d \mathbf{y}\right) d \mathbf{z} \\
& =-\sum_{l, m}^{d} f_{B_{1}(\mathbf{0})} \mathrm{K}_{i j l}(\mathbf{z}) \frac{z_{m}}{|\mathbf{z}|}\left(\int_{\Omega} \frac{\partial}{\partial y_{m}} \eta_{\epsilon}(\mathbf{x}-\mathbf{y}) u_{l}^{\epsilon}(\mathbf{y}) d \mathbf{y}\right) d \mathbf{z} .
\end{aligned}
$$

The last term can be rewritten and simplified as

$$
-\int_{\Omega} \nabla_{\mathbf{y}} \eta^{\epsilon}(\mathbf{x}-\mathbf{y}) \cdot\left[L^{\mathrm{k}}(\mathbf{u})\right]_{i j}(\mathbf{y}) d \mathbf{y}=\int_{\Omega} \eta^{\epsilon}(\mathbf{x}-\mathbf{y})\left[D^{\mathrm{K}}\left(\mathbf{u}^{\epsilon}\right)\right]_{i j}(\mathbf{y}) d \mathbf{y}=\eta^{\epsilon} *\left[D^{\mathrm{K}}\left(\mathbf{u}^{\epsilon}\right)\right]_{i j}(\mathbf{x})
$$


where we used the fact that for $\mathbf{x} \in \Omega_{\epsilon}$, the function $\mathbf{y} \mapsto \eta^{\epsilon}(\mathbf{x}-\mathbf{y})$ is in $C_{c}^{1}(\Omega)$. We may now follow classical arguments to show that $\mathbf{u}^{\epsilon} \rightarrow \mathbf{u}$ locally in the $\mathcal{W}_{\mathrm{k}}$-norm defined above. It is also true that if $\zeta \in C_{c}^{1}(\Omega)$ and $\mathbf{u} \in \mathcal{W}_{\mathrm{k}}^{1, p}(\Omega)$, then we have the product $\zeta \mathbf{u} \in \mathcal{W}_{\mathrm{k}}^{1, p}(\Omega)$ with the product rule

$$
\left[D^{\mathrm{K}}(\zeta \mathbf{u})\right]_{i j}(\mathbf{x})=\zeta(\mathbf{x})\left[D^{\mathrm{K}}(\mathbf{u})\right]_{i j}(\mathbf{x})+\left[L^{\mathrm{k}}(\mathbf{u})\right]_{i j}(\mathbf{x}) \cdot \nabla \zeta(\mathbf{x}) .
$$

For functions in $\mathcal{M}_{\mathrm{k}}(\Omega)$, just like $B V$ functions (see [3]), the total variation can be computed via the variation introduced below.

Lemma 3.9. For $\mathbf{u} \in L^{1}\left(\Omega ; \mathbb{R}^{d}\right), \mathbf{u} \in \mathcal{M}_{\mathrm{k}}(\Omega)$ if and only if $\operatorname{Var}_{k}(\mathbf{u}, \Omega)<\infty$ where

$$
\operatorname{Var}_{k}(\mathbf{u}, \Omega)=\sup \left\{\sum_{i, j} \int_{\Omega} L_{i, j}^{\mathrm{k}}(\mathbf{u})(\mathbf{x}) \cdot \nabla \boldsymbol{\Phi}_{i j}(\mathbf{x}) d \mathbf{x}: \mathbf{\Phi} \in C_{c}^{1}\left(\Omega ; \mathbb{R}^{d \times d}\right),\|\boldsymbol{\Phi}\|_{L^{\infty}} \leq 1\right\} .
$$

Moreover, the total variation of the matrix valued measure $\left|D^{\mathrm{k}}(\mathbf{u})\right|(\Omega)$ coincides with the variation $\operatorname{Var}(\mathbf{u}, \Omega)<\infty$.

Proof. Suppose that $\mathbf{u} \in \mathcal{M}_{\mathrm{k}}(\Omega)$. Then for each $\boldsymbol{\Phi} \in C_{c}^{1}\left(\Omega ; \mathbb{R}^{d \times d}\right)$, by (3.5) and by definition we have that

$$
\sum_{i, j} \int_{\Omega} L_{i, j}^{\mathrm{k}}(\mathbf{u})(\mathbf{x}) \cdot \nabla \boldsymbol{\Phi}_{i j}(\mathbf{x}) d \mathbf{x}=-\sum_{i, j}^{d} \int_{\Omega} \boldsymbol{\Phi}_{i j}(\mathbf{x}) d D_{i j}^{\mathrm{K}}(\mathbf{u}) .
$$

Thus, after taking the supremum over all smooth $\mathbf{\Phi}$ with $\|\mathbf{\Phi}\|_{L^{\infty}} \leq 1$, we obtain that

$$
\operatorname{Var}(\mathbf{u}, \Omega) \leq\left|D^{k}(\mathbf{u})\right|(\Omega)<\infty .
$$

Conversely, if $\operatorname{Var}(\mathbf{u}, \Omega)<\infty$, then it follows that for any $\boldsymbol{\Phi} \in C_{c}^{1}\left(\Omega ; \mathbb{R}^{d \times d}\right)$,

$$
\left|\sum_{i, j} \int_{\Omega} L_{i, j}^{\mathrm{k}}(\mathbf{u})(\mathbf{x}) \cdot \nabla \boldsymbol{\Phi}_{i j}(\mathbf{x}) d \mathbf{x}\right| \leq \operatorname{Var}(\mathbf{u}, \Omega)\|\mathbf{\Phi}\|_{L^{\infty}} .
$$

Since $C_{c}^{1}\left(\Omega ; \mathbb{R}^{d \times d}\right)$ is dense in $C_{0}\left(\Omega ; \mathbb{R}^{d \times d}\right)$ in the standard norm of the latter, we can find a continuous linear functional $S$ on $C_{0}\left(\Omega ; \mathbb{R}^{d \times d}\right)$ such that for all $\boldsymbol{\Phi} \in C_{c}^{1}\left(\Omega ; \mathbb{R}^{d \times d}\right)$,

$$
S(\boldsymbol{\Phi})=\sum_{i, j} \int_{\Omega} L_{i, j}^{\mathrm{k}}(\mathbf{u})(\mathbf{x}) \cdot \nabla \boldsymbol{\Phi}_{i j}(\mathbf{x}) d \mathbf{x}
$$

and $\|S\| \leq \operatorname{Var}(\mathbf{u}, \Omega)$. By Riesz representation theorem, there exists an $\mathbb{R}^{d \times d}$ valued finite Radon matrix measure $\boldsymbol{\mu}=\left(\mu_{i j}\right)$ such that $\|S\|=|\boldsymbol{\mu}|(\Omega)$ and

$$
S(\boldsymbol{\Phi})=\sum_{i, j}^{d} \int_{\Omega} \boldsymbol{\Phi}_{i j}(\mathbf{x}) d \mu_{i j}, \quad \forall \boldsymbol{\Phi} \in C_{0}\left(\Omega ; \mathbb{R}^{d \times d}\right) .
$$

Using the action of $S$ on smooth test functions give in (3.6), we see that for all $\boldsymbol{\Phi} \in C_{c}^{1}\left(\Omega ; \mathbb{R}^{d \times d}\right)$,

$$
\sum_{i, j}^{d} \int_{\Omega} \boldsymbol{\Phi}_{i j}(\mathbf{x}) d \mu_{i j}=\sum_{i, j} \int_{\Omega} L_{i, j}^{\mathrm{k}}(\mathbf{u})(\mathbf{x}) \cdot \nabla \boldsymbol{\Phi}_{i j}(\mathbf{x}) d \mathbf{x},
$$

implying that for $\mathbf{u} \in \mathcal{M}_{k}$, we have $D^{\mathrm{K}}(\mathbf{u})=-\boldsymbol{\mu}$ and $\left|D^{\mathrm{K}}(\mathbf{u})\right|(\Omega)=\|S\|=\operatorname{Var}(\mathbf{u}, \Omega)$

Remark 3.10. There are other properties of the function space $\mathcal{M}_{\mathbf{k}}(\Omega)$ that one can prove using arguments from $B V$ and $B D$ spaces.

a) The map $\mathbf{u} \mapsto \operatorname{Var}(\mathbf{u}, \Omega)$ is lower semicontinuous in the sequential weak ${ }^{*}$ topology of $L_{\text {loc }}^{1}\left(\Omega ; \mathbb{R}^{d}\right)$. That is, if $\mathbf{u}_{n}, \mathbf{u} \in L_{\text {loc }}^{1}\left(\Omega ; \mathbb{R}^{d}\right)$ such that for any $\boldsymbol{\psi} \in C_{c}\left(\Omega ; \mathbb{R}^{d}\right)$

$$
\lim _{n \rightarrow \infty} \int_{\Omega} \mathbf{u}_{n} \cdot \boldsymbol{\psi} d \mathbf{x}=\int_{\Omega} \mathbf{u} \cdot \boldsymbol{\psi} d \mathbf{x}, \quad \text { then } \operatorname{Var}(\mathbf{u}, \Omega) \leq \liminf _{n \rightarrow \infty} \operatorname{Var}\left(\mathbf{u}_{n}, \Omega\right) .
$$

In fact, for each $\mathbf{\Phi} \in C_{c}^{1}\left(\Omega ; \mathbb{R}^{d \times d}\right)$

$$
\mathbf{u} \mapsto \sum_{i, j} \int_{\Omega} L_{i, j}^{\mathrm{k}}(\mathbf{u})(\mathbf{x}) \cdot \nabla \boldsymbol{\Phi}_{i j}(\mathbf{x}) d \mathbf{x}
$$

is continuous in the sequential weak* topology of $L_{\text {loc }}^{1}$. As a supremum, $\operatorname{Var}(\mathbf{u}, \Omega)$ will be lower semicontinuous. As a consequence, by Lemma 3.9 for any $\mathbf{u} \in \mathcal{M}_{\mathrm{k}}(\Omega)$, the map $\mathbf{u} \mapsto\left|D^{\mathrm{k}}(\mathbf{u})\right|(\Omega)$ is lower semicontinuous in the 
weak ${ }^{*}$ topology of $L_{l o c}^{1}\left(\Omega ; \mathbb{R}^{d}\right)$.

b) If $\mathbf{u} \in \mathcal{M}_{\mathbf{k}}(\Omega)$ and $A \subset \subset \Omega$, then for any $\eta \in C_{c}^{\infty}\left(\mathbb{R}^{d}\right)$ with $\operatorname{diam}(\operatorname{supt}(\eta))<\operatorname{dist}(A, \partial \Omega)$,

$$
\left|D^{\mathrm{k}}(\mathbf{u} * \eta)\right|(A) \leq\left(\int_{\mathbb{R}^{d}}|\eta| d \mathbf{x}\right)\left|D^{\mathrm{k}}(\mathbf{u})\right|(\Omega) .
$$

This is actually a consequence of Hölder's inequality preceded by the identity that for any $\phi \in C_{c}^{1}\left(A ; \mathbb{R}^{d}\right)$, and any $i, j=1,2, \cdots, d$,

$$
\int_{A} L_{i, j}^{\mathrm{k}}(\mathbf{u} * \eta)(\mathbf{x}) \cdot \nabla \phi(\mathbf{x}) d \mathbf{x}=\int_{\Omega}(\nabla \phi) * \eta(\mathbf{x}) \cdot L_{i, j}^{\mathrm{k}}(\mathbf{u}) d \mathbf{x}=\int_{\Omega} \nabla(\phi * \eta) \cdot L_{i, j}^{\mathrm{k}}(\mathbf{u}) d \mathbf{x} .
$$

As a result if $\eta^{\epsilon}$ is a standard sequence of mollifiers, then for all $\epsilon$,

$$
\left|D^{\mathrm{k}}\left(\mathbf{u} * \eta^{\epsilon}\right)\right|(A) \leq\left|D^{\mathrm{k}}(\mathbf{u})\right|(\Omega), \quad \text { and } \quad \lim _{\epsilon \rightarrow 0}\left|D^{\mathrm{k}}\left(\mathbf{u} * \eta^{\epsilon}\right)\right|(A) \leq\left|D^{\mathrm{k}}(\mathbf{u})\right|(\bar{A}) .
$$

In particular, using the lower semicontinuity property in a) and in the event $\left|D^{\mathrm{k}}(\mathbf{u})\right|(\partial A)=0$, we have

$$
\lim _{\epsilon \rightarrow 0}\left|D^{\mathrm{k}}\left(\mathbf{u} * \eta^{\epsilon}\right)\right|(A)=\left|D^{\mathrm{k}}(\mathbf{u})\right|(A) .
$$

c) Along the same line of argument as in the proof of density of smooth functions in BV spaces, the space of smooth functions $C^{\infty}\left(\Omega ; \mathbb{R}^{d}\right) \cap \mathcal{M}_{\mathrm{k}}(\Omega)$ is dense in the sense of strict convergence. That is, given $\mathbf{u} \in \mathcal{M}_{\mathrm{k}}(\Omega)$, there exists sequence $\mathbf{u}_{n} \in C^{\infty}\left(\Omega ; \mathbb{R}^{d}\right) \cap \mathcal{M}_{\mathbf{k}}(\Omega)$ such that

$$
\mathbf{u}_{n} \rightarrow \mathbf{u} \quad \text { strongly in } L^{1}\left(\Omega ; \mathbb{R}^{d}\right) \text { and }\left|D^{\mathrm{K}}\left(\mathbf{u}_{n}\right)\right|(\Omega) \rightarrow\left|D^{\mathrm{K}}(\mathbf{u})\right|(\Omega) \text { as } n \rightarrow \infty .
$$

See [5] for the proof this approximation result for BD function and [3] for BV functions.

d) In the above smooth approximation, for domains with smooth boundary it is known that $C^{\infty}\left(\Omega ; \mathbb{R}^{d}\right) \cap \mathcal{M}_{\mathrm{k}}(\Omega)$ can be replaced by $C^{\infty}\left(\bar{\Omega} ; \mathbb{R}^{d}\right)$ for both $B D(\Omega)$ and $B V(\Omega)$; see [3, Remark 3.22] for BV space and [36] for BD space.

Examples of third order tensor field and associated function spaces. For convenience, we introduce two positive constants. We define, for $\mathbf{z}=\left(z_{1}, z_{2}, \cdots, z_{d}\right)^{t} \in \mathbb{R}^{d}$,

$$
\begin{gathered}
\eta=f_{B(0,1)} \frac{z_{1}^{2}}{|\mathbf{z}|^{2}} d \mathbf{z}=f_{B(0,1)} \frac{z_{i}^{2}}{|\mathbf{z}|^{2}} d \mathbf{z}=\frac{3}{d(d+2)}, \quad \forall i, \\
\lambda=f_{B(0,1)} z_{1}^{2} z_{2}^{2} \frac{d \mathbf{z}}{|\mathbf{z}|^{4}}=f_{B(0,1)} z_{i}^{2} z_{j}^{2} \frac{d \mathbf{z}}{|\mathbf{z}|^{4}}=\frac{1}{d(d+2)}, \quad \forall i \neq j .
\end{gathered}
$$

For each of those tensor fields given in (1.3). we now identify the associated functions spaces in the same way as done in the previous section:

(1) For $\mathrm{K}_{1}(\boldsymbol{\xi})=\frac{\boldsymbol{\xi}}{|\boldsymbol{\xi}|} \otimes \mathbb{I}$, the action of the fourth order tensor $\mathrm{C}_{1}$ is given by

$$
\mathrm{C}_{1} \mathbb{A}^{T}=f_{B_{1}(0)} \mathrm{K}_{1}(\mathbf{z}) \mathbb{A} \frac{\mathbf{z}}{|\mathbf{z}|} d \mathbf{z}=\eta \mathbb{A}^{T} .
$$

In this case, $D^{\mathrm{k}_{1}}(\mathbf{u})=\eta \nabla \mathbf{u}^{t}$ and therefore the function spaces are precisely $\mathcal{W}_{\mathrm{k}_{1}}^{1, p}=W^{1, p}\left(\Omega ; \mathbb{R}^{d}\right)$ and $\mathcal{M}_{\mathrm{k}_{1}}(\Omega)=B V\left(\Omega ; \mathbb{R}^{d}\right)$.

(2) For $\mathrm{K}_{2}(\boldsymbol{\xi})=\frac{\boldsymbol{\xi}}{|\boldsymbol{\xi}|} \otimes \frac{\xi}{|\boldsymbol{\xi}|} \otimes \frac{\boldsymbol{\xi}}{|\boldsymbol{\xi}|}$, the action of the fourth order tensor $\mathrm{C}_{2} \mathbb{A}^{T}$ is given by

$$
\mathrm{C}_{2} \mathbb{A}^{T}=f_{B_{1}} \mathrm{~K}_{2}(\mathbf{z}) \mathbb{A} \frac{\mathbf{z}}{|\mathbf{z}|} d \mathbf{z}=f_{B_{1}}\langle\mathbb{A} \mathbf{z}, \mathbf{z}\rangle \mathbf{z} \otimes \mathbf{z} \frac{d \mathbf{z}}{|\mathbf{z}|^{4}}=\lambda\left(\mathbb{A}+\mathbb{A}^{T}\right)+\lambda \operatorname{Tr}(\mathbb{A}) \mathbb{I} .
$$

In this case $D^{\mathrm{k}_{2}}(\mathbf{u})=\lambda\left(\nabla \mathbf{u}+\nabla \mathbf{u}^{t}\right)+\lambda \operatorname{div} \mathbf{u} \mathbb{I}$, and

$$
\begin{aligned}
& \mathcal{W}_{\mathrm{k}_{2}}^{1, p}(\Omega)=\left\{\mathbf{u} \in L^{p}\left(\Omega ; \mathbb{R}^{d}\right):\left(\nabla \mathbf{u}+\nabla \mathbf{u}^{t}\right)+\operatorname{div}(\mathbf{u}) \mathbb{I} \in L^{p}\left(\Omega ; \mathbb{R}^{d \times d}\right)\right\} \\
& \mathcal{M}_{\mathrm{k}_{2}}(\Omega)=\left\{\mathbf{u} \in L^{1}\left(\Omega ; \mathbb{R}^{d}\right):\left(\nabla \mathbf{u}+\nabla \mathbf{u}^{t}\right)+\operatorname{div}(\mathbf{u}) \mathbb{I} \in M\left(\Omega ; \mathbb{R}^{d \times d}\right)\right\}
\end{aligned}
$$

Note that if $\mathbf{u}=\left(u_{1}, \cdots, u_{d}\right) \in \mathcal{W}_{\mathrm{k}_{2}}^{1, p}(\Omega)$, then for any $i, j \in\{1,2, \cdots, d\}, i \neq j$, the distribution $\partial_{i} u_{j}+\partial_{j} u_{i} \in L^{p}(\Omega)$. Moreover, for any $i$, we have $2 \partial_{i} u_{i}+\operatorname{div} \mathbf{u} \in L^{p}(\Omega)$. Adding over $i$, we obtain that $(d+2) \operatorname{div} \mathbf{u} \in L^{p}(\Omega)$. As a consequence, $2 \partial_{i} u_{i} \in L^{p}(\Omega)$ for all $i$. We conclude that if $\mathbf{u} \in \mathcal{W}_{\mathrm{k}_{2}}^{1, p}(\Omega)$, then its symmetric distributional gradient $\mathcal{E}(\mathbf{u})=\frac{\nabla \mathbf{u}+\nabla \mathbf{u}^{t}}{2}$ is in $L^{p}\left(\Omega ; \mathbb{R}^{d \times d}\right)$. It follows that if $1<p<\infty$, then Korn's inequality implies that the space $\mathcal{W}_{\mathrm{K}_{2}}^{1, p}(\Omega)$ is precisely $W^{1, p}\left(\Omega ; \mathbb{R}^{d}\right)$. 
Similarly if $\mathbf{u} \in \mathcal{M}_{\mathrm{k}_{2}}(\Omega)$, then $\mathcal{E}(\mathbf{u}) \in M\left(\Omega ; \mathbb{R}^{d \times d}\right)$ implying that $\mathcal{M}_{\mathrm{k}_{2}}(\Omega)$ is the space of functions of bounded deformation $B D\left(\Omega ; \mathbb{R}^{d}\right)$.

(3) To find the function space corresponding to $\mathrm{K}_{3}(\boldsymbol{\xi})=\left(\frac{\boldsymbol{\xi}}{|\boldsymbol{\xi}|} \otimes \frac{\boldsymbol{\xi}}{|\boldsymbol{\xi}|}-\frac{1}{3} \mathbb{I}\right) \otimes \frac{\boldsymbol{\xi}}{|\boldsymbol{\xi}|}$, we first note

$$
\mathrm{C}_{3} \mathbb{A}^{T}=\lambda\left(\mathbb{A}+\mathbb{A}^{T}\right)
$$

We see that $\mathcal{W}_{\mathrm{k}_{3}}^{1, p}(\Omega)=\left\{\mathbf{u} \in L^{p}\left(\Omega ; \mathbb{R}^{d}\right): \mathcal{E}(\mathbf{u}) \in L^{p}\left(\Omega ; \mathbb{R}^{d \times d}\right)\right\}$, where $2 \mathcal{E}(\mathbf{u})=\nabla \mathbf{u}+\nabla \mathbf{u}^{t}$ is the strain matrix, and therefore using Korn's inequality $\mathcal{W}_{\mathrm{k}_{3}}^{1, p}(\Omega)=W^{1, p}\left(\Omega ; \mathbb{R}^{d}\right)$. Also, $\mathcal{M}_{\mathrm{k}_{3}}(\Omega)=B D\left(\Omega ; \mathbb{R}^{d}\right)$.

(4) We now consider the case $\gamma=\frac{d \lambda+1}{2 d \eta}$, where $\lambda$ and $\eta$ are as defined above. If $\mathbf{K}_{4}(\boldsymbol{\xi})=\left(\frac{\boldsymbol{\xi}}{|\boldsymbol{\xi}|} \otimes \frac{\boldsymbol{\xi}}{|\boldsymbol{\xi}|}-\gamma \mathbb{I}\right) \otimes \frac{\boldsymbol{\xi}}{|\boldsymbol{\xi}|}$, then a simple calculation show that

$$
\begin{aligned}
& \mathcal{W}_{\mathrm{k}_{4}}^{1, p}\left(\Omega=\left\{\mathbf{u} \in L^{p}\left(\Omega ; \mathbb{R}^{d}\right): \mathcal{E}^{D}(\mathbf{u}) \in L^{p}\left(\Omega ; \mathbb{R}^{d \times d}\right)\right\},\right. \\
& \mathcal{M}_{\mathrm{k}_{4}}(\Omega)=\left\{\mathbf{u} \in L^{1}\left(\Omega ; \mathbb{R}^{d}\right): \mathcal{E}^{D}(\mathbf{u}) \in M\left(\Omega ; \mathbb{R}^{d \times d}\right)\right\},
\end{aligned}
$$

where $\mathcal{E}^{D}(\mathbf{u})$ is the deviatoric part of the strain given by $\mathcal{E}^{D}(\mathbf{u})=\mathcal{E}(\mathbf{u})-\frac{\mathbf{1}}{\mathbf{d}} \operatorname{div}(\mathbf{u}) \mathbb{I}$.

(5) Finally corresponding to $\mathrm{K}_{5}=\mathbb{I} \otimes \frac{\boldsymbol{\xi}}{|\boldsymbol{\xi}|}$, the function spaces are

$$
\begin{aligned}
& \mathcal{W}_{\mathrm{k}_{5}}^{1, p}(\Omega)=\left\{\mathbf{u} \in L^{p}\left(\Omega ; \mathbb{R}^{d}\right): \operatorname{div} \mathbf{u} \mathbb{I} \in L^{p}\left(\Omega ; \mathbb{R}^{d \times d}\right)\right\}, \\
& \mathcal{M}_{\mathrm{k}_{5}}(\Omega)=\left\{\mathbf{u} \in L^{1}\left(\Omega ; \mathbb{R}^{d}\right): \operatorname{div} \mathbf{u} \mathbb{I} \in M\left(\Omega ; \mathbb{R}^{d \times d}\right)\right\} .
\end{aligned}
$$

For $p=2$ the space $\mathcal{W}_{\mathrm{k}_{5}}^{1,2}(\Omega)$ can be identified with the vector space $H(\operatorname{div})(\Omega)$ that often is used in both continuum mechanics and electro-magnetics.

Remark 3.11. For $\mathrm{K}=\mathrm{K}_{i}$ where $i=1,2,3$ we remark that if both $\boldsymbol{\mu}$ and the $\mathrm{K}$-distributional derivative matrix $D^{\mathrm{k}}(\boldsymbol{\mu})$, as defined in Remark 3.7, are finite bounded Radon measures on $\Omega$, then $\boldsymbol{\mu}$ is represented by a function $\mathbf{u} \in L^{1}\left(\Omega ; \mathbb{R}^{d}\right)$. Indeed, in the case when $\mathrm{K}=\mathrm{K}_{1}$, then $\mathbf{u} \in B V\left(\Omega ; \mathbb{R}^{d}\right)$ and when $\mathrm{K}=\mathrm{K}_{i}, i=2,3$, then $\mathbf{u} \in B D(\Omega)$. The proofs of these statements can de obtained from the above calculation and together with $[3$, Exercise 3.2] for $B V$ case and in [5, Proposition 1.13] for the BD case.

Nonlocal characterization of spaces. Recall the distributional nonlocal gradient operator $\mathfrak{G}_{\rho_{n}}=\mathfrak{G}_{n}^{\mathrm{k}}$. For a given vector field $\mathbf{u}$ we would like to use the boundedness of $\mathfrak{G}_{n}^{\mathbf{k}} \mathbf{u}$ to characterize the function space that $\mathbf{u}$ belongs to.

Theorem 3.12. Suppose that $1 \leq p<\infty, \mathbf{u} \in L^{p}\left(\Omega ; \mathbb{R}^{d}\right)$ and $\mathfrak{G}_{n}^{\mathrm{k}} \mathbf{u}$ is a bounded sequence in $L^{p}\left(\Omega ; \mathbb{R}^{d \times d}\right)$, we have the following properties.

1. If $1<p<\infty$ then $\mathbf{u} \in \mathcal{W}_{\mathrm{k}}^{1, p}(\Omega)$ and $\mathfrak{G}_{n}^{\mathrm{k}} \mathbf{u}$ weakly converges to $D^{\mathrm{k}}(\mathbf{u})$ in $L^{p}\left(\Omega ; \mathbb{R}^{d \times d}\right)$.

2. If $p=1$, then $\mathbf{u} \in \mathcal{M}_{\mathrm{k}}(\Omega)$ and $\mathfrak{G}_{n}^{\mathrm{k}} \mathbf{u}$ weak ${ }^{*}$ converges to $D^{\mathrm{k}}(\mathbf{u})$.

Proof. Let $\mathbf{b} \in \mathbb{R}^{d}$ be arbitrary and $\psi \in C_{c}^{1}(\Omega)$. For $1<p<\infty$, up to a subsequence, not relabeled, let $\mathbb{V} \in L^{p}\left(\Omega ; \mathbb{R}^{d \times d}\right)$ be the weak limit of $\mathfrak{G}_{n}^{\mathrm{k}} \mathbf{u}$. Then by definition of the distribution $\mathfrak{G}_{n}^{\mathrm{k}} \mathbf{u}$, applying (3.4) and Corollary 3.5 we have

$$
\begin{aligned}
\int_{\Omega} \mathbb{V}(\mathbf{x}) \psi(\mathbf{x}) \mathbf{b} d \mathbf{x}=\lim _{n \rightarrow \infty} \int_{\Omega} \mathfrak{G}_{n}^{\mathrm{k}} \mathbf{u}(\mathbf{x}) \psi(\mathbf{x}) \mathbf{b} d \mathbf{x} & =-\lim _{n \rightarrow \infty} \int_{\Omega} \mathbb{D}_{n}^{+}(\psi \mathbf{b})(\mathbf{x}) \mathbf{u}(\mathbf{x}) d \mathbf{x} \\
& =-\int_{\Omega} f_{B_{1}(\mathbf{0})}(\mathrm{K}(\mathbf{z}) \mathbf{u}(\mathbf{x}) \otimes \mathbf{b}) \nabla \psi(\mathbf{x}) \cdot \frac{\mathbf{z}}{|\mathbf{z}|} d \mathbf{z}=\left\langle D^{\mathrm{K}}(\mathbf{u}) \mathbf{b}, \psi\right\rangle .
\end{aligned}
$$

where the last equality follows from the definition of the distribution $D^{\mathrm{K}}(\mathbf{u}) \mathbf{b}$. So for all $\mathbf{b} \in \mathbb{R}^{d}$, the function $\mathbb{V}(\mathbf{x}) \mathbf{b}$ agrees with the distribution $D^{\mathrm{K}}(\mathbf{u}) \mathbf{b}$, implying that $D^{\mathrm{K}}(\mathbf{u}) \in L^{p}\left(\Omega ; \mathbb{R}^{d \times d}\right)$. For $p=1$, we let the finite Radon measure $\boldsymbol{\mu}$ be the weak* limit of $\mathfrak{G}_{n}^{\mathrm{k}} \mathbf{u}$, By the above calculation and Lemma 3.9, we conclude that $\mathbf{u} \in \mathcal{M}_{\mathrm{k}}(\Omega)$ and that the distribution $D^{\mathrm{K}}(\mathbf{u})$ agrees with the finite measure $\boldsymbol{\mu}$ in the sense of distributions.

Remark 3.13. a). It follows from the weak lower semicontinuity of the norm and Corollary 3.5 that

$$
\left\|D^{\mathrm{K}}(\mathbf{u})\right\|_{L^{p}} \leq \liminf _{n \rightarrow \infty}\left\|\mathfrak{G}_{n}^{\mathrm{k}} \mathbf{u}\right\| \text { for } 1<p<\infty, \quad \text { and }\left|D^{\mathrm{K}}(\mathbf{u})\right|(\Omega) \leq \liminf _{n \rightarrow \infty}\left\|\mathfrak{G}_{n}^{\mathrm{k}} \mathbf{u}\right\|_{L^{1}} \text { for } p=1 \text {. }
$$

b). The theorem generalizes the nonlocal characterization of Sobolev, BV and BD function spaces found in literature $[9,31,25,29]$ and presents an alternative, perhaps simpler, proof. Namely that if $1 \leq p<\infty, \mathbf{u} \in L^{p}(\Omega)$ and

$$
\liminf _{n \rightarrow \infty} \int_{\Omega} \int_{\Omega} \rho_{n}(\mathbf{y}-\mathbf{x}) \frac{|\mathbf{u}(\mathbf{y})-\mathbf{u}(\mathbf{x})|^{p}}{|\mathbf{y}-\mathbf{x}|^{p}} d \mathbf{y} d \mathbf{x}<\infty,
$$


then $\mathbf{u} \in W^{1, p}\left(\Omega ; \mathbb{R}^{d}\right)$. This conclusion can be viewed as a corollary of Theorem 3.12. Indeed, in this case, using the integration by parts formula, Theorem 2.7, for any third order tensor field $\mathrm{K}$ satisfying (1.2), the $\mathrm{K}$-nonlocal gradient $\mathbb{G}_{n}^{\mathrm{k}} \mathbf{u}$ will coincide with the distributional nonlocal gradient $\mathfrak{G}_{n}^{\mathrm{k}} \mathbf{u}$. Moreover it follows from (3.7) that $\mathbb{G}_{n}^{\mathrm{k}} \mathbf{u}$ is bounded in $L^{p}\left(\Omega ; \mathbb{R}^{d \times d}\right)$ and therefore will converge weakly in $L^{p}\left(\Omega ; \mathbb{R}^{d \times d}\right)$ to $D^{\mathrm{k}}(\mathbf{u})$ by Theorem 3.12. In particular, if $\mathrm{K}(\boldsymbol{\xi})=\frac{\boldsymbol{\xi}}{|\boldsymbol{\xi}|} \otimes \mathbb{I}$, then $D^{\mathrm{k}}(\mathbf{u})=\eta \nabla \mathbf{u}^{t}$ and therefore $\mathbf{u} \in W^{1, p}\left(\Omega ; \mathbb{R}^{d}\right)$. Similar argument can be made when $p=1$ to show that $\mathbf{u} \in B V\left(\Omega ; \mathbb{R}^{d}\right)$. In [25] under a weaker condition, namely

$$
\liminf _{n \rightarrow \infty} \int_{\Omega} \int_{\Omega} \rho_{n}(\mathbf{y}-\mathbf{x}) \frac{|(\mathbf{y}-\mathbf{x}) \cdot(\mathbf{u}(\mathbf{y})-\mathbf{u}(\mathbf{x}))|^{p}}{|\mathbf{y}-\mathbf{x}|^{p+2}} d \mathbf{y} d \mathbf{x}<\infty
$$

it is shown that a vector field $\mathbf{u} \in L^{p}(\Omega)$ satisfying $(3.8)$ is in $W^{1, p}\left(\Omega ; \mathbb{R}^{d}\right)$ if $1<p<\infty$ and in $B D(\Omega)$ if $p=1$. Again this conclusion can be drawn from Theorem 3.12 by taking the third order tensor field $\mathbf{K}(\boldsymbol{\xi})=\frac{\boldsymbol{\xi}}{|\boldsymbol{\xi}|} \otimes \frac{\boldsymbol{\xi}}{|\boldsymbol{\xi}|} \otimes \frac{\boldsymbol{\xi}}{|\boldsymbol{\xi}|}$.

Theorem 3.12 generalizes the results in $[9,31,25,29]$ in the sense that the boundedness of the sequence $\mathfrak{G}_{n}^{\mathrm{k}}$ can be deduced from the weaker condition that $\rho_{n}^{1 / p}(\mathbf{y}-\mathbf{x})\left|\mathrm{K}(\mathbf{y}-\mathbf{x}) \mathcal{D}_{*} \mathbf{u}(\mathbf{y}, \mathbf{x})\right|$ is bounded in $L^{p}(\Omega \times \Omega)$, see Theorem 2.7.

Theorem 3.14. Suppose that $1 \leq p<\infty, \mathbf{u}_{n}$ is bounded in $L^{p}\left(\Omega ; \mathbb{R}^{d}\right)$ and $\mathfrak{G}_{n}^{\mathrm{k}} \mathbf{u}_{n}$ is a bounded sequence in $L^{p}\left(\Omega ; \mathbb{R}^{d \times d}\right)$. Then we have the following.

1. If $1<p<\infty$ and $\mathbf{u}$ is the weak limit of $\mathbf{u}_{n}$ in $L^{p}\left(\Omega ; \mathbb{R}^{d}\right)$, then $\mathbf{u} \in \mathcal{W}_{\mathrm{k}}^{1, p}(\Omega)$. Moreover, $\mathfrak{G}_{n}^{\mathrm{k}} \mathbf{u}_{n}$ weakly converges to $D^{\mathrm{K}}(\mathbf{u})$ in $L^{p}\left(\Omega ; \mathbb{R}^{d \times d}\right)$.

2. If $p=1, \mathbf{K}(\boldsymbol{\xi})=\frac{\boldsymbol{\xi}}{|\boldsymbol{\xi}|} \otimes \mathbb{I}$ and the finite Radon measure $\boldsymbol{\mu}$ is a weak ${ }^{*}$ limit of $\mathbf{u}_{n}$, then there exists $\mathbf{u} \in B V\left(\Omega ; \mathbb{R}^{d}\right)$ such that $\boldsymbol{\mu}=\mathbf{u} d \mathbf{x}$

3. If $p=1, \mathbf{K}(\boldsymbol{\xi})=\frac{\boldsymbol{\xi}}{|\boldsymbol{\xi}|} \otimes \frac{\boldsymbol{\xi}}{|\boldsymbol{\xi}|} \otimes \frac{\boldsymbol{\xi}}{|\boldsymbol{\xi}|}$ or $\mathbf{K}(\boldsymbol{\xi})=\left(\frac{\boldsymbol{\xi}}{|\boldsymbol{\xi}|} \otimes \frac{\boldsymbol{\xi}}{|\boldsymbol{\xi}|}-\frac{1}{3} \mathbb{I}\right) \otimes \frac{\boldsymbol{\xi}}{|\boldsymbol{\xi}|}$ and the finite Radon measure $\boldsymbol{\mu}$ is a weak* $^{*}$ limit of $\mathbf{u}_{n}$, then there exists $\mathbf{u} \in B D(\Omega)$ such that $\boldsymbol{\mu}=\mathbf{u} d \mathbf{x}$.

Proof. For part 1 , let $\mathbf{b} \in \mathbb{R}^{d}$ be arbitrary and $\psi \in C_{c}^{1}(\Omega)$. Up to a subsequence let $\mathbb{V} \in L^{p}\left(\Omega ; \mathbb{R}^{d \times d}\right)$ be the weak limit of $\mathfrak{G}_{n}^{\mathrm{k}} \mathbf{u}_{n}$. Then by definition

$$
\int_{\Omega} \mathbb{V}(\mathbf{x}) \psi(\mathbf{x}) \mathbf{b} d \mathbf{x}=\lim _{n \rightarrow \infty} \int_{\Omega} \mathfrak{G}_{n}^{\mathrm{k}} \mathbf{u}_{n}(\mathbf{x}) \psi(\mathbf{x}) \mathbf{b} d \mathbf{x}=-\lim _{n \rightarrow \infty} \int_{\Omega} \mathbb{D}_{n}^{+}(\psi \mathbf{b})(\mathbf{x}) \mathbf{u}_{n}(\mathbf{x}) d \mathbf{x}
$$

By Corollary $3.5, \mathbb{D}_{n}^{+}(\psi \mathbf{b})$ converges in $L^{p}\left(\Omega ; \mathbb{R}^{d}\right)$. Therefore since $\mathbf{u}_{n}$ weakly converges to $\mathbf{u}$ in $L^{p}\left(\Omega ; \mathbb{R}^{d}\right)$, we obtain after simplification that

$$
-\lim _{n \rightarrow \infty} \int_{\Omega} \mathbb{D}_{n}^{+}(\psi \mathbf{b})(\mathbf{x}) \mathbf{u}_{n}(\mathbf{x}) d \mathbf{x}=-\int_{\Omega} f_{B_{1}(\mathbf{0})}(\mathrm{K}(\mathbf{z}) \mathbf{u}(\mathbf{x}) \otimes \mathbf{b}) \nabla \psi(\mathbf{x}) \cdot \frac{\mathbf{z}}{|\mathbf{z}|} d \mathbf{z}=\left\langle D^{\mathrm{K}}(\mathbf{u}) \mathbf{b}, \psi\right\rangle .
$$

That is for all $\mathbf{b} \in \mathbb{R}^{d}, \mathbb{V}(\mathbf{x}) \mathbf{b}=D^{\mathrm{K}}(\mathbf{u}) \mathbf{b}$, implying that $D^{\mathrm{K}}(\mathbf{u})$ is in $L^{p}\left(\Omega ; \mathbb{R}^{d \times d}\right)$.

For parts 2 and 3 , we can prove in the same way. In these cases, any finite Radon measure that is the weak* limit of $\mathfrak{G}_{n}^{\mathrm{k}} \mathbf{u}_{n}$ will coincide with $D^{\mathrm{k}}(\boldsymbol{\mu})$ and by the Remark 3.11 we get that $\boldsymbol{\mu}$ is represented by a vector field that belongs to the respective function spaces.

\section{Nonlocal VARiational PROBLEMS}

Minimizing a nonlocal functional. In this section we use some of the analytical tools developed in previous sections to study a variational problem motivated by nonlocal continuum model of deformation of materials. Suppose a body occupying $\Omega \subset \mathbb{R}^{d}$ has undergone the deformation $\mathbf{y}(\mathbf{x})=\mathbf{x}+\mathbf{u}(\mathbf{x})$. The peridynamic model treats the body as a complex mass spring system. As such any two material points $\mathbf{x}$ and $\mathbf{x}^{\prime}$ are assumed to be connected by a bond $\boldsymbol{\xi}=\mathbf{x}^{\prime}-\mathbf{x}$. Under the uniform small strain theory [34], the strain of the bond $\mathbf{x}^{\prime}-\mathbf{x}$ is given by the nonlocal linearized strain

$$
\mathscr{D}(\mathbf{u})\left(\mathbf{x}^{\prime}, \mathbf{x}\right)=\mathcal{D}_{*}(\mathbf{u})\left(\mathbf{x}^{\prime}, \mathbf{x}\right) \cdot \frac{\mathbf{x}^{\prime}-\mathbf{x}}{\left|\mathbf{x}^{\prime}-\mathbf{x}\right|} .
$$

Part of this strain contributes to the dilatational (volume changing) component of the deformation and the remaining is the deviatoric component. In [33], it is suggested that for isotropic material, the dilatational (volume 
changing) component should account the collective deformation of bonds in a neighborhood of a material point $\mathbf{x}$ and is given by

$$
\mathscr{D}_{\text {dil }}(\mathbf{u})(\mathbf{x})=\frac{1}{d} \int_{\Omega} \rho\left(\left|\mathbf{x}^{\prime}-\mathbf{x}\right|\right) \mathscr{D}(\mathbf{u})\left(\mathbf{x}^{\prime}, \mathbf{x}\right) d \mathbf{x}^{\prime}
$$

where $\rho\left(\left|\mathbf{x}^{\prime}-\mathbf{x}\right|\right)$ measures the interaction strength of the bond between $\mathbf{x}^{\prime}$ and $\mathbf{x}$ is assumed to be nonnegative, radial and locally integrable. The nonlocal deviatoric part is then given by $\mathscr{D}_{\text {dev }}(\mathbf{u})\left(\mathbf{x}^{\prime}, \mathbf{x}\right)=\mathscr{D}(\mathbf{u})\left(\mathbf{x}^{\prime}, \mathbf{x}\right)-$ $\mathscr{D}_{\text {dil }}(\mathbf{u})(\mathbf{x})$. Following classical models (see [5] for example) one may write the strain energy of an elasto-plastic body subject to the Von Mises yield condition and Hencky's law as

$$
\int_{\Omega} \alpha(\mathbf{x}) \int_{\Omega} \rho\left(\mathbf{x}^{\prime}-\mathbf{x}\right) \psi\left(\mathscr{D}_{\operatorname{dev}}(\mathbf{u})\left(\mathbf{x}^{\prime}, \mathbf{x}\right)\right) d \mathbf{x}^{\prime} d \mathbf{x}+\int_{\Omega} \kappa(\mathbf{x})\left(\mathscr{D}_{\operatorname{dil}}(\mathbf{u})(\mathbf{x})\right)^{2} d \mathbf{x},
$$

where the functions $\alpha(\mathbf{x})$ and $\kappa(\mathbf{x})$ constitute material properties that are proportional to shear and bulk moduli of the material, and the function $\psi$ is given by

$$
\psi(t)= \begin{cases}\frac{1}{2}|t|^{2} & \text { if }|t| \leq 1 \\ |t|-\frac{1}{2} & \text { if }|t| \geq 1\end{cases}
$$

We note that the dependance of the shear and bulk moduli on $\mathbf{x}$ represent the heterogeneity of the material. In addition, the function $\psi$ is assumed to be a convex (continuous ) function that is quadratic near 0 and linear near infinity. Studying functionals of type (4.1) with their dominant term having a linear growth is not easy. Instead, we use the above model as motivation to study the minimization of energy functionals of the above type with their deviatoric strain energy having a $p$-growth at infinity for some $p>1$. In fact our goal is to study the energy $E_{\rho}(\mathbf{u})$ that is given by

$$
E_{\rho}(\mathbf{u})=\int_{\Omega} \kappa(\mathbf{x}) \Phi\left(\mathscr{D}_{\operatorname{dil}}(\mathbf{u})(\mathbf{x})\right) d \mathbf{x}+\int_{\Omega} \int_{\Omega} \alpha(\mathbf{x}) \rho\left(\mathbf{x}^{\prime}-\mathbf{x}\right) \Psi\left(\mathscr{D}_{\operatorname{dev}}(\mathbf{u})\left(\mathbf{x}^{\prime}, \mathbf{x}\right)\right) d \mathbf{x}^{\prime} d \mathbf{x}
$$

where $\Phi$ and $\Psi$ convex function with two different growth conditions at infinity. To study the variational problem we use the following notations and assumptions associated with the energy given in (4.2).

\section{Assumptions :}

A1) The functions $\kappa, \alpha: \Omega \rightarrow \mathbb{R}$ are in $L^{\infty}(\Omega)$ and that

$$
\inf _{\mathbf{x} \in \Omega} \kappa(\mathbf{x})=\kappa_{0}>0 ; \quad \inf _{\mathbf{x} \in \Omega} \alpha(\mathbf{x})=\alpha_{0}>0 .
$$

A2) The functions $\Phi$ and $\Psi$ are convex functions.

A3) For $1<p \leq q<\infty$ and positive constants $c$ and $C$, we have the growth condition of $\Phi$ and $\Psi$

$$
c\left(|t|^{q}-1\right) \leq \Phi(t) \leq C\left(|t|^{q}+1\right), \quad c\left(|t|^{p}-1\right) \leq \Psi(t) \leq C\left(|t|^{p}+1\right), \quad \text { for all } t \in \mathbb{R} .
$$

Note in particular that the functions $\Phi$ and $\Psi$ could be negative, however condition (4.3) implies that they are bounded from below. We denote their greatest lower bounds by $l_{\Phi}$ and $l_{\Psi}$ respectively. That is,

$$
\inf _{s \in \mathbb{R}} \Phi(s)=l_{\Phi}, \quad \inf _{s \in \mathbb{R}} \Psi(s)=l_{\Psi} .
$$

Over an admissible class of vector fields, that may be determined by imposing some (typically volumetric) constraints, the potential energy functional $E_{\rho}(\mathbf{u})-f(\mathbf{u})$ will be shown to attain its minimum, where $f(\mathbf{u})$ is the work done by a given external force, which is a functional defined on a proper subset of $L^{p}\left(\Omega ; \mathbb{R}^{d}\right)$.

First let us introduce the energy space given by

$$
\mathcal{S}_{\rho}^{p, q}(\Omega)=\left\{\mathbf{u} \in L^{p}\left(\Omega ; \mathbb{R}^{d}\right): E_{\rho}(\mathbf{u})<\infty\right\}
$$

Noting that $\mathscr{D}_{\text {dil }}(\mathbf{u})(\mathbf{x})$ is proportional to the trace of the nonlocal gradient matrix $\mathbb{G}_{\rho}^{\mathrm{k}_{2}} \mathbf{u}(\mathbf{x})$ (Indeed, $\mathscr{D}_{\text {dil }}(\mathbf{u})(\mathbf{x})=$ $\left.\frac{1}{d} \operatorname{Tr}\left[\mathbb{G}_{\rho}^{\mathrm{k}_{2}} \mathbf{u}(\mathbf{x})\right]\right)$ where $\mathrm{K}_{2}(\boldsymbol{\xi})=\frac{\boldsymbol{\xi}}{|\boldsymbol{\xi}|} \otimes \frac{\boldsymbol{\xi}}{|\boldsymbol{\xi}|} \otimes \frac{\boldsymbol{\xi}}{|\boldsymbol{\xi}|}$ as given in (1.3), we may see from the structure of the functional $E_{\rho}(\mathbf{u})$ and assumptions $\left.\left.\mathbf{A} 1\right)-\mathbf{A} 4\right)$, that

$$
\mathcal{S}_{\rho}^{p, q}(\Omega)=\left\{\mathbf{u} \in L^{p}\left(\Omega ; \mathbb{R}^{d}\right): \operatorname{Tr}\left[\mathbb{G}_{\rho}^{\mathrm{k}_{2}} \mathbf{u}\right] \in L^{q}(\Omega), \quad \rho^{1 / p}\left(\mathbf{x}^{\prime}-\mathbf{x}\right) \mathscr{D}(\mathbf{u})\left(\mathbf{x}^{\prime}, \mathbf{x}\right) \in L^{p}(\Omega \times \Omega)\right\}
$$

and that $\mathcal{S}_{\rho}^{p, q}(\Omega)$ is a Banach space with the norm

$$
\|\mid \mathbf{u}\|=\|\mathbf{u}\|_{L^{p}}+\left\|\operatorname{Tr}\left[\mathbb{G}_{\rho}^{\mathrm{k}_{2}} \mathbf{u}\right]\right\|_{L^{q}}+\left\|\rho^{1 / p}\left(\mathbf{x}^{\prime}-\mathbf{x}\right) \mathscr{D}(\mathbf{u})\left(\mathbf{x}^{\prime}, \mathbf{x}\right)\right\|_{L^{p}(\Omega \times \Omega)} .
$$


By defining the natural injective map $T: \mathcal{S}_{\rho}^{p, q}(\Omega) \rightarrow L^{p}(\Omega) \times L^{q}(\Omega) \times L^{p}(\Omega \times \Omega)$ given by

$$
T(\mathbf{u})=\left(\mathbf{u}, \operatorname{Tr}\left[\mathbb{G}_{\rho}^{\mathrm{k}_{2}} \mathbf{u}\right], \rho^{1 / p}\left(\mathbf{x}^{\prime}-\mathbf{x}\right) \mathscr{D}(\mathbf{u})\left(\mathbf{x}^{\prime}, \mathbf{x}\right)\right)
$$

it follows that $\mathcal{S}_{\rho}^{p, q}(\Omega)$ is a separable and reflexive Banach space. When $p=q$, we denote the space simply by $\mathcal{S}_{\rho}^{p}$ and this space has been studied in [28]. Note that one may consider

$$
|\mathbf{u}|_{\mathcal{S}_{\rho}}:=\left\|\rho^{1 / p}\left(\mathbf{x}^{\prime}-\mathbf{x}\right) \mathscr{D}(\mathbf{u})\left(\mathbf{x}^{\prime}, \mathbf{x}\right)\right\|_{L^{p}(\Omega \times \Omega)}
$$

as a semi-norm of $\mathbf{u}$ in $\mathcal{S}_{\rho}^{p}(\Omega)$ and that it vanishes if and only if $\mathbf{u}$ is an infinitesimal rigid displacement, that is, $\mathbf{u}(\mathbf{x})=\mathbb{Q} \mathbf{x}+\mathbf{c}$, where $\mathbb{Q}$ is a skew symmetric matrix, see [25, 28]. We denote this class by $\mathcal{R}$. The admissible class over which we would like to optimize the potential energy $E_{\rho}(\mathbf{u})-f(\mathbf{u})$ will certainly avoid nonzero members of $\mathcal{R}$. Let us specify the continuity and growth conditions of $f(\mathbf{u})$. We further assume that

A4) The function $f: L^{p}\left(\Omega ; \mathbb{R}^{d}\right) \rightarrow \mathbb{R}$ is continuous and its restriction $\left.f\right|_{\mathcal{S}_{\rho}^{p, q}}$ is weakly continuous with respect to the topology of $\mathcal{S}_{\rho}^{p, q}$. We also assume that there exist $r \in[0, p)$ and $C>0$ such that

$$
|f(\mathbf{u})| \leq C\left(1+\|\mathbf{u}\|_{L^{p}}^{r}\right), \quad \forall \mathbf{u} \in L^{p}\left(\Omega ; \mathbb{R}^{d}\right) .
$$

Given the growth conditions on $\Phi$ and $\Psi$, we have the following lemma which quantifies the growth of the functional $E_{\rho}(\mathbf{u})$ in terms of the seminorms of $\mathbf{u}$, thus giving us a coercivity estimate.

Lemma 4.1. Under assumptions A 1$)-\mathbf{A} 3)$, there exist positive constants $c$ and $C$ such that for any $\mathbf{u} \in \mathcal{S}_{\rho}^{p, q}(\Omega)$

$$
c\left(|\mathbf{u}|_{\mathcal{S}_{\rho}^{p}}^{p}+\left\|\operatorname{Tr}\left[\mathbb{G}_{\rho}^{\mathrm{k}_{2}} \mathbf{u}\right]\right\|_{L^{q}}^{q}\right)-\frac{1}{c} \leq E_{\rho}(\mathbf{u}) \leq C\left(|\mathbf{u}|_{\mathcal{S}_{\rho}^{p}}^{p}+\left\|\operatorname{Tr}\left[\mathbb{G}_{\rho}^{\mathrm{k}_{2}} \mathbf{u}\right]\right\|_{L^{q}}^{q}+1\right) .
$$

Proof. While the upper bound estimate is direct, the lower bound estimate in (4.5) is a combination of two estimates:

$$
E_{\rho}(\mathbf{u}) \geq C_{1}\left\|\operatorname{Tr}\left[\mathbb{G}_{\rho}^{\mathrm{k}_{2}} \mathbf{u}\right]\right\|_{L^{q}}^{q}-C_{2}, \quad \forall \mathbf{u} \in \mathcal{S}_{\rho}^{p, q}
$$

and

$$
E_{\rho}(\mathbf{u}) \geq C_{1}|\mathbf{u}|_{\mathcal{S}_{\rho}^{p}}^{p}-C_{2}, \quad \forall \mathbf{u} \in \mathcal{S}_{\rho}^{p, q} .
$$

Estimates (4.6) can be proved as follows. Since $\Psi$ bounded from below, $\Phi$ has $q$-growth at infinity and $\kappa(\mathbf{x}), \alpha(\mathbf{x})$ are bounded and positive, we have constants $C_{1}, C_{2}$ such that

$$
E_{\rho}(\mathbf{u}) \geq \int_{\Omega} \kappa(\mathbf{x}) \Phi\left(\mathscr{D}_{\text {dil }}(\mathbf{u})(\mathbf{x})\right) d \mathbf{x}-\alpha_{0}\|\rho\|_{L^{1}} l_{\Psi} \geq C_{1} \int_{\Omega} \mid\left(\left.\mathscr{D}_{\operatorname{dil}}(\mathbf{u})(\mathbf{x})\right|^{q} d \mathbf{x}-C_{2}\right.
$$

from which (4.6) follows. Let us prove estimate (4.7). From assumption A1)- A3) we obtain positive constants $C_{1}, C_{2}$ such that

$$
E_{\rho}(\mathbf{u}) \geq C_{1}\left(\int_{\Omega} \int_{\Omega} \rho\left(\mathbf{x}^{\prime}-\mathbf{x}\right)\left|\mathscr{D}(\mathbf{u})\left(\mathbf{x}^{\prime}, \mathbf{x}\right)-\frac{1}{d} \operatorname{Tr}\left[\mathbb{G}_{\rho}^{\mathrm{k}_{2}} \mathbf{u}\right](\mathbf{x})\right|^{p} d \mathbf{x}^{\prime} d \mathbf{x}+\left\|\operatorname{Tr}\left[\mathbb{G}_{\rho}^{\mathrm{k}_{2}} \mathbf{u}\right]\right\|_{L^{q}}^{q}\right)-C_{2} .
$$

Using the inequality that $|t|^{p} \geq 2^{1-p}|t+s|^{p}-|s|^{p}$ for any $t, s$, it follows from the above inequality that there are positive generic constants $C_{1}$ and $C_{2}$ such that for any $\mathbf{u} \in \mathcal{S}_{\rho}^{p, q}(\Omega)$,

$$
\begin{aligned}
E_{\rho}(\mathbf{u}) & \geq C_{1}\left(|\mathbf{u}|_{\mathcal{S}_{\rho}^{p}}^{p}+\left\|\operatorname{Tr}\left[\mathbb{G}_{\rho}^{\mathrm{k}_{2}} \mathbf{u}\right]\right\|_{L^{q}}^{q}\right)-C_{2}\left(\left\|\operatorname{Tr}\left[\mathbb{G}_{\rho}^{\mathrm{k}_{2}} \mathbf{u}\right]\right\|_{L^{p}}^{p}+1\right) \\
& =C_{1}|\mathbf{u}|_{\mathcal{S}_{\rho}^{p}}^{p}+C_{1}\left\|\operatorname{Tr}\left[\mathbb{G}_{\rho}^{\mathrm{k}_{2}} \mathbf{u}\right]\right\|_{L^{q}}^{q}-C_{2}\left\|\operatorname{Tr}\left[\mathbb{G}_{\rho}^{\mathrm{k}_{2}} \mathbf{u}\right]\right\|_{L^{q}}^{p}-C_{2}
\end{aligned}
$$

where we have applied Hölder's inequality using the assumption that $p \leq q$. We separate two cases. The first case is when $q>p$. In this case, noting that the function $\tau \mapsto C_{1}|\tau|^{q}-C_{2}|\tau|^{p}$ is bounded from below in $\mathbb{R}$, we conclude that the difference $C_{1}\left\|\operatorname{Tr}\left[\mathbb{G}_{\rho}^{\mathrm{k}_{2}} \mathbf{u}\right]\right\|_{L^{q}}^{q}-C_{2}\left\|\operatorname{Tr}\left[\mathbb{G}_{\rho}^{\mathrm{k}_{2}} \mathbf{u}\right]\right\|_{L^{p}}^{p}$ is bounded from below uniformly for any u $\in \mathcal{S}_{\rho}^{p, q}(\Omega)$, implying estimate (4.7).

The second case is $p=q$, in this case we have that for all $\mathbf{u} \in \mathcal{S}_{\rho}^{p, q}(\Omega)$,

$$
C_{2}\left\|\operatorname{Tr}\left[\mathbb{G}_{\rho}^{k_{2}} \mathbf{u}\right]\right\|_{L^{p}}^{p}+E_{\rho}(\mathbf{u}) \geq C_{1}|\mathbf{u}|_{\mathcal{S}_{\rho}^{p}}^{p}-C_{2} .
$$

Apply (4.6) to estimate $\left\|\operatorname{Tr}\left[\mathbb{G}_{\rho}^{\mathrm{k}_{2}} \mathbf{u}\right]\right\|_{L^{p}}^{p}$ from above. That demonstrates (4.7).

Another important estimate is given below which will be used to show that $E_{\rho}$ is weakly lower semicontinuous. 
Lemma 4.2. Let $\Omega^{0} \subset \subset \Omega$ and $\epsilon_{0}=\min \left\{\operatorname{dist}(A, \Omega \backslash B)\right.$, dist $\left.\left(\Omega^{0}, \partial \Omega\right)\right\}$ for two subsets $A$ and $B$ of $\Omega$ such that $A \subset \subset B \subset \Omega$. Given $\phi_{\epsilon}$ a standard sequence of mollifiers, and $\mathbf{u} \in L^{p}\left(\Omega ; \mathbb{R}^{d}\right)$, define $\mathbf{u}_{\epsilon}:=\mathbf{u} * \phi_{\epsilon}$ for all $\epsilon<\epsilon_{0}$. Under assumptions $\mathbf{A} 2$ ) and $\mathbf{A} 3)$, we have that for all $\epsilon<\epsilon_{0}$

$$
\begin{aligned}
\int_{A} \int_{\Omega^{0}} \rho\left(\mathbf{x}^{\prime}-\mathbf{x}\right) & \Psi\left(\mathscr{D}\left(\mathbf{u}_{\epsilon}\right)\left(\mathbf{x}^{\prime}, \mathbf{x}\right)-\frac{1}{d} \phi_{\epsilon} * \operatorname{Tr}\left[\mathbb{G}_{\rho}^{\mathrm{k}_{2}} \mathbf{u}\right](\mathbf{x})\right) d \mathbf{x}^{\prime} d \mathbf{x} \\
& \leq \int_{B} \int_{\Omega} \rho\left(\mathbf{x}^{\prime}-\mathbf{x}\right) \Psi\left(\mathscr{D}(\mathbf{u})\left(\mathbf{x}^{\prime}, \mathbf{x}\right)-\frac{1}{d} \operatorname{Tr}\left[\mathbb{G}_{\rho}^{\mathrm{k}_{2}} \mathbf{u}\right](\mathbf{x})\right) d \mathbf{x}^{\prime} d \mathbf{x} \\
& +l_{\Psi}\left[\int_{A} \int_{\Omega^{0}} \rho\left(\mathbf{x}^{\prime}-\mathbf{x}\right) d \mathbf{x}^{\prime} d \mathbf{x}-\int_{B} \int_{\Omega} \rho\left(\mathbf{x}^{\prime}-\mathbf{x}\right) d \mathbf{x}^{\prime} d \mathbf{x}\right]
\end{aligned}
$$

Proof. It is enough to show the inequality for $\Psi \geq 0$, i. e. $l_{\Psi}=0$. The general estimate in (4.8) can be obtained by applying the proven result on $\Psi-l_{\Psi}$. Let us estimate as follows. First let us rewrite the convolution explicitly

$$
\begin{aligned}
& \int_{A} \int_{\Omega^{0}} \rho\left(\mathbf{x}^{\prime}-\mathbf{x}\right) \Psi\left(\mathscr{D}\left(\phi_{\epsilon} * \mathbf{u}\right)\left(\mathbf{x}^{\prime}, \mathbf{x}\right)-\gamma \phi_{\epsilon} * \operatorname{Tr}\left[\mathbb{G}_{\rho}^{\mathrm{k}_{2}} \mathbf{u}\right](\mathbf{x})\right) d \mathbf{x}^{\prime} d \mathbf{x} \\
& =\int_{A} \int_{\Omega^{0}} \rho\left(\mathbf{x}^{\prime}-\mathbf{x}\right) \Psi\left(\int_{B_{\epsilon}(\mathbf{0})}\left\{\mathscr{D}(\mathbf{u})\left(\mathbf{x}^{\prime}-\mathbf{z}, \mathbf{x}-\mathbf{z}\right)-\gamma \operatorname{Tr}\left[\mathbb{G}_{\rho}^{\mathrm{k}_{2}} \mathbf{u}\right](\mathbf{x}-\mathbf{z})\right\} \phi_{\epsilon}(\mathbf{z}) d \mathbf{z}\right) d \mathbf{x}^{\prime} d \mathbf{x} .
\end{aligned}
$$

For each fixed $\mathbf{x}^{\prime}, \mathbf{x}$, we apply Jensen's inequality using the fact that $\Psi$ is a convex and $\left\|\phi_{\epsilon}\right\|_{B_{\epsilon}(\mathbf{0})}=1$. We then obtain the inequality that

$$
\begin{aligned}
& \int_{A} \int_{\Omega^{0}} \rho\left(\mathbf{x}^{\prime}-\mathbf{x}\right) \Psi\left(\mathscr{D}\left(\phi_{\epsilon} * \mathbf{u}\right)\left(\mathbf{x}^{\prime}, \mathbf{x}\right)-\gamma \phi_{\epsilon} * \operatorname{Tr}\left[\mathbb{G}_{\rho}^{\mathrm{k}_{2}} \mathbf{u}\right](\mathbf{x})\right) d \mathbf{x}^{\prime} d \mathbf{x} \\
& \leq \int_{B_{\epsilon}(\mathbf{0})} \phi_{\epsilon}(\mathbf{z}) \int_{A} \int_{\Omega^{0}} \rho\left(\mathbf{x}^{\prime}-\mathbf{x}\right) \Psi\left(\left\{\mathscr{D}(\mathbf{u})\left(\mathbf{x}^{\prime}-\mathbf{z}, \mathbf{x}-\mathbf{z}\right)-\gamma \operatorname{Tr}\left[\mathbb{G}_{\rho}^{\mathrm{k}_{2}} \mathbf{u}\right](\mathbf{x}-\mathbf{z})\right\}\right) d \mathbf{x}^{\prime} d \mathbf{x} d \mathbf{z} .
\end{aligned}
$$

Noting that for $\mathbf{z} \in B_{\epsilon}(\mathbf{0})$ and $\epsilon<\epsilon_{0}, A-\mathbf{z} \subset B$ and $\Omega^{0}-\mathbf{z} \in \Omega$, we make a change of variables $\mathbf{x} \leftarrow \mathbf{x}-\mathbf{z}$ and $\mathbf{x}^{\prime} \leftarrow \mathbf{x}^{\prime}-\mathbf{z}$ and obtain that

$$
\begin{aligned}
& \int_{B_{\epsilon}(\mathbf{0})} \phi_{\epsilon}(\mathbf{z}) \int_{A} \int_{A} \rho\left(\mathbf{x}^{\prime}-\mathbf{x}\right) \Psi\left(\left\{\mathscr{D}(\mathbf{u})\left(\mathbf{x}^{\prime}-\mathbf{z}, \mathbf{x}-\mathbf{z}\right)-\gamma \operatorname{Tr}\left[\mathbb{G}_{\rho}^{\mathrm{k}_{2}} \mathbf{u}\right](\mathbf{x}-\mathbf{z})\right\}\right) d \mathbf{x}^{\prime} d \mathbf{x} d \mathbf{z} \\
& =\int_{B_{\epsilon}(\mathbf{0})} \phi_{\epsilon}(\mathbf{z}) \int_{A-\mathbf{z}} \int_{\Omega^{0}-\mathbf{z}} \rho\left(\mathbf{x}^{\prime}-\mathbf{x}\right) \Psi\left(\left\{\mathscr{D}(\mathbf{u})\left(\mathbf{x}^{\prime}, \mathbf{x}\right)-\gamma \operatorname{Tr}\left[\mathbb{G}_{\rho}^{\mathrm{k}_{2}} \mathbf{u}\right](\mathbf{x})\right\}\right) d \mathbf{x}^{\prime} d \mathbf{x} d \mathbf{z} \\
& \leq \int_{B} \int_{\Omega} \rho\left(\mathbf{x}^{\prime}-\mathbf{x}\right) \Psi\left(\left\{\mathscr{D}(\mathbf{u})\left(\mathbf{x}^{\prime}, \mathbf{x}\right)-\gamma \operatorname{Tr}\left[\mathbb{G}_{\rho}^{\mathrm{k}_{2}} \mathbf{u}\right](\mathbf{x})\right\}\right) d \mathbf{x}^{\prime} d \mathbf{x}
\end{aligned}
$$

where in the last inequality we used the fact that $\left\|\phi_{\epsilon}\right\|_{B_{\epsilon}(\mathbf{0})}=1$. Combining the last two sets of inequalities, we complete the proof of the lemma.

Last, we state without proof a nonlocal Poincaré-type inequality that will be used in the sequel. For the proof, see $[28]$.

Proposition 4.3 (Poincaré-type inequality). Suppose that $V \subset L^{p}\left(\Omega ; \mathbb{R}^{d}\right)$ is a weakly closed subset of $L^{p}\left(\Omega ; \mathbb{R}^{d}\right)$ that does not include nontrivial infinitesimal rigid displacements. Then there exists a constant $C=C(V, \rho)$ such that

$$
\|\mathbf{u}\|_{L^{p}}^{p} \leq C \int_{\Omega} \int_{\Omega} \rho(\mathbf{y}-\mathbf{x})\left|\mathscr{D}(\mathbf{u})\left(\mathbf{x}^{\prime}, \mathbf{x}\right)\right|^{p} d \mathbf{x}^{\prime} d \mathbf{x}, \quad \forall \mathbf{u} \in V .
$$

We are now ready to state and prove the main result of the section on the existence of solution to the nonlocal variational problem stated above.

Theorem 4.4. Suppose that $V \subset L^{p}\left(\Omega ; \mathbb{R}^{d}\right)$ is a weakly closed subset of $L^{p}\left(\Omega ; \mathbb{R}^{d}\right)$ that does not include nontrivial infinitesimal rigid displacements. Under assumptions $\mathbf{A} 1)-\mathbf{A} 4)$, the variational problem

$$
\inf _{\mathbf{u} \in V}\left\{E_{\rho}(\mathbf{u})-f(\mathbf{u})\right\}
$$

has a minimizer $\mathbf{u} \in V \cap \mathcal{S}_{\rho}^{p, q}(\Omega)$. 
Proof. As we mentioned earlier, the proof uses the direct method of calculus of variations. We need to show two things: the functional $E_{\rho}(\mathbf{u})-f(\mathbf{u})$ is coercive on $V \cap \mathcal{S}_{\rho}^{p, q}(\Omega)$ and is weakly lower semicontinous on $\mathcal{S}_{\rho}^{p, q}(\Omega)$. The coercivity of the functional follows from Lemma 4.1 and Proposition 4.3. Indeed, from Lemma 4.1 and assumption A4) we have the estimate that

$$
E_{\rho}(\mathbf{u}) \geq c\left(|\mathbf{u}|_{\mathcal{S}_{\rho}^{p}}^{p}+\left\|\operatorname{Tr}\left[\mathbb{G}_{\rho}^{\mathrm{k}_{2}} \mathbf{u}\right]\right\|_{L^{q}}^{q}\right)-\frac{1}{c}\left(\|\mathbf{u}\|_{L^{p}}^{r}+1\right)
$$

for some universal positive constant $c$ and all $\mathbf{u} \in \mathcal{S}_{\rho}^{p, q}(\Omega)$. Applying the nonlocal Poincaré-type inequality Proposition 4.3 we obtain that for some constant $C=C(V)$, and all $\mathbf{u} \in V \cap \mathcal{S}_{\rho}^{p, q}(\Omega)$

$$
|\mathbf{u}|_{\mathcal{S}_{\rho}^{p}}^{p} \geq C\left(|\mathbf{u}|_{\mathcal{S}_{\rho}^{p}}^{p}+\|\mathbf{u}\|_{L^{p}}^{p}\right) .
$$

The coercivity of the functional, then, follows combining the above inequalities.

Let us next show that the functional is weakly lower semicontinuous. To do that, let $\mathbf{u}_{n} \rightarrow \mathbf{u}$ in $\mathcal{S}_{\rho}^{p, q}(\Omega)$. Then necessarly $\mathbf{u}_{n} \rightarrow \mathbf{u}$ in $\mathcal{S}_{\rho}^{p}(\Omega)$, since $\mathcal{S}_{\rho}^{p, q}(\Omega) \subset \mathcal{S}_{\rho}^{p}(\Omega)$. Moreover, the sequence $\operatorname{Tr}\left[\mathbb{G}_{\rho}^{\mathrm{k}_{2}} \mathbf{u}_{n}\right]$ is bounded in $L^{q}(\Omega)$. From $\mathbf{u}_{n} \rightarrow \mathbf{u}$ in $\mathcal{S}_{\rho}^{p}(\Omega)$ we also have that $\operatorname{Tr}\left[\mathbb{G}_{\rho}^{\mathrm{k}_{2}} \mathbf{u}_{n}\right]$ weakly converges to $\operatorname{Tr}\left[\mathbb{G}_{\rho}^{\mathrm{k}_{2}} \mathbf{u}\right]$ in $L^{p}(\Omega)$ because the operator $\operatorname{Tr}\left[\mathbb{G}_{\rho}^{\mathrm{k}_{2}}\right]: \mathcal{S}_{\rho}^{p}(\Omega) \rightarrow L^{p}(\Omega)$ is a bounded linear operator $\left[12\right.$, Theorem 3.10]. Now $\operatorname{Tr}\left[\mathbb{G}_{\rho}^{\mathrm{k}_{2}} \mathbf{u}_{n}\right]$ is bounded in $L^{q}(\Omega)$ implying that, up to a subsequence, it weakly converges in $L^{q}(\Omega)$. Moreover, any weak limit must coincide with $\operatorname{Tr}\left[\mathbb{G}_{\rho}^{\mathrm{k}_{2}} \mathbf{u}\right]$, implying that the whole sequence $\operatorname{Tr}\left[\mathbb{G}_{\rho}^{\mathrm{k}_{2}} \mathbf{u}_{n}\right]$ weakly converges to $\operatorname{Tr}\left[\mathbb{G}_{\rho}^{\mathrm{k}_{2}} \mathbf{u}\right]$ in $L^{q}(\Omega)$. Now from the growth condition and convexity of $\Phi$ and the boundedness assumption on $\kappa(\mathbf{x})$, the map

$$
\mathbf{v} \mapsto \int_{\Omega} \kappa(\mathbf{x}) \Phi(\mathbf{v}(\mathbf{x})) d \mathbf{x}
$$

is weakly lower semicontinuous in $L^{q}(\Omega)$ (see [21]) and therefore,

$$
\int_{\Omega} \kappa(\mathbf{x}) \Phi\left(\mathscr{D}_{\text {dil }}(\mathbf{u})(\mathbf{x})\right) d \mathbf{x} \leq \liminf _{n \rightarrow \infty} \int_{\Omega} \kappa(\mathbf{x}) \Phi\left(\mathscr{D}_{\operatorname{dil}}\left(\mathbf{u}_{n}\right)(\mathbf{x})\right) d \mathbf{x}
$$

We now show the lower semicontinuity of the second part. We will show the inequality in three steps. First we show a limit inequality when $\alpha \equiv 1$ and over any subset $B$ of $\Omega$. Then in the second step we show the lower semicontinuity inequality for $\alpha$ any positive simple function. In the final step we approximate any $\alpha \in L^{\infty}(\Omega)$ by an increasing sequence of simple functions to prove lower semicontinuity. To begin, let $\mathbf{u}_{n} \rightarrow \mathbf{u}$ in $\mathcal{S}_{\rho}^{p, q}(\Omega)$.

Step 1. Suppose that $B \subset \Omega$. In this step we show that

$$
\begin{aligned}
\int_{B} \int_{\Omega} \rho\left(\mathbf{x}^{\prime}-\mathbf{x}\right) \Psi & \left.\left(\mathscr{D}(\mathbf{u})\left(\mathbf{x}^{\prime}, \mathbf{x}\right)-\gamma \operatorname{Tr}\left[\mathbb{G}_{\rho}^{\mathrm{k}_{2}} \mathbf{u}(\mathbf{x})\right]\right)\right) d \mathbf{x}^{\prime} d \mathbf{x} \\
\leq & \left.\liminf _{n \rightarrow \infty} \int_{B} \int_{\Omega} \rho\left(\mathbf{x}^{\prime}-\mathbf{x}\right) \Psi\left(\mathscr{D}\left(\mathbf{u}_{n}\right)\left(\mathbf{x}^{\prime}, \mathbf{x}\right)-\gamma \operatorname{Tr}\left[\mathbb{G}_{\rho}^{\mathrm{k}_{2}} \mathbf{u}_{n}(\mathbf{x})\right]\right)\right) d \mathbf{x}^{\prime} d \mathbf{x} .
\end{aligned}
$$

To that end, let $A \subset \subset B$ and $\Omega^{0} \subset \subset \Omega$ and $\epsilon_{0}=\min \left\{\operatorname{dist}(A, \Omega \backslash B),\left\{\operatorname{dist}\left(\Omega^{0}, \partial \Omega\right)\right\}>0\right.$. For any $\epsilon<\epsilon_{0}$ and a given sequence of standard mollifiers $\phi_{\epsilon}$, let us introduce the vector functions $\phi_{\epsilon} * \mathbf{u}_{n}$ is defined on $\Omega_{\epsilon}$. Applying Lemma 4.2 we obtain that for all $n \in \mathbb{N}$ and $\epsilon<\epsilon_{0}$,

$$
\begin{aligned}
\int_{A} \int_{\Omega^{0}} \rho\left(\mathbf{x}^{\prime}-\mathbf{x}\right) \Psi & \left(\mathscr{D}\left(\phi_{\epsilon} * \mathbf{u}_{n}\right)\left(\mathbf{x}^{\prime}, \mathbf{x}\right)-\frac{1}{d} \phi_{\epsilon} * \operatorname{Tr}\left[\mathbb{G}_{\rho}^{\mathrm{k}_{2}} \mathbf{u}_{n}\right](\mathbf{x})\right) d \mathbf{x}^{\prime} d \mathbf{x} \\
& \leq \int_{B} \int_{\Omega} \rho\left(\mathbf{x}^{\prime}-\mathbf{x}\right) \Psi\left(\mathscr{D}\left(\mathbf{u}_{n}\right)\left(\mathbf{x}^{\prime}, \mathbf{x}\right)-\frac{1}{d} \operatorname{Tr}\left[\mathbb{G}_{\rho}^{\mathrm{k}_{2}} \mathbf{u}_{n}\right](\mathbf{x})\right) d \mathbf{x}^{\prime} d \mathbf{x} \\
& +l_{\Psi}\left[\int_{A} \int_{\Omega^{0}} \rho\left(\mathbf{x}^{\prime}-\mathbf{x}\right) d \mathbf{x}^{\prime} d \mathbf{x}-\int_{B} \int_{\Omega} \rho\left(\mathbf{x}^{\prime}-\mathbf{x}\right) d \mathbf{x}^{\prime} d \mathbf{x}\right]
\end{aligned}
$$

Our next plan is to fix $\epsilon$ and take the liminf as $n \rightarrow \infty$ on both sides of the inequality. To do that we first note that for fixed $\epsilon$, the sequence of functions $\left\{\phi_{\epsilon} * \mathbf{u}_{n}\right\}_{n}$ is pre-compact in $L^{p}\left(\Omega_{\epsilon} ; \mathbb{R}^{d}\right)$, see [12, Corollary 4.28]. Moreover, since $\mathbf{u}_{n}$ weakly converges to $\mathbf{u}$, every subsequence of $\phi_{\epsilon} * \mathbf{u}_{n}$ will have a convergent subsubsequence in $L^{p}\left(\Omega_{\epsilon} ; \mathbb{R}^{d}\right)$ with the limit being $\phi_{\epsilon} * \mathbf{u}$. Therefore, the whole sequence $\phi_{\epsilon} * \mathbf{u}_{n}$ must converge to $\phi_{\epsilon} * \mathbf{u}$ in $L^{p}\left(\Omega_{\epsilon} ; \mathbb{R}^{d}\right)$ as $n \rightarrow \infty$. Similarly as $n \rightarrow \infty, \phi_{\epsilon} * \operatorname{Tr}\left[\mathbb{G}_{\rho}^{\mathrm{k}_{2}} \mathbf{u}_{n}(\mathbf{x})\right]$ strongly converges to $\phi_{\epsilon} * \operatorname{Tr}\left[\mathbb{G}_{\rho}^{\mathrm{k}_{2}} \mathbf{u}(\mathbf{x})\right]$ in $L^{p}(A)$. With these information at hand, we now take the liminf in $n$ on both sides of the inequality (4.10) and apply Fatou's lemma 
on the left hand side to obtain that for any fixed $\epsilon$,

$$
\begin{aligned}
\int_{A} \int_{\Omega^{0}} \rho\left(\mathbf{x}^{\prime}-\mathbf{x}\right) \Psi & \left.\left(\mathscr{D}\left(\phi_{\epsilon} * \mathbf{u}\right)\left(\mathbf{x}^{\prime}, \mathbf{x}\right)-\gamma \phi_{\epsilon} * \operatorname{Tr}\left[\mathbb{G}_{\rho}^{\mathrm{k}_{2}} \mathbf{u}(\mathbf{x})\right]\right)\right) d \mathbf{x}^{\prime} d \mathbf{x} \\
\leq & \left.\liminf _{n \rightarrow \infty} \int_{B} \int_{\Omega} \rho\left(\mathbf{x}^{\prime}-\mathbf{x}\right) \Psi\left(\mathscr{D}\left(\mathbf{u}_{n}\right)\left(\mathbf{x}^{\prime}, \mathbf{x}\right)-\gamma \operatorname{Tr}\left[\mathbb{G}_{\rho}^{\mathrm{k}_{2}} \mathbf{u}_{n}(\mathbf{x})\right]\right)\right) d \mathbf{x}^{\prime} d \mathbf{x} \\
& +l_{\Psi}\left[\int_{A} \int_{\Omega^{0}} \rho\left(\mathbf{x}^{\prime}-\mathbf{x}\right) d \mathbf{x}^{\prime} d \mathbf{x}-\int_{B} \int_{\Omega} \rho\left(\mathbf{x}^{\prime}-\mathbf{x}\right) d \mathbf{x}^{\prime} d \mathbf{x}\right]
\end{aligned}
$$

Now use the fact that as $\epsilon \rightarrow 0, \mathscr{D}\left(\phi_{\epsilon} * \mathbf{u}\right)\left(\mathbf{x}^{\prime}, \mathbf{x}\right) \rightarrow \mathscr{D}(\mathbf{u})\left(\mathbf{x}^{\prime}, \mathbf{x}\right)$ for almost every $\left(\mathbf{x}^{\prime}, \mathbf{x}\right) \in \Omega^{0} \times A$ and apply Fatou's lemma again to obtain that

$$
\begin{aligned}
\int_{A} \int_{\Omega^{0}} \rho\left(\mathbf{x}^{\prime}-\mathbf{x}\right) \Psi & \left.\left(\mathscr{D}(\mathbf{u})\left(\mathbf{x}^{\prime}, \mathbf{x}\right)-\gamma \operatorname{Tr}\left[\mathbb{G}_{\rho}^{\mathrm{k}_{2}} \mathbf{u}(\mathbf{x})\right]\right)\right) d \mathbf{x}^{\prime} d \mathbf{x} \\
\leq & \left.\liminf _{n \rightarrow \infty} \int_{B} \int_{\Omega} \rho\left(\mathbf{x}^{\prime}-\mathbf{x}\right) \Psi\left(\mathscr{D}\left(\mathbf{u}_{n}\right)\left(\mathbf{x}^{\prime}, \mathbf{x}\right)-\gamma \operatorname{Tr}\left[\mathbb{G}_{\rho}^{\mathrm{k}_{2}} \mathbf{u}_{n}(\mathbf{x})\right]\right)\right) d \mathbf{x}^{\prime} d \mathbf{x} \\
& +l_{\Psi}\left[\int_{A} \int_{\Omega^{0}} \rho\left(\mathbf{x}^{\prime}-\mathbf{x}\right) d \mathbf{x}^{\prime} d \mathbf{x}-\int_{B} \int_{\Omega} \rho\left(\mathbf{x}^{\prime}-\mathbf{x}\right) d \mathbf{x}^{\prime} d \mathbf{x}\right]
\end{aligned}
$$

We now increase $A$ to $B$ and $\Omega^{0}$ to $\Omega$ and obtain the inequality (4.9) after noting that

$$
\begin{aligned}
\int_{A} \int_{\Omega^{0}} \rho\left(\mathbf{x}^{\prime}-\mathbf{x}\right) d \mathbf{x}^{\prime} d \mathbf{x}- & \int_{B} \int_{\Omega} \rho\left(\mathbf{x}^{\prime}-\mathbf{x}\right) d \mathbf{x}^{\prime} d \mathbf{x}= \\
& -\int_{B \backslash A} \int_{\Omega} \alpha(\mathbf{x}) \rho\left(\mathbf{x}^{\prime}-\mathbf{x}\right) d \mathbf{x}^{\prime} d \mathbf{x}-\int_{\Omega \backslash \Omega^{0}} \int_{A} \alpha(\mathbf{x}) \rho\left(\mathbf{x}^{\prime}-\mathbf{x}\right) d \mathbf{x}^{\prime} d \mathbf{x}
\end{aligned}
$$

and each of the last terms converges to 0 as $A$ increases to $B$ and $\Omega^{0}$ increases to $\Omega$.

Step 2. Assume that $\alpha(\mathbf{x})=\sum_{i=1}^{k} \alpha_{i} \chi_{B_{i}}(\mathbf{x})$ where $\left\{B_{i}\right\}_{i=1}^{k}$ is a disjoint collection of subsets that cover $\Omega$ and $\alpha_{i} \geq 0$ are real numbers. Applying (4.9) in Step 1 for each $i=1, \cdots, k$ and summing over $i$, we obtain that

$$
\begin{aligned}
\sum_{i=1}^{k} \int_{B_{i}} \int_{\Omega} \alpha_{i} \rho\left(\mathbf{x}^{\prime}-\mathbf{x}\right) \Psi & \left.\left(\mathscr{D}(\mathbf{u})\left(\mathbf{x}^{\prime}, \mathbf{x}\right)-\gamma \operatorname{Tr}\left[\mathbb{G}_{\rho}^{\mathrm{k}_{2}} \mathbf{u}(\mathbf{x})\right]\right)\right) d \mathbf{x}^{\prime} d \mathbf{x} \\
\leq & \left.\sum_{i=1}^{k} \liminf _{n \rightarrow \infty} \int_{B_{i}} \int_{\Omega} \alpha_{i} \rho\left(\mathbf{x}^{\prime}-\mathbf{x}\right) \Psi\left(\mathscr{D}\left(\mathbf{u}_{n}\right)\left(\mathbf{x}^{\prime}, \mathbf{x}\right)-\gamma \operatorname{Tr}\left[\mathbb{G}_{\rho}^{\mathrm{k}_{2}} \mathbf{u}_{n}(\mathbf{x})\right]\right)\right) d \mathbf{x}^{\prime} d \mathbf{x}
\end{aligned}
$$

Using superadditivity of the liminf operation, $\lim \inf a_{n}+\liminf b_{n} \leq \lim \inf \left(a_{n}+b_{n}\right)$, it follows from the above inequality that

$$
\begin{aligned}
\int_{\Omega} \alpha(\mathbf{x}) \int_{\Omega} \rho\left(\mathbf{x}^{\prime}-\mathbf{x}\right) & \left.\Psi\left(\mathscr{D}(\mathbf{u})\left(\mathbf{x}^{\prime}, \mathbf{x}\right)-\gamma \operatorname{Tr}\left[\mathbb{G}_{\rho}^{\mathrm{k}_{2}} \mathbf{u}(\mathbf{x})\right]\right)\right) d \mathbf{x}^{\prime} d \mathbf{x} \\
\leq & \left.\liminf _{n \rightarrow \infty} \int_{\Omega} \int_{\Omega} \alpha(\mathbf{x}) \rho\left(\mathbf{x}^{\prime}-\mathbf{x}\right) \Psi\left(\mathscr{D}\left(\mathbf{u}_{n}\right)\left(\mathbf{x}^{\prime}, \mathbf{x}\right)-\gamma \operatorname{Tr}\left[\mathbb{G}_{\rho}^{\mathrm{k}_{2}} \mathbf{u}_{n}(\mathbf{x})\right]\right)\right) d \mathbf{x}^{\prime} d \mathbf{x}
\end{aligned}
$$

proving the lower semicontinuity inequality that for any nonnegative simple function $\alpha(\mathbf{x})$.

Step 3. Now given any $0 \leq \alpha \in L^{\infty}(\Omega)$, we can find a sequence of simple functions $s_{k}(\mathbf{x})$ such that $s_{k} \rightarrow \alpha$ uniformly in $\Omega$ as $k \rightarrow \infty$ and $0 \leq s_{k}(\mathbf{x}) \leq s_{k+1}(\mathbf{x}) \leq \alpha(\mathbf{x})$, for all $k \in \mathbb{N}$ and all $\mathbf{x} \in \Omega$. We now apply Step 2 to each $s_{k}$ to obtain that

$$
\begin{aligned}
& \left.\int_{\Omega} s_{k}(\mathbf{x}) \int_{\Omega} \rho\left(\mathbf{x}^{\prime}-\mathbf{x}\right) \Psi\left(\mathscr{D}(\mathbf{u})\left(\mathbf{x}^{\prime}, \mathbf{x}\right)-\gamma \operatorname{Tr}\left[\mathbb{G}_{\rho}^{\mathrm{k}_{2}} \mathbf{u}(\mathbf{x})\right]\right)\right) d \mathbf{x}^{\prime} d \mathbf{x} \\
& \left.\left.\leq \liminf _{n \rightarrow \infty} \int_{\Omega} \int_{\Omega} s_{k}(\mathbf{x}) \rho\left(\mathbf{x}^{\prime}-\mathbf{x}\right) \Psi\left(\mathscr{D}\left(\mathbf{u}_{n}\right)\left(\mathbf{x}^{\prime}, \mathbf{x}\right)-\gamma \operatorname{Tr}\left[\mathbb{G}_{\rho}^{\mathrm{k}_{2}} \mathbf{u}_{n}(\mathbf{x})\right]\right)\right)\right) d \mathbf{x}^{\prime} d \mathbf{x} \\
& \left.\leq \liminf _{n \rightarrow \infty} \int_{\Omega} \int_{\Omega} \alpha(\mathbf{x}) \rho\left(\mathbf{x}^{\prime}-\mathbf{x}\right)\left(\Psi\left(\mathscr{D}\left(\mathbf{u}_{n}\right)\left(\mathbf{x}^{\prime}, \mathbf{x}\right)-\gamma \operatorname{Tr}\left[\mathbb{G}_{\rho}^{\mathrm{k}_{2}} \mathbf{u}_{n}(\mathbf{x})\right]\right)\right)-l_{\Psi}\right) d \mathbf{x}^{\prime} d \mathbf{x}+l_{\Psi} \int_{\Omega} \int_{\Omega} s_{k}(\mathbf{x}) \rho\left(\mathbf{x}^{\prime}-\mathbf{x}\right) d \mathbf{x}^{\prime} d \mathbf{x} .
\end{aligned}
$$

Finally we let $k \rightarrow \infty$ and apply Dominated convergence theorem (observe that $\mathbf{u} \in \mathcal{S}_{\rho}^{p}(\Omega)$ ) to obtain the lower semicontinuity estimate for any positive bounded function $\alpha$. 
Limiting functionals. We end the main discussion by stating a result on the variational convergence of sequence of nonlocal functionals studied in the previous subsection. The approach to prove these results is the same as the one used in [28]. In passing we make note of the fact that variational convergence of discrete energies with long range interaction has been studied in $[1,2,10,22]$. $\Gamma$-convergence technique has also been used in [23, 24, 14] to derive local models from peridynamic models. Let us introduce a sequence of radial function $\rho_{n}$ satisfying (3.1), and corresponding operators $E_{\rho_{n}}$. For each $p, q$ and $n$, we simplify the notation $\mathcal{S}_{\rho_{n}}^{p, q}$ and $E_{\rho_{n}}$ by $\mathcal{S}_{n}^{p, q}$ and $E_{n}$ respectively. Let us introduce the following subspace of $L^{p}\left(\Omega ; \mathbb{R}^{d}\right)$ :

$$
\mathcal{X}^{p, q}(\operatorname{div})=\left\{\mathbf{u} \in L^{p}\left(\Omega ; \mathbb{R}^{d}\right): \operatorname{div} \mathbf{u} \in L^{q}(\Omega) ; \text { and } \lim _{n \rightarrow \infty} \operatorname{Tr}\left(\mathbb{G}_{\rho_{n}}^{\mathrm{k}_{2}} \mathbf{u}\right)=\operatorname{div} \mathbf{u}, \text { strongly in } L^{q}\right\} .
$$

For vector fields $\mathbf{u} \in W^{1, p}\left(\Omega ; \mathbb{R}^{d}\right)$ such that $\operatorname{div} \mathbf{u} \in L^{q}(\Omega)$, we introduce the functional

$$
\left.F_{\infty}(\mathbf{u})=\int_{\Omega} \kappa(\mathbf{x}) \Phi\left(\frac{1}{d} \operatorname{div} \mathbf{u}(\mathbf{x})\right) d \mathbf{x}+\int_{\Omega} f_{\mathbb{S}^{d-1}} \alpha(\mathbf{x}) \Psi\left(\mathbf{s} \cdot \nabla \mathbf{u}(\mathbf{x}) \mathbf{s}-\frac{1}{d^{2}} \operatorname{div} \mathbf{u}(\mathbf{x})\right) d \mathcal{H}^{d-1}(\mathbf{s})\right) d \mathbf{x} .
$$

Theorem 4.5. Suppose that $1<p \leq q<\infty$ and assumption A1)-A3) are satisfied. Then we have the following.

1. For each $\mathbf{u} \in W^{1, p}\left(\Omega ; \mathbb{R}^{d}\right)$ such that div $\mathbf{u} \in L^{q}(\Omega)$, and any sequence $\mathbf{u}_{n} \rightarrow \mathbf{u}$ in $L^{p}\left(\Omega ; \mathbb{R}^{d}\right)$, then

$$
F_{\infty}(\mathbf{u}) \leq \liminf _{n \rightarrow \infty} E_{n}\left(\mathbf{u}_{n}\right) .
$$

Moreover, for any $\mathbf{u} \in W^{1, p}\left(\Omega ; \mathbb{R}^{d}\right) \cap \mathcal{X}^{p, q}($ div $)$,

$$
F_{\infty}(\mathbf{u})=\inf \left\{\liminf _{n \rightarrow \infty} E_{n}\left(\mathbf{u}_{n}\right): \mathbf{u}_{n} \rightarrow \mathbf{u} \text { in } L^{p}\left(\Omega ; \mathbb{R}^{d}\right)\right\} .
$$

2. If $\mathbf{u}_{n} \rightarrow \mathbf{u}$ in $L^{p}\left(\Omega ; \mathbb{R}^{d}\right)$, and

$$
\liminf _{n \rightarrow \infty} E_{n}\left(\mathbf{u}_{n}\right)<\infty,
$$

then $\mathbf{u} \in W^{1, p}\left(\Omega ; \mathbb{R}^{d}\right)$ and its divergence div $\mathbf{u} \in L^{q}(\Omega)$.

Proof. The argument follows mostly from standard techniques, in particular mimics the proof of [28, Theorem 1.2]. We only sketch the main steps here. Let us prove Part a). Let $\mathbf{u} \in W^{1, p}\left(\Omega ; \mathbb{R}^{d}\right) \cap\left\{\mathbf{u}: \operatorname{div} \mathbf{u} \in L^{q}(\Omega)\right\}$, and $\mathbf{u}_{n} \rightarrow \mathbf{u}$ in $L^{p}\left(\Omega ; \mathbb{R}^{d}\right)$. Without loss of generality we may assume that $\lim \inf _{n \rightarrow \infty} \bar{E}_{n}\left(\mathbf{u}_{n}\right)<\infty$ and that $\bar{E}_{n}\left(\mathbf{u}_{n}\right)$ is uniformly bounded. From Lemma 4.1 together with the fact that $\mathbf{u}_{n}$ is bounded in $L^{p}\left(\Omega ; \mathbb{R}^{d}\right)$, there exists a positive constant $C$ such that

$$
\left\|\mathbf{u}_{n}\right\|_{L^{p}}^{p}+\left|\mathbf{u}_{n}\right|_{\mathcal{S}_{n}^{p}}^{p}+\left\|\operatorname{Tr}\left[\mathbb{G}_{\rho_{n}}^{\mathrm{k}_{2}}\left(\mathbf{u}_{n}\right)\right]\right\|_{L^{q}}^{q} \leq C E_{n}\left(\mathbf{u}_{n}\right)-C^{2} \leq C
$$

where we used the assumption on the uniform boundedness of $\left\|\mathbf{u}_{n}\right\|_{L^{p}}$ and $E_{n}\left(\mathbf{u}_{n}\right)$. Using Hölder's inequality, there exists a constant $C>0$ such that

$$
\left\|\mathbb{G}_{n}^{\mathrm{k}_{2}} \mathbf{u}_{n}\right\|_{L^{p}} \leq C\left\|\rho_{n}\right\|_{L^{1}}\left|\mathbf{u}_{n}\right|_{\mathcal{S}_{n}^{p}} \leq C .
$$

We can now apply Theorem 3.14 to conclude that $\mathbb{G}_{n}^{\mathrm{k}_{2}} \mathbf{u}_{n}$ weakly converges to $D^{\mathrm{k}_{2}}(\mathbf{u})$ in $L^{p}\left(\Omega ; \mathbb{R}^{d \times d}\right)$. It follows then that $\operatorname{Tr}\left[\mathbb{G}_{n}^{\mathrm{k}_{2}} \mathbf{u}_{n}\right]$ also weakly converges $\operatorname{Tr}\left[D^{\mathrm{k}_{2}}(\mathbf{u})\right]$ in $L^{p}(\Omega)$. As a consequence, since $\operatorname{Tr}\left[\mathbb{G}_{n}^{\mathrm{k}_{2}} \mathbf{u}_{n}\right]$ is bounded in $L^{q}(\Omega)$, there exists a subsequence (not relabeled) such that $\operatorname{Tr}\left[\mathbb{G}_{n}^{\mathrm{k}_{2}} \mathbf{u}_{n}\right]$ weakly converge to $\operatorname{Tr}\left[D^{\mathrm{k}_{2}}(\mathbf{u})\right]$ in $L^{q}(\Omega)$. Note that from our list of examples,

$$
D^{\mathrm{k}_{2}}(\mathbf{u})=\mathrm{C}^{\mathrm{k}_{2}} \nabla \mathbf{u}^{t}=\lambda\left(\nabla \mathbf{u}+\nabla \mathbf{u}^{t}\right)+\lambda \operatorname{div} \mathbf{u} \mathbb{I}
$$

and therefore $\operatorname{Tr}\left[D^{\mathrm{k}_{2}}(\mathbf{u})\right]=(d+2) \lambda$ divu $=\frac{1}{d}$ divu. By the convexity of $\kappa(\mathbf{x}) \Phi(s)$ in the $s$-variable, we see that

$$
\int_{\Omega} \kappa(\mathbf{x}) \Phi\left(\frac{1}{d} \operatorname{div} \mathbf{u}(\mathbf{x})\right) d \mathbf{x} \leq \liminf _{n \rightarrow \infty} \int_{\Omega} \kappa(\mathbf{x}) \Phi\left(\operatorname{Tr}\left[\mathbb{G}_{n}^{\mathrm{k}_{2}} \mathbf{u}_{n}(\mathbf{x})\right]\right) d \mathbf{x} .
$$

The proof that

$$
\begin{aligned}
\int_{\Omega} f_{\mathbb{S}^{d-1}} \alpha & (\mathbf{x}) \Psi\left(\mathbf{s} \cdot \nabla \mathbf{u}(\mathbf{x}) \mathbf{s}-\frac{1}{d^{2}} \operatorname{div} \mathbf{u}(\mathbf{x})\right) d \mathcal{H}^{d-1}(\mathbf{s} d \mathbf{x} \\
& \leq \liminf _{n \rightarrow \infty} \int_{\Omega} \int_{\Omega} \alpha(\mathbf{x}) \rho_{n}\left(\mathbf{x}^{\prime}-\mathbf{x}\right) \Psi\left(\mathscr{D}\left(\mathbf{u}_{n}\right)\left(\mathbf{x}^{\prime}, \mathbf{x}\right)-\frac{1}{d} \operatorname{Tr}\left[\mathbb{G}_{n}^{\mathbf{k}_{2}} \mathbf{u}_{n}(\mathbf{x})\right]\right) d \mathbf{x}^{\prime} d \mathbf{x}
\end{aligned}
$$

uses Lemma 4.2 and follows the same strategy as in the lower semicontinuity proof of Theorem 4.4 (see also a similar argument used in the proof of [28, Corollary 3.7]). We omit the detailed proof. For part 2, as above, 
we may assume without loss of generality that $\left\{E_{n}\left(\mathbf{u}_{n}\right)\right\}$ is a bounded sequence of numbers. Then Lemma 4.1 together with the fact that $\mathbf{u}_{n}$ is bounded in $L^{p}\left(\Omega ; \mathbb{R}^{d}\right)$, implies that there exists a positive constant $C$ such that

$$
\left\|\mathbf{u}_{n}\right\|_{L^{p}}^{p}+\left|\mathbf{u}_{n}\right|_{\mathcal{S}_{n}^{p}}^{p}+\left\|\operatorname{Tr}\left[\mathbb{G}_{\rho_{n}}^{\mathrm{k}_{2}}\left(\mathbf{u}_{n}\right)\right]\right\|_{L^{q}}^{q} \leq C E_{n}\left(\mathbf{u}_{n}\right)-C^{2} \leq C .
$$

As above we argue that $\mathbb{G}_{n}^{\mathrm{k}_{2}}\left(\mathbf{u}_{n}\right)$ is bounded in $L^{p}\left(\Omega ; \mathbb{R}^{d \times d}\right)$ and by Theorem 3.14 any weak limit, and therefore $\mathbf{u} \in \in \mathcal{W}_{\mathrm{k}_{2}}^{p}(\Omega)=W^{1, p}\left(\Omega ; \mathbb{R}^{d}\right)$. Moreover, $\operatorname{Tr}\left[\mathbb{G}_{\rho_{n}}^{\mathrm{k}_{2}}\left(\mathbf{u}_{n}\right)\right]$ weakly converges to $\frac{1}{d} \operatorname{div} \mathbf{u}$ in $L^{p}(\Omega)$. Since $q \geq p$ and $\operatorname{Tr}\left[\mathbb{G}_{\rho_{n}}^{\mathrm{k}_{2}}\left(\mathbf{u}_{n}\right)\right]$ is bounded in $L^{q}(\Omega)$, up to a subsequence its weak limit in $L^{q}$ will coincide with $\frac{1}{d} \operatorname{div} \mathbf{u}$.

Remark 4.6. a) One can view the theorem as a result that presents a lower bound for the $\Gamma$-limit of the sequence of functional $E_{n}$ with respect to the strong $L^{p}$ topology, see [15]. Indeed, from theory of $\Gamma$-convergence up to a subsequence, the sequence of functionals $\bar{E}_{n} \Gamma$-converge in the strong $L^{p}$ topology to a functional $\bar{E}_{\infty}$ where the extended sequence of functionals $\left\{\bar{E}_{n}\right\}_{n \geq 1}$ is given by

$$
\bar{E}_{n}(\mathbf{u})= \begin{cases}E_{n}(\mathbf{u}) & \text { if } \mathbf{u} \in \mathcal{S}_{n}^{p, q}(\Omega) \\ \infty & \text { if } \mathbf{u} \in L^{p}\left(\Omega ; \mathbb{R}^{d}\right) \backslash \mathcal{S}_{n}^{p, p}(\Omega) .\end{cases}
$$

Theorem 4.5 says that the limit functional $\bar{E}_{\infty}: L^{p}\left(\Omega ; \mathbb{R}^{d}\right) \rightarrow \mathbb{R} \cup\{\infty\}$ has the property that

$$
\infty>\bar{E}_{\infty}(\mathbf{u}) \geq F_{\infty}(\mathbf{u}), \quad \text { if } \mathbf{u} \in\left\{W^{1, p}\left(\Omega ; \mathbb{R}^{d}\right): \operatorname{div} \mathbf{u} \in L^{q}(\Omega)\right\}
$$

and $\infty$ otherwise. Moreover, its restriction on $\mathcal{X}^{p, q}($ div $)$ coincides with $F_{\infty}(\mathbf{u})$. Yet, we do not have a closed formula for $\bar{E}_{\infty}(\mathbf{u})$. The convexity of the sequence of functionals suggest that $\bar{E}_{\infty}(\mathbf{u})$ could be precisely $F_{\infty}(\mathbf{u})$. b) When $p=q$ and $\alpha$ and $\kappa$ are positive constants, then its is shown in the recent paper [28] that $\bar{E}_{\infty}(\mathbf{u})=F_{\infty}(\mathbf{u})$ as long as $\mathbf{u} \in W^{1, p}\left(\Omega ; \mathbb{R}^{d}\right)$. Even in this case, Theorem 4.5 can be viewed as an improvement of $[28$, Theorem $1.2]$ to include functionals with 'variable coefficients' $\alpha(\mathbf{x})$ and $\kappa(\mathbf{x})$.

\section{Conclusions}

In this paper, we have established a number of properties of some nonlocal gradient operators using more general tensor-valued kernels. Chief among them is the nonlocal integration by parts formula that is used to characterize standard function spaces. Localization of these nonlocal derivates is also studied. We have presented an application from nonlocal continuum mechanics that uses some of the results proved in the paper. There are obviously many interesting extensions remain to be investigated. For example, applications to the study of nonlocal variational problems with linear growth at infinity and connections to classical problems that used BV/BD spaces remain elusive. As stated in the introduction, we have focused on working with translation invariant kernels and spatial heterogeneities have been introduced only as 'variable coefficients' in the nonlocal variational problems. One may consider further position-dependent kernels to account for more general heterogeneities and effects near or at physical boundary and interfaces. In addition to studies of nonlocal gradient and divergence operators, one may consider nonlocal curl operators like those proposed in [18, 17]. Naturally, it will also be of great interest to consider applications to various nonlinear nonlocal models beyond ones studied here.

\section{ACKNOWLEDGEMENT}

Tadele Mengesha's research is in part supported by NSF grant DMS-1506512. Any opinions, findings, and conclusions or recommendations expressed in this material are those of the author(s) and do not necessarily reflect the views of the National Science Foundation. Qiang Du's research is supported in part by the U.S. NSF grant DMS-1318586, and AFOSR MURI center for material failure prediction through peridynamics.

\section{REFERENCES}

[1] R. Alicandro, M. Cicalese. A general integral representation result for continuum limits of discrete energies with superlinear growth, SIAM J. Math. Anal., 36, 1-37, 2004.

[2] R. Alicandro, M. Focardi and M.S. Gelli. Finite diffrence approximation of energies in fracture mechanics. Ann Scuola Norm. Sup, Pisa Cl. Sci., 29, 671-709, 2000.

[3] L. Ambrosio,N. Fusco and Pallara. Functions of Bounded Variation and Free Discontinuity Problems, Oxford Science Publications, 2000.

[4] F. Andreu-Vaillo, J. M. Mazón, Julio D. Rossi , and J. J. Toledo-Melero, Nonlocal Diffusion Problems, American Mathematical Society. Mathematical Surveys and Monographs, 165, Providence, 2010.

[5] G. Anzellotti and M. Giaquinta. Existence of the displacement field for an elasto-plastic body subject to Hencky's law and Von Mises yield condition, Manuscript Math., 32, 101-136, 1980. 
[6] G. Aubert and P. Kornprobst. Can the nonlocal characterization of Sobolev spaces by Bourgain et al. be useful for solving variational problems? SIAM J. Numer. Anal., 47, 844-860, 2009.

[7] B. Bojarski, L. Ihnatsyeva, J. Kinnunen. How to recognize polynomials in higher order Sobolev spaces, Math. Scand. 112 (2) 161-18, 2013.

[8] R. Borghol, Some properties of Sobolev spaces, Asymptot. Anal. 51 (34) 303-318, 2007.

[9] J. Bourgain, H. Brezis, and P. Mironescu. Another look at Sobolev spaces. In: Menaldi, J.L., et al. (eds.) Optimal control and partial differential equations, pp 439-455 (A volume in honor of A. Benssoussan's 60th birthday). IOS Press, 2001.

[10] A. Braides and M. S. Gelli. Limits of discrete systems with long range interactions, J. Convex Anal., 9, 363-399, 2002.

[11] H. Brezis. How to recognize constant functions. Connections with Sobolev spaces. Russ. Math. Surv., 57, 693-708, 2002.

[12] H. Brezis, Functional Analysis, Sobolev Spaces and Partial Differential Equations, Springer. Chicago, 2010.

[13] J. C. Bellido and C. Mora-Corral. Existence for Nonlocal Variational problems in Peridynamics, SIAM J. Math. Anal., 46, 890-916, 2014.

[14] J. C. Bellido, C. Mora-Corral and P. Pedregal. Hyperelasticity as a $\Gamma$-limit of peridy- namics when the horizon goes to zero. Calculus of Variations and PDEs, 27, 2015, DOI 10.1007/s00526-015-0839-9.

[15] G. Dal Maso. An introduction to $\Gamma$-convergence. Progress in Nonlinear Differential Equations and their Applications, 8. Birkhäuser Boston, Inc., Boston, MA, 1993.

[16] J. Dávila. On an open question about functions of bounded variation. Calc. Var. Partial Differential Equations, 15, 519-527, 2002.

[17] Q. Du, M. Gunzburger, R.B. Lehoucq, and K. Zhou. Analysis of the volume-constrained peridynamic navier equation of linear elasticity. Journal of Elasticity, 113, 193-217, 2013.

[18] Q. Du, M. Gunzburger, R.B. Lehoucq, and K. Zhou. A nonlocal vector calculus, nonlocal volume constrained problems, and nonlocal balance laws. Math. Models Methods Appl. Sci., 23:493-540, 2013.

[19] E. Emmrich and O. Weckner. On the well-posedness of the linear peridynamic model and its convergence towards the Navier equation of linear elasticity. Commun. Math. Sci., 5 851-864, 2007.

[20] L. C. Evans and R. F. Gariepy. Measure Theory and Fine Properties of Functions, CRC Press, Boca Ration, Fl, 1992.

[21] I. Fonseca and G. Leoni. Modern Methods in the Calculus of Variations: L $L^{p}$ Spaces, Springer, 2007.

[22] M. Lewicka and P. Ochoa. On the variational limits of lattice energies on prestrained bodies, Differential Geometry and Continuum Mechanics Editors Gui-Qiang G. Chen, Michael Grinfeld and R.J. Knops, 281-306, 2015.

[23] R. Lipton. Dynamic brittle fracture as a small horizon limit of peridynamics. Journal of Elasticity, 114, 1-30, 2014.

[24] R. Lipton. Cohesive Dynamics and Fracture. Preprint.

[25] T. Mengesha. Nonlocal Korn-type characterization of Sobolev vector fields, Comm. Cont. Math., 14, $1250028,2012$.

[26] T. Mengesha and Q. Du. Nonlocal Constrained Value Problems for a Linear Peridynamic Navier Equation, Journal of Elasticity, 116, 27-51, 2014

[27] T. Mengesha and Q. Du. The bond-based peridynamic system with Dirichlet-type volume constraint, Proceedings of the Royal Society of Edinburgh, 144A, 161-186, 2014.

[28] T. Mengesha and Q. Du. On the variational limit of a class of nonlocal functionals related to peridynamics, Nonlinearity, Nonlinearity, 28:11, 3999-4035, 2015.

[29] T. Mengesha and D. Spector, Localization of nonlocal gradients in various topologies, Calculus of Variations and PDEs, 52, 253-279, 2013.

[30] A. Ponce. An estimate in the spirit of Poincare's inequality, J. Eur. Math. Soc. , 6, 1-15, 2004.

[31] A. Ponce. A new approach to Sobolev spaces and connections to $\Gamma$-convergence. Calc. Var. Partial Differential Equations, 19, 229-255, 2004.

[32] L. Schwartz. Espaces de fonctions differentiables à valerus vectorielles, J. Analyse Math., 4 88-148, 1954.

[33] S.A. Silling. Reformulation of elasticity theory for discontinuities and long-range forces. J. Mech. Phys. Solids, 48, 175-209, 2000.

[34] S.A. Silling. Linearized theory of peridynamic states. J. Elast. 99, 85-111 (2010)

[35] S.A. Silling. Epton, M., Weckner, O., Xu, J., Askari, E. Peridynamic states and constitutive modeling. J. Elast. 88, 151-184, 2007.

[36] R. Temam. Problèmes mathématiques en plasticité, Gauthier-Villars, Paris (1983).

[37] X. Tian and Q. Du. A class of high order nonlocal operators. Preprint.

[38] M.R. Tupek, R. Radovitzky. An extended constitutive correspondence formulation of peridynamics based on nonlinear bond-strain measures, Journal of the Mechanics and Physics of Solids, 65, 82-92, 2014.

[39] M.R. Tupek, J.J. Rimoli, R. Radovitzky. An approach for incorporating classical continuum damage models in statebased peridynamics, Computer Methods in Applied Mechanics and Engineering, 263, 20-26, 2013. 
Tadele Mengesha, Department of Mathematics, University of Tennessee Knoxville, Knoxville, TN 37996

E-mail address: mengesha@utk.edu

Qiang Du, Department of Applied Physics and Applied Mathematics, Columbia University, New York, Ny 10027

E-mail address: qd2125@columbia.edu 